\title{
Synthesis of 3-Acyl-2-arylindole via Palladium-Catalyzed Isocyanide Insertion and Oxypalladation of Alkyne
}

Takeshi Nanjo, Sho Yamamoto, Chihiro Tsukano, Yoshiji Takemoto*

Graduate School of Pharmaceutical Sciences, Kyoto University, Yoshida, Sakyo-ku, Kyoto, 606-8501, Japan

Table of Contents

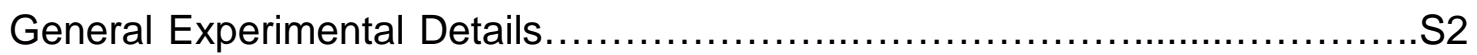

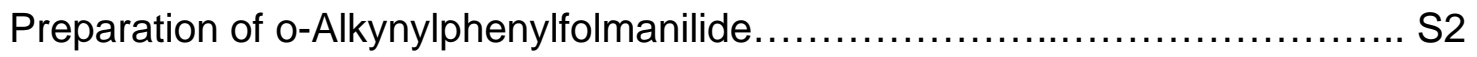

Procedure for the Preparation of o-Alkynylphenylisocyanide $\ldots \ldots \ldots \ldots \ldots \ldots \ldots$ S5

Procedure for the Synthesis of $3-A c y l-2$-arylindoles.............................. 55

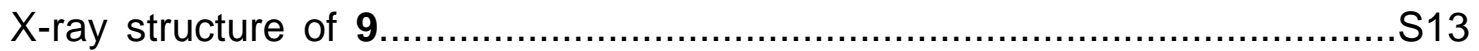

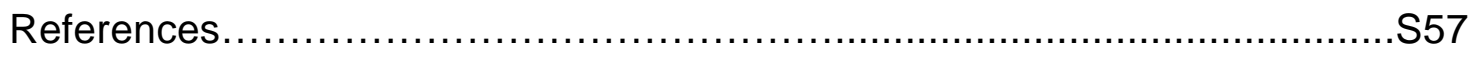

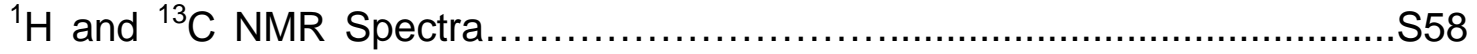




\section{General Experimental Details}

Unless otherwise noted, all reactions were performed under argon. $\mathrm{Pd}(\mathrm{OAc})_{2}$ and $\mathrm{Ad}_{2} \mathrm{P}^{n} \mathrm{Bu}$ were purchased from Sigma Aldrich. DMF and $\mathrm{Cs}_{2} \mathrm{CO}_{3}$ were purchased from Wako Pure Chemical Industries, Ltd.. Unless otherwise noted, all other reagents were purchased from commercial suppliers and used as received.

Analytical thin-layer chromatography was performed with Merck Silica gel 60. Silica gel column chromatography was performed with Kanto silica gel 60 (particle size, 63-210 $\mu \mathrm{m}$ ) or Fuji Silysia BW-300. Column chromatography using Aluminum oxide was performed with SIGMAALDRICH Aluminum oxide. All melting points (m.p.) were determined on YANAGIMOTO micro melting point apparatus and BÜCHI Melting Point M-565. Proton nuclear magnetic resonance $\left({ }^{1} \mathrm{H}\right.$ NMR) spectra were recorded on a JEOL JNM-LA 500 at $500 \mathrm{MHz}$. Chemical shifts are reported relative to $\mathrm{Me}_{4} \mathrm{Si}(\delta 0.00)$. Multiplicity is indicated by one or more of the following: $\mathrm{s}$ (singlet); d (doublet); t (triplet); q (quartet); sep (septet); m (multiplet); br (broad). Carbon nuclear magnetic resonance $\left({ }^{13} \mathrm{C}\right.$ NMR) spectra were recorded on a JEOL JNM-LA 500 at 126 $\mathrm{MHz}$. Chemical shifts are reported relative to $\mathrm{CDCl}_{3}(\delta 77.0), \mathrm{DMF}-d_{7}(\delta 162.7)$, and DMSO- $d_{6}$ ( $\delta 39.5)$. Infrared spectra were recorded on a FT/IR-4100 (JASCO). Low and high resolution mass spectra were recorded on JEOL JMS-HX/HX 110A

\section{Preparation of o-Alkynylphenylfolmanilide}

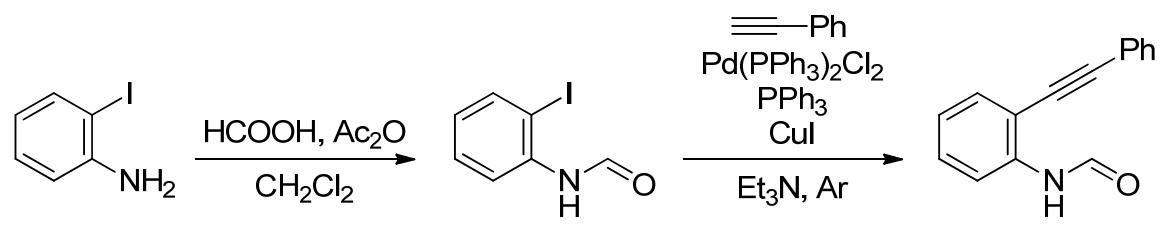

N-(2-(Phenylethynyl)phenyl)formamide (S1): To formic acid (2.41 mL, $63.9 \mathrm{mmol})$ was added acetic anhydride $(2.16 \mathrm{~mL}, 22.8 \mathrm{mmol})$ and the mixure was stirred at room temperature. After stirring for $10 \mathrm{~min}$, to the mixture was added the solution of $o$-iodoaniline $(3.99 \mathrm{~g}, 18.3$ $\mathrm{mmol})$ in $\mathrm{CH}_{2} \mathrm{Cl}_{2}(60 \mathrm{~mL})$ and the mixture was stirred at room temperature. After stirring for $2 \mathrm{~h}$, the mixture was concentrated under reduced pressure to give 0 -iodoformanilide ${ }^{2}(4.42 \mathrm{~g}, 98 \%)$ as a white solid.

To the solution of the formanilide (1.01 g, $4.09 \mathrm{mmol}$ ), ethynyl benzene (499 $\mathrm{mg}, 4.89 \mathrm{mmol}$ ) and $\mathrm{Et}_{3} \mathrm{~N}(15 \mathrm{~mL})$ were added $\mathrm{Pd}\left(\mathrm{PPh}_{3}\right)_{2} \mathrm{Cl}_{2}(140 \mathrm{mg}, 0.20 \mathrm{mmol})$ and $\mathrm{Cul}(38.3 \mathrm{mg}, 0.20$ $\mathrm{mmol}$ ) and stirred at room temperature. After stirring for $24 \mathrm{~h}$, the reaction mixture was diluted 
with $\mathrm{Et}_{2} \mathrm{O}$ and the precipitate was removed by filtration through Celite. The filtrate was acidified with saturated aqueous solution of $\mathrm{NH}_{4} \mathrm{Cl}$ and extracted with $\mathrm{Et}_{2} \mathrm{O}$. The combined extracts were washed with brine, dried over $\mathrm{Na}_{2} \mathrm{SO}_{4}$ and concentrated under reduced pressure. The obtained residue was purified by silica gel column chromatography (hexane/EtOAc $=90 / 10$ ) to give $14(0.614 \mathrm{~g}, 68 \%)$ as a white solid: m.p. $96.8-97.9^{\circ} \mathrm{C} ;{ }^{1} \mathrm{H}$ NMR $\left(500 \mathrm{MHz}, \mathrm{CDCl}_{3}\right) \delta 8.83(\mathrm{~d}$, $0.35 \mathrm{H}, \mathrm{J}=11.5 \mathrm{~Hz}), 8.51(\mathrm{~s}, 0.65 \mathrm{H}), 8.44(\mathrm{~d}, 0.65 \mathrm{H}, \mathrm{J}=8.3 \mathrm{~Hz}), 7.99(\mathrm{brs}, 1 \mathrm{H}), 7.56-7.51(\mathrm{~m}$, $3 \mathrm{H}), 7.31-7.40(\mathrm{~m}, 4 \mathrm{H}), 7.25-7.23(\mathrm{~m}, 0.35 \mathrm{H}), 7.17-7.09(\mathrm{~m}, 1 \mathrm{H}) .{ }^{13} \mathrm{C} \mathrm{NMR}\left(126 \mathrm{MHz}, \mathrm{CDCl}_{3}\right)$ $\delta$ 161.3, 159.0, 137.8, 137.6, 133.0, 131.9, 131.7, 131.6, 129.7, 129.7, 129.0, 128.5, 128.5, 124.5, 124.0, 122.2, 122.1, 120.0, 116.2, 113.5, 112.1, 96.5, 83.9, 83.7; IR (ATR) 3140, 1687, $1269 \mathrm{~cm}^{-1} ; \mathrm{MS}(\mathrm{FAB}+) \mathrm{m} / \mathrm{z}=221\left([\mathrm{M}]^{+}\right)$; HRMS (FAB+) Calcd for $\mathrm{C}_{15} \mathrm{H}_{12} \mathrm{NO}[\mathrm{M}+\mathrm{H}]^{+}: 222.0919$; found:222.0916. The peaks of ${ }^{1} \mathrm{H}$ NMR and ${ }^{13} \mathrm{C}$ NMR were observed as the mixture of amide rotamers. The spectroscopic data were in agreement with those reported in the literature. ${ }^{1}$

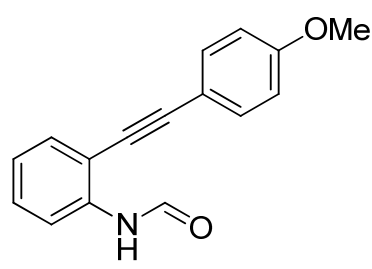

N-(2-((4-Methoxyphenyl)ethynyl)phenyl)formamide (S2): The reaction was performed according to the procedure for S1. A white solid: m.p. $61.0-62.3^{\circ} \mathrm{C} ;{ }^{1} \mathrm{H} \mathrm{NMR}\left(500 \mathrm{MHz}, \mathrm{CDCl}_{3}\right) \delta$ $8.85(\mathrm{~d}, 0.35 \mathrm{H}, J=11.2 \mathrm{~Hz}), 8.52(\mathrm{~s}, 0.65 \mathrm{H}), 8.44(\mathrm{~d}, 0.65 \mathrm{H}, \mathrm{J}=8.3 \mathrm{~Hz}), 7.95(\mathrm{brs}, 1 \mathrm{H}), 7.55-$ $7.46(\mathrm{~m}, 3 \mathrm{H}), 7.37-7.29(\mathrm{~m}, 1 \mathrm{H}), 7.26-7.24(\mathrm{~m}, 0.35 \mathrm{H}), 7.18-7.07(\mathrm{~m}, 1 \mathrm{H}), 6.91(\mathrm{t}, 2 \mathrm{H}, \mathrm{J}=7.9$ $\mathrm{Hz}), 3.85$ (s, 3H); ${ }^{13} \mathrm{C}$ NMR $\left(126 \mathrm{MHz}, \mathrm{CDCl}_{3}\right) \delta 161.3,160.1,158.9,137.7,137.4,133.1$, 133.1, 132.7, 131.7, 129.4, 129.3, 124.4, 123.9, 119.9, 116.0, 114.2, 114.1, 114.1, 114.1, 113.8, 112.4, 96.7, 96.6, 82.6, 82.4, 55.3; IR (ATR) 3273, 1658, 1507, $1246 \mathrm{~cm}^{-1}$; MS (FAB+) $\mathrm{m} / \mathrm{z}=251\left([\mathrm{M}]^{+}\right) ; \mathrm{HRMS}(\mathrm{FAB}+)$ Calcd for $\mathrm{C}_{16} \mathrm{H}_{13} \mathrm{NO}_{2}[\mathrm{M}]^{+}: 251.0946$; found:251.0941. The peaks of ${ }^{1} \mathrm{H}$ NMR and ${ }^{13} \mathrm{C}$ NMR were observed as the mixture of amide rotamers.
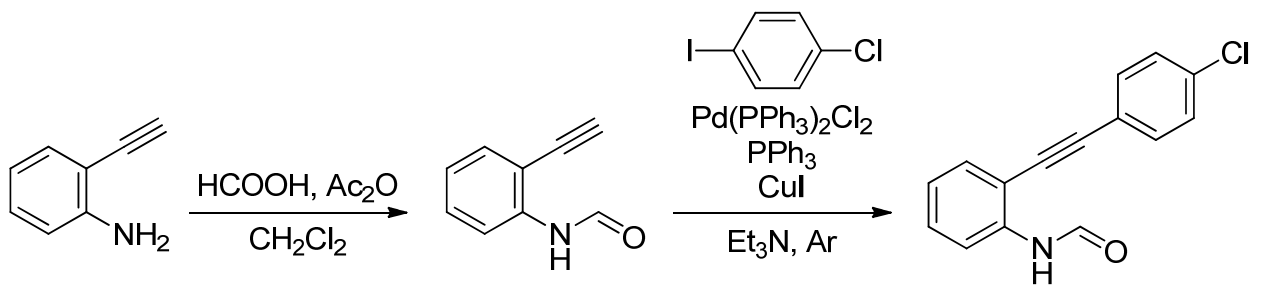
N-(2-((4-Chlorophenyl)ethynyl)phenyl)formamide (S3): To formic acid (1.21 mL, 32.0 $\mathrm{mmol})$ was added acetic anhydride $(1.08 \mathrm{~mL}, 11.4 \mathrm{mmol})$ and the mixture was stirred at room temperature. After stirring for $10 \mathrm{~min}$, to the mixture was added the solution of $o$-ethynylaniline $(1.07 \mathrm{~g}, 9.13 \mathrm{mmol})$ in $\mathrm{CH}_{2} \mathrm{Cl}_{2}(30 \mathrm{~mL})$ and the mixture was stirred at room temperature. After stirring for $1 \mathrm{~h}$, the mixture was concentrated under reduced pressure to give $o$ ethynylformanilide (1.32 $\mathrm{g}, 100 \%)$ as a white solid.

To the solution of the formanilide $(1.32 \mathrm{~g}, 9.13 \mathrm{mmol}), 1$-chloro-4-iodobenzene $(1.81 \mathrm{~g}, 7.61$ $\mathrm{mmol})$ and $\mathrm{Et}_{3} \mathrm{~N}(28 \mathrm{~mL})$ under $\mathrm{Ar}$ atmosphere were added $\mathrm{Pd}\left(\mathrm{PPh}_{3}\right)_{2} \mathrm{Cl}_{2}(260.5 \mathrm{mg}, 0.38$ $\mathrm{mmol}$ ) and Cul (76.5 mg, $0.38 \mathrm{mmol}$ ) and stirred at room temperature. After stirring for $12 \mathrm{~h}$, the reaction mixture was diluted with $\mathrm{CHCl}_{3}$ and the precipitate was removed by filtration through Celite. The filtrate was acidified with saturated aqueous solution of $\mathrm{NH}_{4} \mathrm{Cl}$ and extracted with $\mathrm{CHCl}_{3}$. The combined extracts were washed with brine, dried over $\mathrm{Na}_{2} \mathrm{SO}_{4}$ and concentrated under reduced pressure. The obtained residue was purified by silica gel column chromatography $\left(100 \% \mathrm{CH}_{2} \mathrm{Cl}_{2}\right)$ to give $\mathrm{S} 3(1.10 \mathrm{~g}, 47 \%)$ as a white solid. Mixture of rotamers in a ratio of 0.65:0.35; m.p. $143.7-144.7^{\circ} \mathrm{C} ;{ }^{1} \mathrm{H}$ NMR $\left(500 \mathrm{MHz}, \mathrm{CDCl}_{3}\right) \delta 8.84(\mathrm{~d}, 0.35 \mathrm{H}, \mathrm{J}=$ $11.5 \mathrm{~Hz}), 8.52(\mathrm{~s}, 0.65 \mathrm{H}), 8.44(\mathrm{~d}, 0.65 \mathrm{H}, \mathrm{J}=8.3 \mathrm{~Hz}), 7.95-7.82(\mathrm{~m}, 1 \mathrm{H}), 7.56-7.10(\mathrm{~m}, 7.35 \mathrm{H})$;

${ }^{13} \mathrm{C}$ NMR $\left(126 \mathrm{MHz}, \mathrm{CDCl}_{3}\right) \delta 161.3,158.8,137.8,137.6,135.1,133.0,132.8,132.8,132.0$, $130.0,129.9,128.9,128.9,124.6,124.0,120.7,120.5,120.1,116.3,113.3,111.7,95.3,95.3$, 84.9, 84.6; IR (ATR) 3263, 1654, $1085 \mathrm{~cm}^{-1}$; MS (FAB+) $\mathrm{m} / \mathrm{z}=255\left([\mathrm{M}]^{+}\right)$; HRMS (FAB+) Calcd for $\mathrm{C}_{15} \mathrm{H}_{10} \mathrm{CINO}[\mathrm{M}]^{+}: 255.0451$; found:255.0453. The peaks of ${ }^{1} \mathrm{H}$ NMR and ${ }^{13} \mathrm{C}$ NMR were observed as the mixture of amide rotamers.

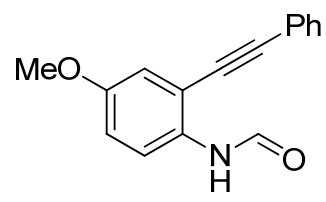

N-(4-Methoxy-2-(phenylethynyl)phenyl)formamide (S4): The reaction was performed according to the procedure for S1. A white solid: m.p. $121.0-122.0^{\circ} \mathrm{C} ;{ }^{1} \mathrm{H} \mathrm{NMR}\left(500 \mathrm{MHz}, \mathrm{CDCl}_{3}\right)$ $\delta 8.63(\mathrm{~d}, 0.35 \mathrm{H}, \mathrm{J}=11.5 \mathrm{~Hz}), 8.43(\mathrm{~s}, 0.65 \mathrm{H}), 8.30(\mathrm{~d}, 0.65 \mathrm{H}, \mathrm{J}=9.2 \mathrm{~Hz}), 7.89-7.83(\mathrm{~m}, 1 \mathrm{H})$, 7.55-7.52 (m, 2H), 7.40-7.33 (m, 3H), 7.12-7.00 (m, 1.35H), 6.92-6.86 (m, 1H), $3.79(\mathrm{~m}, 3 \mathrm{H})$; ${ }^{13} \mathrm{C}$ NMR $\left(126 \mathrm{MHz}, \mathrm{CDCl}_{3}\right) \delta 161.8,158.5,156.7,155.7,131.7,131.7,131.5,130.9,129.1$, 129.1, 128.6, 128.5, 122.1, 122.0, 121.6, 119.2, 117.0, 116.3, 115.9, 115.6, 113.3, 96.3, 96.2, 83.9, 55.7, 55.6; IR (ATR) 3265, 1658, 1532, $1219 \mathrm{~cm}^{-1}$; MS (FAB+) $\mathrm{m} / \mathrm{z}=251\left([\mathrm{M}]^{+}\right)$; HRMS 
$(\mathrm{FAB}+)$ Calcd for $\mathrm{C}_{16} \mathrm{H}_{13} \mathrm{NO}_{2}[\mathrm{M}]^{+}: 251.0946$; found:251.0947. The peaks of ${ }^{1} \mathrm{H}$ NMR and ${ }^{13} \mathrm{C}$ NMR were observed as the mixture of amide rotamers.

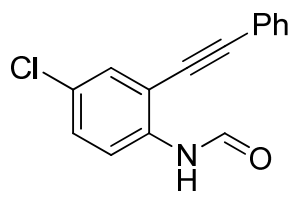

N-(4-Chloro-2-(phenylethynyl)phenyl)formamide (S5): The reaction was performed according to the procedure for S1. A white solid: m.p. $118.8-119.5^{\circ} \mathrm{C} ;{ }^{1} \mathrm{H}$ NMR $\left(500 \mathrm{MHz}, \mathrm{CDCl}_{3}\right) \delta$ $8.78(\mathrm{~d}, 0.25 \mathrm{H}, \mathrm{J}=11.2 \mathrm{~Hz}), 8.49(\mathrm{~s}, 0.75 \mathrm{H}), 8.40(\mathrm{~d}, 0.75 \mathrm{H}, \mathrm{J}=8.9 \mathrm{~Hz}), 7.99-7.88(\mathrm{~m}, 1 \mathrm{H})$, 7.58-7.13 (m, 7.25H); ${ }^{13} \mathrm{C}$ NMR (126 MHz, $\left.\mathrm{CDCl}_{3}\right) \delta 161.0,158.8,136.4,136.2,132.5,131.7$, $131.7,131.4,129.8,129.7,129.4,128.9,128.6,128.6,121.7,121.5,121.1,117.3,115.1$, 113.6, 97.6, 82.6; IR (ATR) 3258, 1643, 1519, $1403 \mathrm{~cm}^{-1}$; MS (FAB+) $\mathrm{m} / \mathrm{z}=255\left([\mathrm{M}]^{+}\right)$; HRMS $(\mathrm{FAB}+)$ Calcd for $\mathrm{C}_{15} \mathrm{H}_{10} \mathrm{CINO}[\mathrm{M}]^{+}: 255.0451$; found:255.0448. The peaks of ${ }^{1} \mathrm{H} N M R$ and ${ }^{13} \mathrm{C}$ NMR were observed as the mixture of amide rotamers.

\section{Procedure for the Preparation of o-Alkynylphenylisocyanide ${ }^{3}$}<smiles>CCOC(=O)N(CC)C(=O)OCCNc1ccccc1C#Cc1ccccc1</smiles>

1-Isocyano-2-(phenylethynyl)benzene (3a): To the solution of formanilide ( $44.3 \mathrm{mg}, 0.200$ $\mathrm{mmol})$ and $\mathrm{Et}_{3} \mathrm{~N}(0.139 \mathrm{~mL}, 1.00 \mathrm{mmol})$ in THF $(2 \mathrm{~mL})$ were added $\mathrm{POCl}_{3}(0.028 \mathrm{~mL}, 0.300$ $\mathrm{mmol})$ at $0{ }^{\circ} \mathrm{C}$ and stirred at the same temperature. After stirring for $1 \mathrm{~h}$, the reaction was quenched with saturated aqueous solution of $\mathrm{NaHCO}_{3}$ and extracted with $\mathrm{Et}_{2} \mathrm{O}$. The combined extracts were washed with brine, dried over $\mathrm{Na}_{2} \mathrm{SO}_{4}$, added toluene $(5 \mathrm{ml})$, and concentrated under reduced pressure to $3 \mathrm{~mL}$. The obtained solution was filtered through alumina column chromatography (hexane/EtOAc $=90 / 10)$. To the obtained solution was added DMF $(2 \mathrm{ml})$ and concentrated under reduced pressure to $2 \mathrm{~mL}$. The solution was used for the synthesis of 3acyl-2-arylindoles.

\section{Procedure for the Synthesis of 3-Acyl-2-arylindoles}




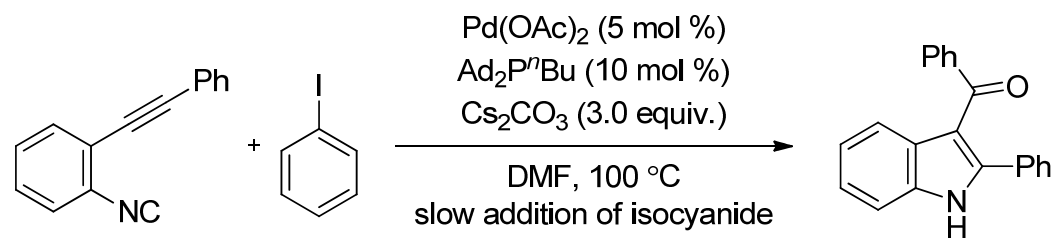

Phenyl(2-phenyl-1H-indol-3-yl)methanone (1a): To a stirred solution iodobenzene (20.4 mg, $0.100 \mathrm{mmol})$ in $\mathrm{DMF}(1 \mathrm{~mL})$ were added $\mathrm{Pd}(\mathrm{OAc})_{2}(1.1 \mathrm{mg}, 0.0049 \mathrm{mmol}), \mathrm{Ad}_{2} \mathrm{P}^{n} \mathrm{Bu}(3.6 \mathrm{mg}$, $0.0101 \mathrm{mmol})$ and $\mathrm{Cs}_{2} \mathrm{CO}_{3}(97.7 \mathrm{mg}, 0.300 \mathrm{mmol})$ at room temperature and the reaction mixture was heated to $100^{\circ} \mathrm{C}$. After stirring for $10 \mathrm{~min}$ freshly, prepared 1-isocyano-2(phenylethynyl)benzene in $\operatorname{DMF}(2 \mathrm{~mL})$ was added for $3 \mathrm{~h}$ and stirred at $100{ }^{\circ} \mathrm{C}$. After stirring for $1 \mathrm{~h}$, the reaction mixture was neutralized by saturated aqueous solution of $\mathrm{NH}_{4} \mathrm{Cl}$ and extracted with EtOAc. The combined extracts were washed with brine, dried over $\mathrm{Na}_{2} \mathrm{SO}_{4}$ and concentrated under reduced pressure. The obtained residue was purified by silica gel column chromatography (hexane/EtOAc $=9 / 1)$ to give $1 \mathrm{a}(23.2 \mathrm{mg}, 78 \%)$ as a white solid; m.p. 224.8$225.8^{\circ} \mathrm{C} ;{ }^{1} \mathrm{H}$ NMR $\left(500 \mathrm{MHz}, \mathrm{CDCl}_{3}\right) \delta 8.65$ (brs, 1H), $7.94(\mathrm{~d}, 1 \mathrm{H}, \mathrm{J}=8.0 \mathrm{~Hz}), 7.65(\mathrm{~d}, 2 \mathrm{H}, \mathrm{J}=$ $7.0 \mathrm{~Hz}), 7.45(\mathrm{~d}, 1 \mathrm{H}, \mathrm{J}=8.0 \mathrm{~Hz}), 7.37-7.16(\mathrm{~m}, 10 \mathrm{H}) ;{ }^{13} \mathrm{C}$ NMR $\left(126 \mathrm{MHz}, \mathrm{DMF}-\mathrm{d}_{7}\right) \delta 193.1$, $144.7,141.0,137.0,132.8,132.0,130.3,130.0,129.4,129.1,128.8,128.5,123.6,122.0$, 121.5, 113.4, 112.5; IR (ATR) 3053, 1563, 1448, 1421, $1216 \mathrm{~cm}^{-1} ; \mathrm{MS}(\mathrm{FAB}+) \mathrm{m} / \mathrm{z}=298$ $\left([\mathrm{M}+\mathrm{H}]^{+}\right)$; HRMS $(\mathrm{FAB}+)$ Calcd for $\mathrm{C}_{21} \mathrm{H}_{16} \mathrm{NO}[\mathrm{M}+\mathrm{H}]^{+}:$298.1232; found: 298.1227. The spectroscopic data were in agreement with those reported in the literature. ${ }^{4}$

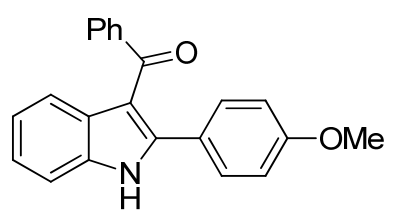

(2-(4-Methoxyphenyl)-1H-indol-3-yl)(phenyl)methanone (1b): The reaction was performed in DMF at $100{ }^{\circ} \mathrm{C}$ according to the procedure for $\mathbf{1 a}$, and gave $\mathbf{1 b}$ in $83 \%$ yield as a white solid: m.p. 210.0-211.0 ${ }^{\circ} \mathrm{C} ;{ }^{1} \mathrm{H}$ NMR (500 MHz, DMSO- $\left.d_{6}\right) \delta 12.13$ (brs, $\left.1 \mathrm{H}\right), 7.75(\mathrm{~d}, 1 \mathrm{H}, \mathrm{J}=8.0 \mathrm{~Hz}$ ), 7.57-7.52 (m, 3H), $7.43(\mathrm{t}, 1 \mathrm{H}, \mathrm{J}=7.3 \mathrm{~Hz}), 7.37$ (d, 2H, J = 8.5 Hz), 7.29-7.25 (m, 3H), 7.18 (t, $1 \mathrm{H}, \mathrm{J}=7.6 \mathrm{~Hz}), 6.86(\mathrm{~d}, 2 \mathrm{H}, \mathrm{J}=8.6 \mathrm{~Hz}), 3.76(\mathrm{~s}, 3 \mathrm{H}) ;{ }^{13} \mathrm{C}$ NMR $\left(126 \mathrm{MHz}, \mathrm{DMSO}-d_{6}\right) \delta 192.1$, $159.4,144.2,139.9,135.7,131.2,130.9,129.0,128.3,127.8,123.8,122.6,121.3,120.4$, 113.5, 111.7, 111.5, 55.2; IR (ATR) 3183, 1591, 1570, 1431, $1247 \mathrm{~cm}^{-1}$; MS (FAB+) $\mathrm{m} / \mathrm{z}=328$ $\left([\mathrm{M}+\mathrm{H}]^{+}\right)$; HRMS $(\mathrm{FAB}+)$ Calcd for $\mathrm{C}_{22} \mathrm{H}_{18} \mathrm{NO}_{2}[\mathrm{M}+\mathrm{H}]^{+}:$328.1338; found: 328.1341. 


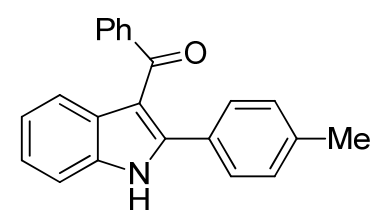

Phenyl(2-(p-tolyl)-1H-indol-3-yl)methanone (1c): The reaction was performed in DMF at $100{ }^{\circ} \mathrm{C}$ according to the procedure for $\mathbf{1 a}$, and gave $1 \mathrm{c}$ in $73 \%$ yield as a brown solid: m.p. 188.0-189.0 ${ }^{\circ}$; ${ }^{1} \mathrm{H}$ NMR (500 MHz, DMF- $\left.d_{7}\right) \delta 12.11$ (brs, $\left.1 \mathrm{H}\right), 7.76(\mathrm{~d}, 1 \mathrm{H}, \mathrm{J}=8.0 \mathrm{~Hz}), 7.65$ (d, $2 \mathrm{H}, \mathrm{J}=7.4 \mathrm{~Hz}), 7.57(\mathrm{~d}, 1 \mathrm{H} \mathrm{J}=8.0 \mathrm{~Hz}), 7.43-7.40(\mathrm{~m}, 3 \mathrm{H}), 7.29-7.24(\mathrm{~m}, 3 \mathrm{H}), 7.16\left(\mathrm{dd}, 1 \mathrm{H}, \mathrm{J}_{1}\right.$ $\left.=J_{2}=7.4 \mathrm{~Hz}\right), 7.10(\mathrm{~d}, 2 \mathrm{H} \mathrm{J}=8.0 \mathrm{~Hz}), 2.26(\mathrm{~s}, 3 \mathrm{H}) ;{ }^{13} \mathrm{C}$ NMR $\left(126 \mathrm{MHz}, \mathrm{DMF}-\mathrm{d}_{7}\right) \delta 193.1$, $144.8,141.0,139.0,137.0,132.0,130.2,130.0,129.9,129.4,129.4,128.5,123.4,121.9$, 121.4, 113.1, 112.4, 21.0; IR (ATR) 3144, 1567, 1428, $1210 \mathrm{~cm}^{-1}$; MS (FAB+) $\mathrm{m} / \mathrm{z}=312$ $\left([\mathrm{M}+\mathrm{H}]^{+}\right)$; HRMS (FAB+) Calcd for $\mathrm{C}_{22} \mathrm{H}_{18} \mathrm{NO}[\mathrm{M}+\mathrm{H}]^{+}: 312.1388$; found:312.1387.

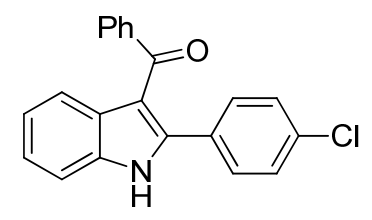

(2-(4-Chlorophenyl)-1H-indol-3-yl)(phenyl)methanone (1d): The reaction was performed in DMF at $100{ }^{\circ} \mathrm{C}$ according to the procedure for $\mathbf{1 a}$, and gave $\mathbf{1 d}$ in $93 \%$ yield as a white solid: m.p. 221.0-222.0 ${ }^{\circ} \mathrm{C} ;{ }^{1} \mathrm{H}$ NMR (500 MHz, DMF- $\left.d_{7}\right) \delta 12.13$ (brs, $\left.1 \mathrm{H}\right), 7.65$ (d, $1 \mathrm{H}, \mathrm{J}=8.0 \mathrm{~Hz}$ ), 7.49-7.44 (m, 3H), 7.39 (d, 2H, J = 8.3 Hz), 7.29 (dd, $\left.1 \mathrm{H}, \mathrm{J}_{1}=\mathrm{J}_{2}=7.4 \mathrm{~Hz}\right), 7.20(\mathrm{~d}, 2 \mathrm{H}, \mathrm{J}=8.5$ $\mathrm{Hz}), 7.16-7.13(\mathrm{~m}, 3 \mathrm{H}), 7.05\left(\mathrm{dd}, 1 \mathrm{H}, \mathrm{J}_{1}=\mathrm{J}_{2}=7.4 \mathrm{~Hz}\right) ;{ }^{13} \mathrm{C} \mathrm{NMR}\left(126 \mathrm{MHz}, \mathrm{DMF}-d_{7}\right) \delta 192.9$, 143.4, 141.0, 137.0, 134.4, 132.1, 132.0, 131.6, 130.0, 129.2, 128.9, 128.6, 123.8, 122.1, 121.5, 113.7, 112.6; IR (ATR) 3134, 1598, 1567, 1416, $1208 \mathrm{~cm}^{-1}$; MS (FAB+) m/z = 332 $\left([\mathrm{M}+\mathrm{H}]^{+}\right)$; HRMS $(\mathrm{FAB}+)$ Calcd for $\mathrm{C}_{21} \mathrm{H}_{15} \mathrm{CINO}[\mathrm{M}+\mathrm{H}]^{+}: 332.0842$; found:332.0841.

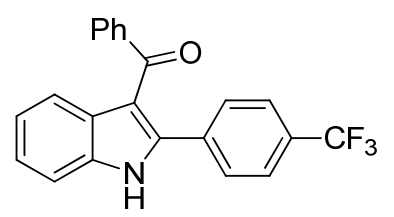

Phenyl(2-(4-(trifluoromethyl)phenyl)-1H-indol-3-yl)methanone (1e): The reaction was performed in DMF at $100{ }^{\circ} \mathrm{C}$ according to the procedure for $1 \mathbf{a}$, and gave $1 \mathbf{e}$ in $64 \%$ yield as a white solid; m.p. 224.0-225.0 ${ }^{\circ} \mathrm{C} ;{ }^{1} \mathrm{H}$ NMR (500 MHz, DMF- $\left.d_{7}\right) \delta 12.40$ (brs, $\left.1 \mathrm{H}\right), 7.79-7.74(\mathrm{~m}$, $3 \mathrm{H}), 7.67-7.61(\mathrm{~m}, 5 \mathrm{H}), 7.43\left(\mathrm{dd}, 1 \mathrm{H}, \mathrm{J}_{1}=\mathrm{J}_{2}=7.4 \mathrm{~Hz}\right), 7.33-7.27(\mathrm{~m}, 3 \mathrm{H}), 7.21\left(\mathrm{dd}, 1 \mathrm{H}, \mathrm{J}_{1}=J_{2}\right.$ 
$=7.4 \mathrm{~Hz}) ;{ }^{13} \mathrm{C}$ NMR $\left(126 \mathrm{MHz}\right.$, DMF- $\left.d_{7}\right) \delta 192.9,142.8,140.9,137.2,136.8,132.2,131.1$, 130.0, 129.7 (q, J = 31.4 Hz), 129.1, 128.6, 125.6 (q, J = 3.6 Hz) 125.1 (q, J = 271.1 Hz) 124.1, 122.3, 121.7, 114.5, 112.7; IR (ATR) 3077, 1595, 1563, 1427, $1320 \mathrm{~cm}^{-1}$; MS (FAB+) $\mathrm{m} / \mathrm{z}=$ $366\left([\mathrm{M}+\mathrm{H}]^{+}\right)$; HRMS (FAB+) Calcd for $\mathrm{C}_{22} \mathrm{H}_{15} \mathrm{~F}_{3} \mathrm{NO}[\mathrm{M}+\mathrm{H}]^{+}: 366.1106$; found:366.1102.

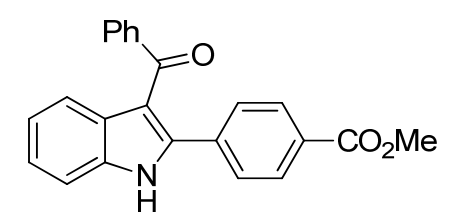

Methyl 4-(3-benzoyl-1H-indol-2-yl)benzoate (1f): The reaction was performed in DMF at 100 ${ }^{\circ} \mathrm{C}$ according to the procedure for $1 \mathbf{a}$, and gave $1 \mathrm{f}$ in $48 \%$ yield as a yellow solid; m.p. 214.0$215.0^{\circ} \mathrm{C} ;{ }^{1} \mathrm{H}$ NMR $\left(500 \mathrm{MHz}\right.$, DMF- $\left.d_{7}\right) \delta 12.24$ (brs, $\left.1 \mathrm{H}\right), 7.73(\mathrm{~d}, 2 \mathrm{H}, \mathrm{J}=8.3 \mathrm{~Hz}), 7.67(\mathrm{~d}, 1 \mathrm{H}$, $\mathrm{J}=8.0 \mathrm{~Hz}$ ), 7.53-7.47 (m,5H), 7.27 (dd, $\left.1 \mathrm{H}, \mathrm{J}_{1}=\mathrm{J}_{2}=7.4 \mathrm{~Hz}\right), 7.18-7.12(\mathrm{~m}, 3 \mathrm{H}), 7.07(\mathrm{dd}, 1 \mathrm{H}$, $\left.J_{1}=J_{2}=7.4 \mathrm{~Hz}\right), 3.74(\mathrm{~s}, 3 \mathrm{H}) ;{ }^{13} \mathrm{C} \mathrm{NMR}\left(126 \mathrm{MHz}, \mathrm{DMF}-d_{7}\right) \delta 193.0,166.7,143.0,140.9$, 137.3, 132.3, 130.5, 130.1, 130.1, 129.6, 129.3, 128.6, 124.0, 122.2, 121.6, 114.3, 112.7, 52.5 (One carbon peak was overlapping); IR (ATR) 3178, 1724, 1611, 1571, 1432, 1274, $1104 \mathrm{~cm}^{-}$ 1; MS (FAB+) m/z = $356\left([M+H]^{+}\right)$; HRMS (FAB+) Calcd for $\mathrm{C}_{23} \mathrm{H}_{18} \mathrm{NO}_{3}[\mathrm{M}+\mathrm{H}]^{+}: 356.1287$; found:356.1288.

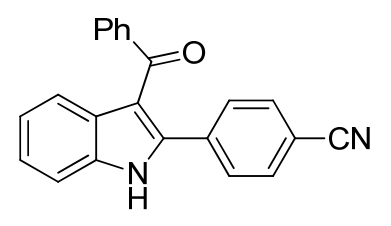

4-(3-Benzoyl-1H-indol-2-yl)benzonitrile (1g): The reaction was performed in DMF at $100{ }^{\circ} \mathrm{C}$ according to the procedure for $1 \mathbf{a}$, and gave $\mathbf{1} \mathbf{g}$ in $54 \%$ yield as a white solid; m.p. 250.0-251.2 ${ }^{\circ} \mathrm{C} ;{ }^{1} \mathrm{H}$ NMR $\left(500 \mathrm{MHz}, \mathrm{DMF}-\mathrm{d}_{7}\right) \delta 12.29$ (brs, $\left.1 \mathrm{H}\right), 7.65-7.57(\mathrm{~m}, 5 \mathrm{H}), 7.51-7.47(\mathrm{~m}, 3 \mathrm{H}), 7.32$ (dd, $\left.1 \mathrm{H}, J_{1}=J_{2}=7.4 \mathrm{~Hz}\right), 7.20-7.15(\mathrm{~m}, 3 \mathrm{H}), 7.07$ (dd, $\left.1 \mathrm{H}, \mathrm{J}=7.4 \mathrm{~Hz}\right) ;{ }^{13} \mathrm{C}$ NMR $(126 \mathrm{MHz}$, DMF- $\left.d_{7}\right) \delta 192.9,142.4,140.8,137.3,137.3,132.6,132.4,131.1,130.1,129.1,128.7,124.2$, 122.4, 121.7, 119.2, 114.6, 112.8, 111.9; IR (ATR) 3114, 2230, 1593, 1566, 1418, $1210 \mathrm{~cm}^{-1}$; MS (FAB+) $m / z=323\left([M+H]^{+}\right)$; HRMS (FAB+) Calcd for $\mathrm{C}_{22} \mathrm{H}_{15} \mathrm{~N}_{2} \mathrm{O}[\mathrm{M}+\mathrm{H}]^{+}: 323.1184$; found:323.1192 


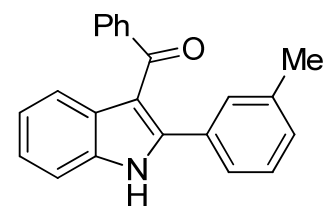

Phenyl(2-(m-tolyl)-1H-indol-3-yl)methanone (1h): The reaction was performed in DMF at $100{ }^{\circ} \mathrm{C}$ according to the procedure for $\mathbf{1 a}$, and gave $\mathbf{1 h}$ in $85 \%$ yield as a white solid; m.p. 153.0-154.2 ${ }^{\circ} \mathrm{C} ;{ }^{1} \mathrm{H}$ NMR (500 MHz, DMF- $\left.d_{7}\right) \delta 11.98$ (brs, $\left.1 \mathrm{H}\right), 7.70(\mathrm{~d}, 1 \mathrm{H}, \mathrm{J}=8.0 \mathrm{~Hz}$ ), 7.44$7.41(\mathrm{~m}, 3 \mathrm{H}), 7.21\left(\mathrm{dd}, 1 \mathrm{H}, \mathrm{J}_{1}=\mathrm{J}_{2}=7.4 \mathrm{~Hz}\right), 7.15-7.06(\mathrm{~m}, 5 \mathrm{H}), 7.04-6.98(\mathrm{~m}, 2 \mathrm{H}), 6.90(\mathrm{~d}, 1 \mathrm{H}$, $\mathrm{J}=7.4 \mathrm{~Hz}), 2.01(\mathrm{~s}, 3 \mathrm{H}) ;{ }^{13} \mathrm{C}$ NMR $\left(126 \mathrm{MHz}, \mathrm{DMF}-d_{7}\right) \delta 193.2,145.1,141.2,138.2,137.0$, $132.5,131.9,131.3,129.8,129.7,129.4,128.7,128.4,127.2,123.6,122.0,121.5,113.3$, 112.4, 21.0; IR (ATR) 3210, 1593, 1570, 1447, 1429, 1209, $887 \mathrm{~cm}^{-1}$; MS (FAB+) m/z = 312 $\left([\mathrm{M}+\mathrm{H}]^{+}\right)$; HRMS (FAB+) Calcd for $\mathrm{C}_{22} \mathrm{H}_{16} \mathrm{NO}[\mathrm{M}+\mathrm{H}]^{+}: 312.1388$; found:312.1387.

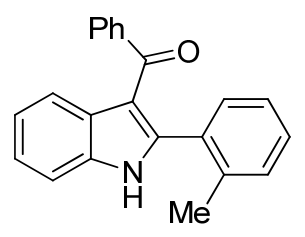

Phenyl(2-(o-tolyl)-1H-indol-3-yl)methanone (1i): The reaction was performed in DMF at 100 ${ }^{\circ} \mathrm{C}$ according to the procedure for $\mathbf{1 a}$, and gave $\mathbf{1 i}$ in $85 \%$ yield as a white solid; m.p. 211.2$212.2{ }^{\circ} \mathrm{C} ;{ }^{1} \mathrm{H}$ NMR (500 MHz, DMF- $\left.d_{7}\right) \delta 12.00$ (brs, $\left.1 \mathrm{H}\right), 7.94(\mathrm{~d}, 1 \mathrm{H}, \mathrm{J}=8.0 \mathrm{~Hz}), 7.58-7.53(\mathrm{~m}$, $3 \mathrm{H}), 7.34-7.12(\mathrm{~m}, 8 \mathrm{H}), 7.06\left(\mathrm{dd}, 1 \mathrm{H}, \mathrm{J}_{1}=\mathrm{J}_{2}=7.4 \mathrm{~Hz}\right), 2.25(\mathrm{~s}, 3 \mathrm{H}) ;{ }^{13} \mathrm{C}$ NMR (126 MHz, DMF$\left.d_{7}\right) \delta 192.8,145.1,141.3,137.4,136.8,133.2,131.9,131.5,130.5,129.4,129.3,128.7,128.2$, 125.9, 123.5, 122.1, 121.8, 114.7, 112.4, 20.2; IR (ATR) 3056, 1591, 1563, 1445, 1423, 1209 , $883 \mathrm{~cm}^{-1}$; MS (FAB+) $\mathrm{m} / z=312\left([\mathrm{M}+\mathrm{H}]^{+}\right)$; HRMS (FAB+) Calcd for $\mathrm{C}_{22} \mathrm{H}_{18} \mathrm{NO}$ $[\mathrm{M}+\mathrm{H}]^{+}: 312.1388$; found:312.1387.<smiles>O=C(c1ccccc1)c1c(-c2ccccc2)[nH]c2ccc(Cl)cc12</smiles>

(5-Chloro-2-phenyl-1H-indol-3-yl)(phenyl)methanone (1j): The reaction was performed in DMF at $100{ }^{\circ} \mathrm{C}$ according to the procedure for $1 \mathrm{a}$ with $10 \mathrm{~mol} \%$ of $\mathrm{Pd}(\mathrm{OAc})_{2}$ and $20 \mathrm{~mol} \%$ of $\mathrm{Ad}_{2} \mathrm{P}^{n} \mathrm{Bu}$, and gave $1 \mathrm{j}$ in $41 \%$ yield as a white solid; m.p. $261.8-263.6{ }^{\circ} \mathrm{C} ;{ }^{1} \mathrm{H}$ NMR $(500 \mathrm{MHz}$, 
DMF- $\left.d_{7}\right) \delta 12.28($ brs, $1 \mathrm{H}), 7.74(\mathrm{~s}, 1 \mathrm{H}), 7.48-7.46(\mathrm{~m}, 3 \mathrm{H}), 7.33(\mathrm{~d}, 2 \mathrm{H}, \mathrm{J}=6.9 \mathrm{~Hz}), 7.24$ (dd, $\left.1 \mathrm{H}, J_{1}=J_{2}=7.4 \mathrm{~Hz}\right), 7.15-7.11(\mathrm{~m}, 6 \mathrm{H}) ;{ }^{13} \mathrm{C}$ NMR $\left(126 \mathrm{MHz}, \mathrm{DMF}-d_{7}\right) \delta 192.8,146.4,140.6$, $135.5,132.3,132.1,130.5,130.4,129.9,129.4,128.8,128.5,127.2,123.7,120.7,114.1$, 112.9; IR (ATR) 3160, 1595, 1563, 1409, $1215 \mathrm{~cm}^{-1}$; MS (FAB+) $\mathrm{m} / \mathrm{z}=332\left([\mathrm{M}+\mathrm{H}]^{+}\right)$; HRMS $(\mathrm{FAB}+)$ Calcd for $\mathrm{C}_{21} \mathrm{H}_{15} \mathrm{CINO}[\mathrm{M}+\mathrm{H}]^{+}: 332.0842$; found: 332.0837 .

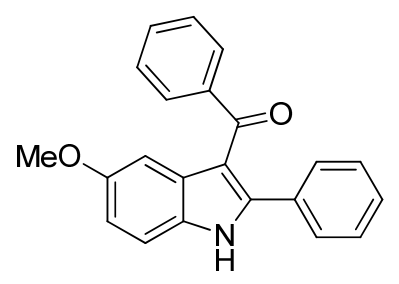

(5-Methoxy-2-phenyl-1H-indol-3-yl)(phenyl)methanone (1k): The reaction was performed in DMF at $100{ }^{\circ} \mathrm{C}$ according to the procedure for $\mathbf{1 a}$, and gave $\mathbf{1 k}$ in $69 \%$ yield as a white solid; m.p. 182.0-183.2 ${ }^{\circ} \mathrm{C} ;{ }^{1} \mathrm{H}$ NMR (500 MHz, DMF- $\left.d_{7}\right) \delta 12.06$ (brs, $\left.1 \mathrm{H}\right), 7.60(\mathrm{~d}, 2 \mathrm{H}, \mathrm{J}=8.0 \mathrm{~Hz})$, 7.50-7.42 (m, 4H), $7.37\left(\mathrm{dd}, 1 \mathrm{H}, \mathrm{J}_{1}=\mathrm{J}_{2}=7.4 \mathrm{~Hz}\right), 7.25-7.22(\mathrm{~m}, 5 \mathrm{H}), 6.94\left(\mathrm{dd}, 1 \mathrm{H}, \mathrm{J}_{1}=8.9 \mathrm{~Hz}\right.$, $\left.J_{2}=2.6 \mathrm{~Hz}\right), 3.80(\mathrm{~s}, 3 \mathrm{H}) ;{ }^{13} \mathrm{C}$ NMR $\left(126 \mathrm{MHz}, \mathrm{DMF}-d_{7}\right) \delta 193.0,156.3,145.4,141.2,132.9$, 132.0, 131.8, 130.3, 130.2, 129.9, ,129.0, 128.7, 128.4, 113.6, 113.2, 103.3, 55.7 (One carbon peak was overlapping) ; IR (ATR) 3173, 1597, 1414, $1271 \mathrm{~cm}^{-1}$; MS (FAB+) $\mathrm{m} / \mathrm{z}=328$ $\left([\mathrm{M}+\mathrm{H}]^{+}\right)$; HRMS $(\mathrm{FAB}+)$ Calcd for $\mathrm{C}_{22} \mathrm{H}_{18} \mathrm{NO}_{2}[\mathrm{M}+\mathrm{H}]^{+}: 328.1338$; found:328.1347.

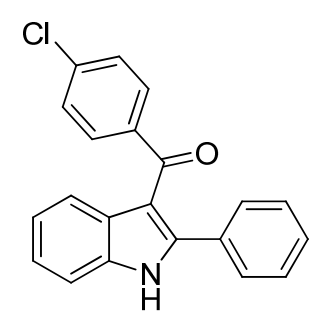

(4-Chlorophenyl)(2-phenyl-1H-indol-3-yl)methanone (1I): The reaction was performed in DMF at $100{ }^{\circ} \mathrm{C}$ according to the procedure for $\mathbf{1 a}$, and gave $\mathbf{1}$ in $47 \%$ yield as a white solid; m.p. 226.4-228.0 ${ }^{\circ} \mathrm{C} ;{ }^{1} \mathrm{H}$ NMR (500 MHz, DMF- $\left.d_{7}\right) \delta 12.23$ (brs, $\left.1 \mathrm{H}\right), 7.91$ (d, $1 \mathrm{H}, \mathrm{J}=8.0 \mathrm{~Hz}$ ), 7.61-7.59 (m, 3H), $7.47(\mathrm{~d}, 2 \mathrm{H}, \mathrm{J}=6.0 \mathrm{~Hz}), 7.29-7.20(\mathrm{~m}, 7 \mathrm{H}) ;{ }^{13} \mathrm{C}$ NMR $\left(126 \mathrm{MHz}, \mathrm{DMF}-d_{7}\right) \delta$ 191.7, 145.2, 139.8, 137.1, 137.1, 132.6, 131.8, 130.5, 129.3, 129.2, ,128.8, 128.6, 123.7, 122.2, 121.5, 113.1, 112.5; IR (ATR) 3133, 1592, 1445, 1412, $1274 \mathrm{~cm}^{-1}$; MS (FAB+) $\mathrm{m} / \mathrm{z}=$ $332\left([\mathrm{M}+\mathrm{H}]^{+}\right)$; HRMS (FAB+) Calcd for $\mathrm{C}_{21} \mathrm{H}_{15} \mathrm{CINO}[\mathrm{M}+\mathrm{H}]^{+}: 332.0842$; found:332.0837. 


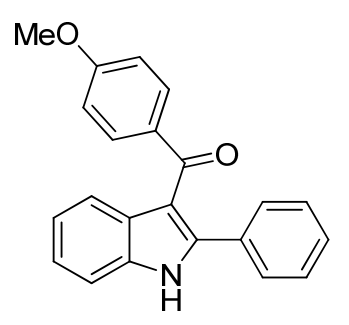

(4-Methoxyphenyl)(2-phenyl-1H-indol-3-yl)methanone (1m): The reaction was performed in DMF at $100^{\circ} \mathrm{C}$ according to the procedure for $1 \mathrm{a}$, and gave $1 \mathrm{~m}$ in $60 \%$ yield as a white solid; m.p. 152.7-154.0 ${ }^{\circ} \mathrm{C} ;{ }^{1} \mathrm{H}$ NMR (500 MHz, DMF- $\left.d_{7}\right) \delta 12.10$ (brs, $\left.1 \mathrm{H}\right), 7.71-7.67(\mathrm{~m}, 3 \mathrm{H}), 7.58-$ $7.54(\mathrm{~m}, 3 \mathrm{H}), 7.33-7.24(\mathrm{~m}, 4 \mathrm{H}), 7.15\left(\mathrm{dd}, 1 \mathrm{H}, J_{1}=J_{2}=7.4 \mathrm{~Hz}\right), 6.85(\mathrm{~d}, 2 \mathrm{H}, \mathrm{J}=8.9 \mathrm{~Hz}), 3.80$ $(\mathrm{s}, 3 \mathrm{H}) ;{ }^{13} \mathrm{C}$ NMR $\left(126 \mathrm{MHz}, \mathrm{DMF}-d_{7}\right) \delta 191.9,163.3,143.3,137.0,133.3,132.9,132.3,130.0$, 129.4, 129.0, 128.9, 123.4, 121.6, 121.2, 113.9, 113.6, 112.4, 55.8; IR (ATR) 3184, 1592, 1423, $1251 \mathrm{~cm}^{-1}$; MS (FAB+) $\mathrm{m} / \mathrm{z}=328\left([\mathrm{M}+\mathrm{H}]^{+}\right)$; HRMS (FAB+) Calcd for $\mathrm{C}_{22} \mathrm{H}_{17} \mathrm{NO}_{2}$ $[\mathrm{M}]^{+}: 327.1259$; found:327.1252.

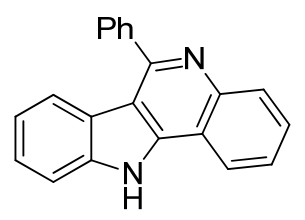

6-Phenyl-11H-indolo[3,2-c]quinoline (5): The reaction was performed in toluene at $100{ }^{\circ} \mathrm{C}$ according to the procedure for $1 \mathbf{a}$ with $10 \mathrm{~mol} \%$ of $\mathrm{Pd}(\mathrm{OAc})_{2}$ and $20 \mathrm{~mol} \%$ of $\mathrm{Ad}_{2} \mathrm{P}^{n} \mathrm{Bu}$ to give 5 in $56 \%$ yield as a orange solid; m.p. $237.0-238.4{ }^{\circ} \mathrm{C} ;{ }^{1} \mathrm{H}$ NMR $\left(500 \mathrm{MHz}\right.$, DMSO- $\left.d_{6}\right) \delta 8.57(\mathrm{~d}$, $1 \mathrm{H}, J=8.0 \mathrm{~Hz}$ ), $8.13(\mathrm{~d}, 1 \mathrm{H}, \mathrm{J}=8.3 \mathrm{~Hz}), 7.82(\mathrm{~d}, 2 \mathrm{H}, J=7.4 \mathrm{~Hz}), 7.80-7.57(\mathrm{~m}, 6 \mathrm{H}), 7.50(\mathrm{~d}$, $1 \mathrm{H}, J=8.0 \mathrm{~Hz}), 7.43(\mathrm{t}, 1 \mathrm{H}, \mathrm{J}=7.7 \mathrm{~Hz}), 7.12(\mathrm{t}, 1 \mathrm{H}, \mathrm{J}=7.6 \mathrm{~Hz}) ;{ }^{13} \mathrm{C}$ NMR $(126 \mathrm{MHz}$, DMSO$\left.d_{6}\right) \delta 155.5,144.9,141.0,140.7,139.0,129.4,128.8,128.5,128.4,125.6,125.3,121.9,121.7$, 121.0, 120.2, 116.3, 111.9 (Two carbon peak was overlapping); IR (ATR) 3057, 1558, 1512, $1361,1242 \mathrm{~cm}^{-1}$; MS (FAB+) $\mathrm{mlz}=295\left([\mathrm{M}+\mathrm{H}]^{+}\right)$; HRMS $(\mathrm{FAB}+)$ Calcd for $\mathrm{C}_{21} \mathrm{H}_{15} \mathrm{~N}_{2}[\mathrm{M}+\mathrm{H}]^{+}$: 295.1235; found: 295.1233. The spectroscopic data were in agreement with those reported in the literature. ${ }^{5}$ 


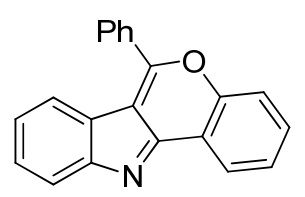

6-Phenylchromeno[4,3-b]indole (7): The reaction was performed in toluene at $100{ }^{\circ} \mathrm{C}$ according to the procedure for $1 \mathrm{a}$ with $10 \mathrm{~mol} \%$ of $\mathrm{Pd}(\mathrm{OAc})_{2}$ and $20 \mathrm{~mol} \%$ of $\mathrm{Ad}_{2} \mathrm{P}^{n} \mathrm{Bu}$ to give 7 in 73\% yield as a yellow solid; m.p. $143.3-144.7^{\circ} \mathrm{C} ;{ }^{1} \mathrm{H} \mathrm{NMR}(500 \mathrm{MHz}, \mathrm{CDCl}) \delta: 8.70(1 \mathrm{H}, \mathrm{d}$, $J=7.9 \mathrm{~Hz}), 8.06-8.03(2 \mathrm{H}, \mathrm{m}), 7.94(1 \mathrm{H}, \mathrm{d}, J=8.0 \mathrm{~Hz}), 7.82(1 \mathrm{H}, \mathrm{d}, J=7.7 \mathrm{~Hz}), 7.75-7.65(5 \mathrm{H}$, $\mathrm{m}), 7.58(1 \mathrm{H}, \mathrm{t}, \mathrm{J}=7.4 \mathrm{~Hz}), 7.51(1 \mathrm{H}, \mathrm{t}, \mathrm{J}=7.7 \mathrm{~Hz}), 7.19(1 \mathrm{H}, \mathrm{t}, \mathrm{J}=7.4 \mathrm{~Hz}) ;{ }^{13} \mathrm{C}$ NMR $(500$ $\mathrm{MHz}, \mathrm{CDCl} 3) \delta$ : 158.2, 156.5, 155.3, 152.5, 132.5, 131.7, 131.0, 130.0, 129.2, 129.0, 128.4, 126.0, 125.2, 124.4, 122.7, 121.3, 120.0, 118.7, 118.1; IR (ATR) 3056, 1638, 1378, $1056 \mathrm{~cm}^{-1}$; MS $(F A B+) m / z=296\left([M+H]^{+}\right)$; HRMS $(F A B+)$ Calcd for $\mathrm{C}_{21} \mathrm{H}_{14} \mathrm{NO}[\mathrm{M}+\mathrm{H}]^{+}: 296.1075$; found: 296.1075 .

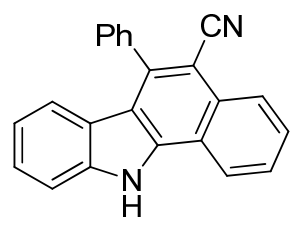

6-Phenyl-11H-benzo[a]carbazole-5-carbonitrile (9): The reaction was performed in DMF at $100{ }^{\circ} \mathrm{C}$ according to the procedure for $1 \mathrm{a}$ with $10 \mathrm{~mol} \%$ of $\mathrm{Pd}(\mathrm{OAc})_{2}$ and $20 \mathrm{~mol} \%$ of $\mathrm{Ad}_{2} \mathrm{P}^{n} \mathrm{Bu}$ to give 9 in $68 \%$ yield as a white solid; m.p. $282.0-282.8^{\circ} \mathrm{C} ;{ }^{1} \mathrm{H}$ NMR $\left(500 \mathrm{MHz}\right.$, DMF- $\left.d_{7}\right) \delta$ $13.07(1 \mathrm{H}, \mathrm{brs}), 8.68(1 \mathrm{H}, \mathrm{d}, \mathrm{J}=8.0 \mathrm{~Hz}), 8.21(1 \mathrm{H}, \mathrm{d}, \mathrm{J}=8.0 \mathrm{~Hz}), 7.77-7.70(2 \mathrm{H}, \mathrm{m}), 7.62-7.55$ $(6 \mathrm{H}, \mathrm{m}), 7.29(1 \mathrm{H}, \mathrm{t}, \mathrm{J}=7.7 \mathrm{~Hz}), 6.91(1 \mathrm{H}, \mathrm{t}, \mathrm{J}=7.4 \mathrm{~Hz}), 6.80(1 \mathrm{H}, \mathrm{d}, \mathrm{J}=8.0 \mathrm{~Hz}) ;{ }^{13} \mathrm{C}$ NMR $\left(500 \mathrm{MHz}, \mathrm{DMF}-d_{7}\right) \delta: 144.5,140.6,138.9,138.7,131.5,129.9,129.7,129.6,128.8,127.6$, 126.2, 123.6, 123.4, 121.7, 121.1, 120.9, 118.8, 116.8, 112.7, 100.6 (One carbon peak was overlapping); IR (ATR) 3273, 2213, $1371 \mathrm{~cm}^{-1}$; MS (FAB+) $\mathrm{m} / \mathrm{z}=319\left([\mathrm{M}+\mathrm{H}]^{+}\right)$; HRMS (FAB+) Calcd for $\mathrm{C}_{23} \mathrm{H}_{14} \mathrm{~N}_{2}[\mathrm{M}]^{+}: 318.1157$; found: 318.1166 . The structure of 9 was determined $X$-ray crystallography. 


\section{X-ray structure of 9}

\section{Data Collection}

A colorless platelet crystal of $\mathrm{C}_{47} \mathrm{H}_{29} \mathrm{Cl}_{3} \mathrm{~N}_{4}$ having approximate dimensions of $0.800 \mathrm{x}$ $0.500 \times 0.150 \mathrm{~mm}$ was mounted on a glass fiber. All measurements were made on a Rigaku R-AXIS RAPID diffractometer using graphite monochromated Mo-K $\alpha$ radiation.

The crystal-to-detector distance was $127.40 \mathrm{~mm}$.

Cell constants and an orientation matrix for data collection corresponded to a primitive triclinic cell with dimensions:

$$
\begin{array}{ll}
a=7.366(2) \AA & \alpha=64.891(4)^{\mathrm{O}} \\
\mathrm{b}=16.750(3) \AA & \beta=81.409(4)^{\mathrm{O}} \\
\mathrm{c}=17.108(3) \AA & \gamma=78.963(4)^{\mathrm{O}} \\
\mathrm{V}=1870.4(5) \AA^{3} &
\end{array}
$$

For $Z=2$ and F.W. = 756.13, the calculated density is $1.342 \mathrm{~g} / \mathrm{cm}^{3}$. Based on a statistical analysis of intensity distribution, and the successful solution and refinement of the structure, the space group was determined to be:

$$
\text { P-1 (\#2) }
$$

The data were collected at a temperature of $23 \pm 1^{\circ} \mathrm{C}$ to a maximum $2 \theta$ value of $55.0^{\circ}$. A total of 44 oscillation images were collected. A sweep of data was done using $\omega$ scans from 130.0 to $190.0^{\circ}$ in $5.0^{\circ}$ step, at $\chi=45.0^{\circ}$ and $\phi=0.0^{\circ}$. The exposure rate was 120.0 [sec./O]. A second sweep was performed using $\omega$ scans from 0.0 to $160.0^{\circ}$ in $5.0^{\circ}$ step, at $\chi=45.0^{\circ}$ and $\phi$ $=180.0^{\circ}$. The exposure rate was 120.0 [sec./O]. The crystal-to-detector distance was 127.40 $\mathrm{mm}$. Readout was performed in the $0.100 \mathrm{~mm}$ pixel mode. 


\section{Data Reduction}

Of the 16147 reflections that were collected, 7745 were unique $\left(R_{\text {int }}=0.1109\right)$; equivalent reflections were merged.

The linear absorption coefficient, $\mu$, for Mo-K $\alpha$ radiation is $2.852 \mathrm{~cm}^{-1}$. An empirical absorption correction was applied which resulted in transmission factors ranging from 0.257 to 0.958. The data were corrected for Lorentz and polarization effects. A correction for secondary extinction $^{1}$ was applied (coefficient $\left.=0.008910\right)$.

\section{$\underline{\text { Structure Solution and Refinement }}$}

The structure was solved by direct methods 2 and expanded using Fourier techniques. The non-hydrogen atoms were refined anisotropically. Hydrogen atoms were refined using the riding model. The final cycle of full-matrix least-squares refinement ${ }^{3}$ on $\mathrm{F}^{2}$ was based on 7745 observed reflections and 488 variable parameters and converged (largest parameter shift was 0.00 times its esd) with unweighted and weighted agreement factors of:

$$
\begin{gathered}
\mathrm{R} 1=\Sigma\|\mathrm{Fo}|-| \mathrm{Fc}\| / \Sigma|\mathrm{Fo}|=0.0838 \\
\mathrm{wR} 2=\left[\Sigma\left(\mathrm{w}\left(\mathrm{Fo}^{2}-\mathrm{Fc}^{2}\right)^{2}\right) / \Sigma \mathrm{w}\left(\mathrm{Fo}^{2}\right)^{2}\right]^{1 / 2}=0.2326
\end{gathered}
$$

The standard deviation of an observation of unit weight ${ }^{4}$ was 1.03. Unit weights were used. The maximum and minimum peaks on the final difference Fourier map corresponded to 0.47 and $-0.43 e^{-} / \AA^{3}$, respectively.

Neutral atom scattering factors were taken from Cromer and Waber 5 . Anomalous dispersion effects were included in Fcalc 6 ; the values for $\Delta f^{\prime}$ and $\Delta f$ " were those of Creagh and McAuley 7 . The values for the mass attenuation coefficients are those of Creagh and Hubbell 8 . 


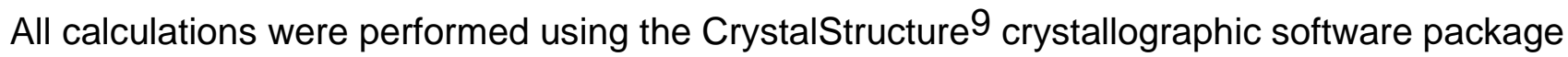
except for refinement, which was performed using SHELXL-9710.

\section{References}

(1) Larson, A.C. (1970), Crystallographic Computing, 291-294. F.R. Ahmed, ed. Munksgaard, Copenhagen (equation 22, with $\mathrm{V}$ replaced by the cell volume).

(2) SIR2008: M.C. Burla, R. Caliandro, M. Camalli, B. Carrozzini, G.L. Cascarano, L. De Caro, C. Giacovazzo, G. Polidori, D. Siliqi, R. Spagna (2007)

(3) Least Squares function minimized: (SHELXL97)

$$
\Sigma w\left(F_{0}^{2}-F_{c}^{2}\right)^{2} \quad \text { where } w=\text { Least Squares weights. }
$$

(4) Standard deviation of an observation of unit weight:

$$
\begin{aligned}
& {\left[\Sigma w\left(\mathrm{~F}_{0}{ }^{2}-\mathrm{F}_{\mathrm{C}}{ }^{2}\right)^{2} /\left(\mathrm{N}_{\mathrm{O}}-\mathrm{N}_{\mathrm{V}}\right)\right]^{1 / 2}} \\
& \text { where: } \quad \begin{aligned}
\mathrm{N}_{\mathrm{O}} & =\text { number of observations } \\
\mathrm{N}_{\mathrm{V}} & =\text { number of variables }
\end{aligned}
\end{aligned}
$$

(5) Cromer, D. T. \& Waber, J. T.; "International Tables for X-ray Crystallography", Vol. IV, The Kynoch Press, Birmingham, England, Table 2.2 A (1974).

(6) Ibers, J. A. \& Hamilton, W. C.; Acta Crystallogr., 17, 781 (1964).

(7) Creagh, D. C. \& McAuley, W.J .; "International Tables for Crystallography", Vol C, (A.J.C. Wilson, ed.), Kluwer Academic Publishers, Boston, Table 4.2.6.8, pages 219-222 (1992).

(8) Creagh, D. C. \& Hubbell, J.H..; "International Tables for Crystallography", Vol C, (A.J.C. 
Wilson, ed.), Kluwer Academic Publishers, Boston, Table 4.2.4.3, pages 200-206 (1992).

(9) CrystalStructure 4.0: Crystal Structure Analysis Package, Rigaku Corporation (2000-2010). Tokyo 196-8666, Japan.

(10) SHELX97: Sheldrick, G.M. (2008). Acta Cryst. A64, 112-122. 


\section{A. Crystal Data}

Empirical Formula

Formula Weight

Crystal Color, Habit

Crystal Dimensions

Crystal System

Lattice Type

Lattice Parameters

Space Group

$Z$ value

$D_{\text {calc }}$

$F_{000}$

$\mu(\operatorname{MoK} \alpha)$
$\mathrm{C}_{47} \mathrm{H}_{29} \mathrm{Cl}_{3} \mathrm{~N}_{4}$

756.13

colorless, platelet

$0.800 \times 0.500 \times 0.150 \mathrm{~mm}$

triclinic

Primitive

$$
\begin{aligned}
& a=7.366(2) \AA \\
& b=16.750(3) \AA \\
& c=17.108(3) \AA \\
& \alpha=64.891(4) 0 \\
& \beta=81.409(4) 0 \\
& \gamma=78.963(4) 0 \\
& V=1870.4(5) \AA^{3}
\end{aligned}
$$

P-1 (\#2)

2

$1.342 \mathrm{~g} / \mathrm{cm}^{3}$

780.00

$2.852 \mathrm{~cm}^{-1}$ 

B. Intensity Measurements

Diffractometer

Radiation

Voltage, Current

Temperature

Detector Aperture

Data Images

$\omega$ oscillation Range $(\chi=45.0, \phi=0.0)$

Exposure Rate

$\omega$ oscillation Range $(\chi=45.0, \phi=180.0)$

Exposure Rate

Detector Position

Pixel Size

$2 \theta_{\max }$

No. of Reflections Measured

Corrections
R-AXIS RAPID

$\operatorname{MoK} \alpha(\lambda=0.71075 \AA)$

graphite monochromated

$50 \mathrm{kV}, 40 \mathrm{~mA}$

$23.0^{\circ} \mathrm{C}$

$280 \times 256 \mathrm{~mm}$

44 exposures

$130.0-190.0^{0}$

$120.0 \mathrm{sec} . / 0$

$0.0-160.0^{0}$

$120.0 \mathrm{sec} . / 0$

$127.40 \mathrm{~mm}$

$0.100 \mathrm{~mm}$

$55.0^{0}$

Total: 16147

Unique: $7745\left(R_{\text {int }}=0.1109\right)$

Lorentz-polarization 
Absorption

(trans. factors: 0.257 - 0.958)

Secondary Extinction

(coefficient: 8.91000e-003) 
C. Structure Solution and Refinement

Structure Solution

Refinement

Function Minimized

Least Squares Weights

$2 \theta_{\max }$ cutoff

Anomalous Dispersion

No. Observations (All reflections)

No. Variables

Reflection/Parameter Ratio

Residuals: R1 $(\mathrm{I}>2.00 \sigma(\mathrm{I}))$

Residuals: R (All reflections)

Residuals: wR2 (All reflections)

Goodness of Fit Indicator

Max Shift/Error in Final Cycle

Maximum peak in Final Diff. Map
Direct Methods

Full-matrix least-squares on $\mathrm{F}^{2}$

$\Sigma w\left(\mathrm{Fo}^{2}-\mathrm{Fc}^{2}\right)^{2}$

$w=1 /\left[\sigma^{2}\left(F_{0}^{2}\right)+(0.0907 \cdot P)^{2}\right.$

$+1.1274 \cdot \mathrm{P}$ ]

where $\mathrm{P}=\left(\operatorname{Max}\left(\mathrm{Fo}^{2}, 0\right)+2 \mathrm{Fc}^{2}\right) / 3$

$55.0^{\circ}$

All non-hydrogen atoms

7745

488

15.87

0.0838

0.1730

0.2326

1.028

0.000

$0.47 e^{-/ \AA^{3}}$

S20 
Table 1. Atomic coordinates and $\mathrm{B}_{\mathrm{iso}} / \mathrm{B}_{\mathrm{eq}}$

$\begin{array}{lllll}\text { atom } & \mathrm{x} & \mathrm{y} & \mathrm{z} & \mathrm{B} \text { eq } \\ \mathrm{Cl} 1 & 0.5697(2) & 0.25424(9) & 0.29686(9) & 3.34(3) \\ \mathrm{Cl} 2 & 0.9373(2) & 0.1629(2) & 0.2647(1) & 4.78(4) \\ \mathrm{Cl} 3 & 0.8500(3) & 0.3576(2) & 0.1789(1) & 5.02(4) \\ \mathrm{N} 1 & 0.2682(5) & 0.3625(3) & 0.5501(3) & 1.98(7) \\ \mathrm{N} 2 & 0.1425(6) & 0.7670(3) & 0.2192(3) & 2.63(8) \\ \mathrm{N} 3 & 0.6867(5) & 0.1490(3) & -0.0396(3) & 2.14(7) \\ \text { N4 } & 0.6191(6) & -0.2613(3) & 0.2713(3) & 2.71(8) \\ \text { C1 } & 0.2445(6) & 0.3062(3) & 0.5127(3) & 1.96(8) \\ \text { C2 } & 0.2567(6) & 0.2129(3) & 0.5500(3) & 2.22(9) \\ \text { C3 } & 0.2193(7) & 0.1721(3) & 0.4996(4) & 2.6(1) \\ \text { C4 } & 0.1700(7) & 0.2240(3) & 0.4127(3) & 2.35(9) \\ \text { C5 } & 0.1598(6) & 0.3156(3) & 0.3769(3) & 2.08(9) \\ \text { C6 } & 0.2007(6) & 0.3592(3) & 0.4254(3) & 1.77(8) \\ \text { C7 } & 0.2040(6) & 0.4509(3) & 0.4120(3) & 1.70(8) \\ \text { C8 } & 0.1778(6) & 0.5345(3) & 0.3380(3) & 1.72(8) \\ \text { C9 } & 0.1870(6) & 0.6110(3) & 0.3498(3) & 1.87(8) \\ \text { C10 } & 0.2247(6) & 0.6109(3) & 0.4303(3) & 1.66(8) \\ \text { C11 } & 0.2383(6) & 0.6900(3) & 0.4399(3) & 2.07(9) \\ \text { C12 } & 0.2773(7) & 0.6868(4) & 0.5177(3) & 2.44(9) \\ \text { C13 } & 0.3085(7) & 0.6040(3) & 0.5889(3) & 2.33(9) \\ \text { C14 } & 0.2977(6) & 0.5260(4) & 0.5815(3) & 2.11(9) \\ \text { C15 } & 0.2560(6) & 0.5269(3) & 0.5033(3) & 1.79(8) \\ \text { C16 } & 0.2442(6) & 0.4499(3) & 0.4899(3) & 1.54(8) \\ \text { C17 } & 0.1625(6) & 0.6966(3) & 0.2764(3) & 2.02(8) \\ \text { C18 } & 0.1545(7) & 0.5358(3) & 0.2525(3) & 2.05(9) \\ \text { C19 } & -0.0091(7) & 0.5787(3) & 0.2088(3) & 2.5(1) \\ \text { C20 } & -0.0226(8) & 0.5787(4) & 0.1280(4) & 2.9(1) \\ \text { C21 } & 0.1245(8) & 0.5358(4) & 0.0906(4) & 3.1(1)\end{array}$




$\begin{array}{lllll}\text { C22 } & 0.2869(8) & 0.4927(3) & 0.1344(3) & 2.7(1) \\ \text { C23 } & 0.3017(7) & 0.4926(3) & 0.2142(3) & 2.24(9) \\ \text { C24 } & 0.5895(7) & 0.2049(3) & -0.0008(3) & 2.22(9) \\ \text { C25 } & 0.5538(7) & 0.2980(3) & -0.0360(3) & 2.55(9) \\ \text { C26 } & 0.4417(7) & 0.3388(4) & 0.0141(4) & 2.7(1) \\ \text { C27 } & 0.3674(7) & 0.2875(3) & 0.0981(3) & 2.52(9) \\ \text { C28 } & 0.4073(7) & 0.1949(3) & 0.1334(3) & 2.12(9) \\ \text { C29 } & 0.5217(6) & 0.1518(3) & 0.0843(3) & 1.88(8) \\ \text { C30 } & 0.5862(6) & 0.0594(3) & 0.0962(3) & 1.90(8)\end{array}$


Table 1. Atomic coordinates and $\mathrm{B}_{\text {iso }} / \mathrm{B}_{\text {eq }}$ (continued)

\begin{tabular}{|c|c|c|c|c|}
\hline atom & $x$ & $y$ & z & $\mathrm{B}_{\mathrm{eq}}$ \\
\hline C31 & $0.5675(6)$ & $-0.0248(3)$ & $0.1657(3)$ & $1.85(8)$ \\
\hline C32 & $0.6402(6)$ & $-0.1023(3)$ & $0.1519(3)$ & $1.85(8)$ \\
\hline C33 & $0.7408(6)$ & $-0.1000(3)$ & $0.0711(3)$ & $2.01(9)$ \\
\hline C34 & $0.8196(6)$ & $-0.1785(3)$ & $0.0576(3)$ & $2.04(9)$ \\
\hline C35 & $0.9165(7)$ & $-0.1736(4)$ & $-0.0195(3)$ & $2.55(9)$ \\
\hline C36 & $0.9395(7)$ & $-0.0893(4)$ & $-0.0865(3)$ & $2.6(1)$ \\
\hline C37 & $0.8646(7)$ & $-0.0128(4)$ & $-0.0760(3)$ & $2.31(9)$ \\
\hline C38 & $0.7638(6)$ & $-0.0147(3)$ & $0.0020(3)$ & $1.93(8)$ \\
\hline C39 & $0.6844(6)$ & $0.0617(3)$ & $0.0179(3)$ & $1.86(8)$ \\
\hline $\mathrm{C} 40$ & $0.6258(7)$ & $-0.1893(4)$ & $0.2194(3)$ & $2.23(9)$ \\
\hline C41 & $0.4721(7)$ & $-0.0285(3)$ & $0.2508(3)$ & $2.11(9)$ \\
\hline C42 & $0.5435(7)$ & $0.0126(3)$ & $0.2940(3)$ & $2.42(9)$ \\
\hline $\mathrm{C} 43$ & $0.4545(8)$ & $0.0132(4)$ & $0.3720(4)$ & $3.1(1)$ \\
\hline C44 & $0.2952(8)$ & $-0.0276(3)$ & $0.4080(4)$ & $2.9(1)$ \\
\hline C45 & $0.2218(7)$ & $-0.0692(4)$ & $0.3663(4)$ & $2.9(1)$ \\
\hline C46 & $0.3123(7)$ & $-0.0705(3)$ & $0.2876(3)$ & $2.45(9)$ \\
\hline C47 & $0.7587(8)$ & $0.2556(4)$ & $0.2193(4)$ & $3.7(2)$ \\
\hline
\end{tabular}


Table 2. Atomic coordinates and $\mathrm{B}_{\text {iso }}$ involving hydrogen atoms

$\begin{array}{lcccc}\text { atom } & x & y & z & B \\ \text { H1 } & 0.2938 & 0.3455 & 0.6027 & 2.38 \\ \text { H2 } & 0.2889 & 0.1795 & 0.6066 & 2.67 \\ \text { H3 } & 0.7396 & 0.1666 & -0.0912 & 2.57 \\ \text { H3A } & 0.2263 & 0.1105 & 0.5226 & 3.11 \\ \text { H4 } & 0.1446 & 0.1958 & 0.3798 & 2.82 \\ \text { H5 } & 0.1256 & 0.3487 & 0.3206 & 2.49 \\ \text { H11 } & 0.2205 & 0.7447 & 0.3928 & 2.48 \\ \text { H12 } & 0.2829 & 0.7392 & 0.5232 & 2.93 \\ \text { H13 } & 0.3365 & 0.6019 & 0.6411 & 2.80 \\ \text { H14 } & 0.3182 & 0.4718 & 0.6291 & 2.54 \\ \text { H19 } & -0.1068 & 0.6065 & 0.2336 & 3.00 \\ \text { H20 } & -0.1292 & 0.6073 & 0.0988 & 3.51 \\ \text { H21 } & 0.1144 & 0.5358 & 0.0371 & 3.69 \\ \text { H22 } & 0.3840 & 0.4644 & 0.1096 & 3.26 \\ \text { H23 } & 0.4088 & 0.4640 & 0.2429 & 2.69 \\ \text { H25 } & 0.6031 & 0.3318 & -0.0911 & 3.06 \\ \text { H26 } & 0.4155 & 0.4006 & -0.0081 & 3.27 \\ \text { H27 } & 0.2912 & 0.3158 & 0.1300 & 3.02 \\ \text { H28 } & 0.3590 & 0.1616 & 0.1889 & 2.54 \\ \text { H34 } & 0.8053 & -0.2341 & 0.1017 & 2.44 \\ \text { H35 } & 0.9662 & -0.2254 & -0.0272 & 3.05 \\ \text { H36 } & 1.0061 & -0.0858 & -0.1383 & 3.14 \\ \text { H37 } & 0.8804 & 0.0420 & -0.1212 & 2.78 \\ \text { H42 } & 0.6500 & 0.0394 & 0.2707 & 2.90 \\ \text { H43 } & 0.5015 & 0.0408 & 0.4001 & 3.70 \\ \text { H44 } & 0.2374 & -0.0271 & 0.4600 & 3.46 \\ \text { H45 } & 0.1151 & -0.0956 & 0.3901 & 3.43 \\ \text { H4 } & 0.2664 & -0.0991 & 0.2601 & 2.94 \\ \text { H3 } & 0.7135 & 0.2503 & 0.1709 & 4.45\end{array}$


Table 3. Anisotropic displacement parameters

\begin{tabular}{|c|c|c|c|c|c|c|}
\hline atom & $U_{11}$ & $\mathrm{U}_{22}$ & $U_{33}$ & $U_{12}$ & $U_{13}$ & $U_{23}$ \\
\hline $\mathrm{Cl} 1$ & $0.0342(8)$ & $0.0507(9)$ & $0.0487(9)$ & $-0.0106(6)$ & $0.0089(6)$ & $-0.0289(7)$ \\
\hline $\mathrm{Cl} 2$ & $0.0353(9)$ & $0.090(2)$ & $0.088(2)$ & $0.0022(8)$ & $-0.0093(8)$ & $-0.069(1)$ \\
\hline $\mathrm{Cl} 3$ & $0.057(1)$ & $0.087(2)$ & $0.053(1)$ & $-0.0375(9)$ & $0.0102(8)$ & $-0.0270(9)$ \\
\hline N1 & $0.023(2)$ & $0.028(2)$ & $0.024(3)$ & $-0.006(2)$ & $-0.001(2)$ & $-0.010(2)$ \\
\hline N2 & $0.039(3)$ & $0.031(3)$ & $0.029(3)$ & $-0.010(2)$ & $-0.001(2)$ & $-0.010(2)$ \\
\hline N3 & $0.028(3)$ & $0.030(3)$ & $0.020(3)$ & $-0.011(2)$ & $-0.002(2)$ & $-0.005(2)$ \\
\hline N4 & $0.041(3)$ & $0.032(3)$ & $0.023(3)$ & $-0.002(2)$ & $-0.001(2)$ & $-0.006(2)$ \\
\hline C1 & $0.017(3)$ & $0.030(3)$ & $0.026(3)$ & $-0.005(2)$ & $-0.001(2)$ & $-0.009(2)$ \\
\hline $\mathrm{C} 2$ & $0.025(3)$ & $0.030(3)$ & $0.026(3)$ & $-0.006(3)$ & $-0.001(2)$ & $-0.007(2)$ \\
\hline C3 & $0.036(3)$ & $0.023(3)$ & $0.040(4)$ & $-0.004(3)$ & $-0.002(3)$ & $-0.014(3)$ \\
\hline $\mathrm{C} 4$ & $0.026(3)$ & $0.037(3)$ & $0.033(3)$ & $-0.011(3)$ & $0.000(2)$ & $-0.019(3)$ \\
\hline C5 & $0.021(3)$ & $0.029(3)$ & $0.027(3)$ & $-0.007(2)$ & $0.000(2)$ & $-0.009(2)$ \\
\hline C6 & $0.016(3)$ & $0.025(3)$ & $0.022(3)$ & $-0.001(2)$ & $-0.001(2)$ & $-0.006(2)$ \\
\hline $\mathrm{C} 7$ & $0.017(3)$ & $0.026(3)$ & $0.023(3)$ & $-0.005(2)$ & $0.001(2)$ & $-0.011(2)$ \\
\hline C8 & $0.017(3)$ & $0.024(3)$ & $0.022(3)$ & $-0.002(2)$ & $-0.003(2)$ & $-0.007(2)$ \\
\hline C9 & $0.027(3)$ & $0.023(3)$ & $0.017(3)$ & $-0.005(2)$ & $-0.002(2)$ & $-0.004(2)$ \\
\hline C10 & $0.019(3)$ & $0.024(3)$ & $0.019(3)$ & $-0.009(2)$ & $0.002(2)$ & $-0.008(2)$ \\
\hline C11 & $0.023(3)$ & $0.026(3)$ & $0.026(3)$ & $-0.005(2)$ & $-0.000(2)$ & $-0.007(2)$ \\
\hline C12 & $0.024(3)$ & $0.036(3)$ & $0.042(4)$ & $-0.010(3)$ & $0.001(2)$ & $-0.023(3)$ \\
\hline C13 & $0.027(3)$ & $0.039(3)$ & $0.025(3)$ & $-0.004(3)$ & $-0.004(2)$ & $-0.014(3)$ \\
\hline C14 & $0.016(3)$ & $0.040(3)$ & $0.021(3)$ & $-0.005(2)$ & $-0.000(2)$ & $-0.010(2)$ \\
\hline C15 & $0.022(3)$ & $0.023(3)$ & $0.018(3)$ & $-0.004(2)$ & $0.003(2)$ & $-0.005(2)$ \\
\hline C16 & $0.015(3)$ & $0.022(3)$ & $0.016(3)$ & $-0.004(2)$ & $0.001(2)$ & $-0.004(2)$ \\
\hline C17 & $0.023(3)$ & $0.028(3)$ & $0.030(3)$ & $-0.011(2)$ & $-0.000(2)$ & $-0.014(3)$ \\
\hline C18 & $0.036(3)$ & $0.019(3)$ & $0.020(3)$ & $-0.006(2)$ & $-0.001(2)$ & $-0.004(2)$ \\
\hline C19 & $0.035(3)$ & $0.028(3)$ & $0.031(3)$ & $-0.002(3)$ & $-0.005(3)$ & $-0.012(3)$ \\
\hline C2O & $0.050(4)$ & $0.033(3)$ & $0.030(3)$ & $-0.011(3)$ & $-0.015(3)$ & $-0.009(3)$ \\
\hline $\mathrm{C} 21$ & $0.065(4)$ & $0.033(3)$ & $0.025(3)$ & $-0.017(3)$ & $-0.003(3)$ & $-0.014(3)$ \\
\hline C22 & $0.045(4)$ & $0.027(3)$ & $0.034(3)$ & $-0.011(3)$ & $0.007(3)$ & $-0.016(3)$ \\
\hline
\end{tabular}




$\begin{array}{lllllll}\text { C23 } & 0.036(3) & 0.024(3) & 0.024(3) & -0.006(3) & -0.000(2) & -0.008(2) \\ \text { C24 } & 0.023(3) & 0.034(3) & 0.029(3) & -0.009(3) & -0.005(2) & -0.011(3) \\ \text { C25 } & 0.039(3) & 0.026(3) & 0.027(3) & -0.013(3) & -0.002(3) & -0.004(2) \\ \text { C26 } & 0.032(3) & 0.031(3) & 0.042(4) & -0.011(3) & -0.008(3) & -0.011(3) \\ \text { C27 } & 0.031(3) & 0.031(3) & 0.031(3) & -0.004(3) & -0.003(2) & -0.011(3) \\ \text { C28 } & 0.026(3) & 0.033(3) & 0.026(3) & -0.011(3) & -0.005(2) & -0.012(2) \\ \text { C29 } & 0.020(3) & 0.027(3) & 0.025(3) & -0.009(2) & -0.004(2) & -0.008(2) \\ \text { C30 } & 0.022(3) & 0.029(3) & 0.024(3) & -0.011(2) & -0.002(2) & -0.010(2)\end{array}$


Table 3. Anisotropic displacement parameters (continued)

$\begin{array}{llllllc}\text { atom } & U_{11} & U_{22} & U_{33} & U_{12} & U_{13} & U_{23} \\ \text { C31 } & 0.021(3) & 0.033(3) & 0.020(3) & -0.009(2) & -0.004(2) & -0.011(2) \\ \text { C32 } & 0.023(3) & 0.027(3) & 0.023(3) & -0.006(2) & -0.005(2) & -0.010(2) \\ \text { C33 } & 0.022(3) & 0.036(3) & 0.023(3) & -0.010(3) & -0.005(2) & -0.012(2) \\ \text { C34 } & 0.025(3) & 0.027(3) & 0.030(3) & -0.006(2) & -0.006(2) & -0.014(2) \\ \text { C35 } & 0.030(3) & 0.040(3) & 0.033(3) & -0.006(3) & -0.004(2) & -0.020(3) \\ \text { C36 } & 0.032(3) & 0.046(3) & 0.028(3) & -0.012(3) & 0.001(2) & -0.020(3) \\ \text { C37 } & 0.027(3) & 0.039(3) & 0.024(3) & -0.014(3) & -0.001(2) & -0.012(2) \\ \text { C38 } & 0.022(3) & 0.033(3) & 0.021(3) & -0.009(2) & -0.003(2) & -0.011(2) \\ \text { C39 } & 0.013(3) & 0.031(3) & 0.027(3) & -0.008(2) & -0.003(2) & -0.011(2) \\ \text { C40 } & 0.027(3) & 0.033(3) & 0.028(3) & -0.006(3) & -0.005(2) & -0.015(3) \\ \text { C41 } & 0.034(3) & 0.025(3) & 0.020(3) & -0.006(3) & -0.003(2) & -0.006(2) \\ \text { C42 } & 0.031(3) & 0.030(3) & 0.028(3) & -0.008(3) & -0.003(2) & -0.008(2) \\ \text { C43 } & 0.050(4) & 0.037(3) & 0.035(4) & -0.003(3) & -0.004(3) & -0.020(3) \\ \text { C44 } & 0.050(4) & 0.031(3) & 0.027(3) & -0.004(3) & 0.005(3) & -0.014(3) \\ \text { C45 } & 0.029(3) & 0.036(3) & 0.043(4) & -0.010(3) & 0.005(3) & -0.016(3) \\ \text { C46 } & 0.035(3) & 0.024(3) & 0.032(3) & -0.004(3) & -0.001(3) & -0.010(2) \\ \text { C47 } & 0.037(4) & 0.074(4) & 0.050(4) & -0.022(3) & 0.006(3) & -0.041(4)\end{array}$

The general temperature factor expression: $\exp \left(-2 \pi^{2}\left(a^{\star 2} U_{11} h^{2}+b^{\star 2} U_{22} k^{2}+\left.c^{\star 2} U_{33}\right|^{2}+\right.\right.$ $\left.\left.2 a * b * U_{12} h k+2 a * c^{*} U_{13} h l+2 b * c^{*} U_{23} k l\right)\right)$ 
Table 4. Fragment Analysis

fragment: 1

$\mathrm{Cl}(1) \quad \mathrm{Cl}(2) \quad \mathrm{Cl}(3) \quad \mathrm{C}(47)$

fragment: 2

$\begin{array}{lllll}\mathrm{N}(1) & \mathrm{N}(2) & \mathrm{C}(1) & \mathrm{C}(2) & \mathrm{C}(3) \\ \mathrm{C}(4) & \mathrm{C}(5) & \mathrm{C}(6) & \mathrm{C}(7) & \mathrm{C}(8) \\ \mathrm{C}(9) & \mathrm{C}(10) & \mathrm{C}(11) & \mathrm{C}(12) & \mathrm{C}(13) \\ \mathrm{C}(14) & \mathrm{C}(15) & \mathrm{C}(16) & \mathrm{C}(17) & \mathrm{C}(18) \\ \mathrm{C}(19) & \mathrm{C}(20) & \mathrm{C}(21) & \mathrm{C}(22) & \mathrm{C}(23)\end{array}$

fragment: 3

$\begin{array}{lllll}\mathrm{N}(3) & \mathrm{N}(4) & \mathrm{C}(24) & \mathrm{C}(25) & \mathrm{C}(26) \\ \mathrm{C}(27) & \mathrm{C}(28) & \mathrm{C}(29) & \mathrm{C}(30) & \mathrm{C}(31) \\ \mathrm{C}(32) & \mathrm{C}(33) & \mathrm{C}(34) & \mathrm{C}(35) & \mathrm{C}(36) \\ \mathrm{C}(37) & \mathrm{C}(38) & \mathrm{C}(39) & \mathrm{C}(40) & \mathrm{C}(41) \\ \mathrm{C}(42) & \mathrm{C}(43) & \mathrm{C}(44) & \mathrm{C}(45) & \mathrm{C}(46)\end{array}$


Table 5. Bond lengths ( $\AA$ )

\begin{tabular}{|c|c|c|c|c|c|}
\hline atom & atom & distance & atom & atom & distance \\
\hline $\mathrm{Cl} 1$ & C47 & $1.771(6)$ & $\mathrm{Cl} 2$ & $\mathrm{C} 47$ & 1.801(6) \\
\hline $\mathrm{Cl} 3$ & C47 & $1.774(7)$ & N1 & $\mathrm{C} 1$ & 1.391(8) \\
\hline N1 & C16 & $1.382(5)$ & N2 & C17 & $1.169(5)$ \\
\hline N3 & $\mathrm{C} 24$ & $1.400(7)$ & N3 & C39 & $1.376(6)$ \\
\hline N4 & C40 & $1.160(6)$ & $\mathrm{C} 1$ & $\mathrm{C} 2$ & $1.406(7)$ \\
\hline C1 & C6 & $1.426(6)$ & C2 & C3 & $1.389(9)$ \\
\hline C3 & $\mathrm{C} 4$ & $1.429(7)$ & $\mathrm{C} 4$ & C5 & $1.381(7)$ \\
\hline C5 & C6 & $1.408(9)$ & $\mathrm{C} 6$ & $\mathrm{C} 7$ & $1.458(7)$ \\
\hline $\mathrm{C7}$ & $\mathrm{C} 8$ & $1.438(5)$ & $\mathrm{C} 7$ & C16 & $1.402(8)$ \\
\hline C8 & $\mathrm{Cg}$ & $1.395(8)$ & $\mathrm{C} 8$ & $\mathrm{C} 18$ & $1.488(8)$ \\
\hline C9 & C10 & $1.443(8)$ & C9 & C17 & $1.451(6)$ \\
\hline C10 & C11 & $1.426(8)$ & C10 & C15 & $1.436(5)$ \\
\hline C11 & C12 & $1.381(8)$ & $\mathrm{C} 12$ & $\mathrm{C} 13$ & $1.409(6)$ \\
\hline C13 & C14 & $1.384(9)$ & C14 & C15 & $1.410(8)$ \\
\hline C15 & C16 & $1.422(8)$ & C18 & C19 & $1.418(7)$ \\
\hline C18 & $\mathrm{C} 23$ & $1.421(7)$ & C19 & $\mathrm{C} 20$ & $1.400(9)$ \\
\hline C20 & $\mathrm{C} 21$ & $1.409(8)$ & $\mathrm{C} 21$ & $\mathrm{C} 22$ & $1.411(8)$ \\
\hline $\mathrm{C} 22$ & $\mathrm{C} 23$ & $1.385(8)$ & C24 & $\mathrm{C} 25$ & $1.399(7)$ \\
\hline C24 & $\mathrm{C} 29$ & $1.419(6)$ & $\mathrm{C} 25$ & $\mathrm{C} 26$ & $1.397(8)$ \\
\hline $\mathrm{C} 26$ & $\mathrm{C} 27$ & $1.414(7)$ & $\mathrm{C} 27$ & $\mathrm{C} 28$ & $1.392(7)$ \\
\hline C28 & C29 & $1.417(8)$ & $\mathrm{C} 29$ & C30 & $1.464(7)$ \\
\hline C30 & C31 & $1.422(6)$ & C30 & C39 & $1.412(7)$ \\
\hline C31 & C32 & $1.408(8)$ & C31 & $\mathrm{C} 41$ & $1.501(7)$ \\
\hline C32 & C33 & $1.456(7)$ & C32 & $\mathrm{C} 40$ & $1.436(6)$ \\
\hline C33 & C34 & $1.430(8)$ & C33 & C38 & $1.438(6)$ \\
\hline C34 & C35 & $1.380(7)$ & C35 & C36 & $1.413(7)$ \\
\hline C36 & C37 & $1.370(8)$ & $\mathrm{C} 37$ & C38 & $1.417(7)$ \\
\hline C38 & C39 & $1.420(8)$ & C41 & C42 & $1.409(9)$ \\
\hline C41 & C46 & $1.413(7)$ & C42 & C43 & $1.399(8)$ \\
\hline
\end{tabular}


C43 C44 1.399(8)

$\begin{array}{lll}\text { C44 } & \text { C45 }\end{array}$

C45 C46 1.418(8) 
Table 6. Bond lengths involving hydrogens $(\AA)$

$\begin{array}{llllll}\text { atom } & \text { atom } & \text { distance } & \text { atom } & \text { atom } & \text { distance } \\ \mathrm{N} 1 & \mathrm{H} 1 & 0.860 & \mathrm{~N} 3 & \mathrm{H} 3 & 0.860 \\ \mathrm{C} 2 & \mathrm{H} 2 & 0.930 & \mathrm{C} 3 & \mathrm{H} 3 \mathrm{~A} & 0.930 \\ \mathrm{C} 4 & \mathrm{H} 4 & 0.930 & \mathrm{C} 5 & \mathrm{H} 5 & 0.930 \\ \mathrm{C} 11 & \mathrm{H} 11 & 0.930 & \mathrm{C} 12 & \mathrm{H} 12 & 0.930 \\ \mathrm{C} 13 & \mathrm{H} 13 & 0.930 & \mathrm{C} 14 & \mathrm{H} 14 & 0.930 \\ \mathrm{C} 19 & \mathrm{H} 19 & 0.930 & \mathrm{C} 20 & \mathrm{H} 20 & 0.930 \\ \mathrm{C} 21 & \mathrm{H} 21 & 0.930 & \mathrm{C} 22 & \mathrm{H} 22 & 0.930 \\ \mathrm{C} 23 & \mathrm{H} 23 & 0.930 & \mathrm{C} 25 & \mathrm{H} 25 & 0.930 \\ \mathrm{C} 26 & \mathrm{H} 26 & 0.930 & \mathrm{C} 27 & \mathrm{H} 27 & 0.930 \\ \mathrm{C} 28 & \mathrm{H} 28 & 0.930 & \mathrm{C} 34 & \mathrm{H} 34 & 0.930 \\ \mathrm{C} 35 & \mathrm{H} 35 & 0.930 & \mathrm{C} 36 & \mathrm{H} 36 & 0.930 \\ \mathrm{C} 37 & \mathrm{H} 37 & 0.930 & \mathrm{C} 42 & \mathrm{H} 42 & 0.930 \\ \mathrm{C} 43 & \mathrm{H} 43 & 0.930 & \mathrm{C} 44 & \mathrm{H} 44 & 0.930 \\ \mathrm{C} 45 & \mathrm{H} 45 & 0.930 & \mathrm{C} 46 & \mathrm{H} 46 & 0.930 \\ \mathrm{C} 47 & \mathrm{H} 47 & 0.980 & & & \end{array}$


Table 7. Bond angles ( $\left.{ }^{\circ}\right)$

\begin{tabular}{|c|c|c|c|c|c|c|}
\hline atom & atom & angle & atom & atom & atom & angle \\
\hline N1 & C16 & $109.8(4)$ & $\mathrm{C} 24$ & N3 & C39 & $109.3(4)$ \\
\hline $\mathrm{C} 1$ & $\mathrm{C} 2$ & $129.0(5)$ & $\mathrm{N} 1$ & $\mathrm{C} 1$ & $\mathrm{C} 6$ & $108.5(4)$ \\
\hline C1 & $\mathrm{C} 6$ & $122.5(6)$ & $\mathrm{C} 1$ & $\mathrm{C} 2$ & C3 & $117.9(5)$ \\
\hline C3 & C4 & $120.7(5)$ & C3 & C4 & C5 & $120.7(6)$ \\
\hline C5 & C6 & $120.3(5)$ & $\mathrm{C} 1$ & C6 & C5 & $117.9(4)$ \\
\hline C6 & $\mathrm{C} 7$ & $105.4(5)$ & $\mathrm{C} 5$ & $\mathrm{C} 6$ & $\mathrm{C} 7$ & $136.6(4)$ \\
\hline $\mathrm{C7}$ & $\mathrm{C} 8$ & $132.9(5)$ & $\mathrm{C} 6$ & $\mathrm{C7}$ & C16 & $107.9(4)$ \\
\hline $\mathrm{C7}$ & C16 & $119.1(5)$ & $\mathrm{C} 7$ & C8 & $\mathrm{C} 9$ & $117.0(5)$ \\
\hline C8 & C18 & $119.7(5)$ & $\mathrm{Cg}$ & $\mathrm{C} 8$ & C18 & $123.2(4)$ \\
\hline C9 & C10 & $124.3(4)$ & $\mathrm{C} 8$ & C9 & C17 & $118.5(5)$ \\
\hline C10 & C17 & $117.2(5)$ & $\mathrm{C} 9$ & C10 & C11 & $123.1(4)$ \\
\hline C10 & C15 & $118.5(5)$ & C11 & C10 & C15 & $118.4(5)$ \\
\hline C11 & $\mathrm{C} 12$ & $121.2(4)$ & C11 & C12 & $\mathrm{C} 13$ & $119.9(6)$ \\
\hline C13 & C14 & $120.3(6)$ & $\mathrm{C} 13$ & C14 & C15 & $121.3(4)$ \\
\hline C15 & C14 & $118.8(5)$ & C10 & C15 & C16 & $116.3(5)$ \\
\hline C15 & C16 & $124.9(4)$ & $\mathrm{N} 1$ & C16 & $\mathrm{C} 7$ & $108.4(5)$ \\
\hline C16 & C15 & $126.8(5)$ & $\mathrm{C} 7$ & C16 & C15 & $124.8(4)$ \\
\hline C17 & $\mathrm{C} 9$ & $177.7(7)$ & $\mathrm{C} 8$ & C18 & C19 & $122.0(5)$ \\
\hline C18 & $\mathrm{C} 23$ & $118.5(4)$ & C19 & C18 & $\mathrm{C} 23$ & $119.5(5)$ \\
\hline C19 & $\mathrm{C} 20$ & $119.6(5)$ & C19 & $\mathrm{C} 20$ & $\mathrm{C} 21$ & $120.3(5)$ \\
\hline C21 & $\mathrm{C} 22$ & $120.1(6)$ & $\mathrm{C} 21$ & $\mathrm{C} 22$ & $\mathrm{C} 23$ & $119.9(5)$ \\
\hline $\mathrm{C} 23$ & $\mathrm{C} 22$ & $120.5(5)$ & N3 & C24 & $\mathrm{C} 25$ & $128.7(4)$ \\
\hline C24 & C29 & $108.9(4)$ & $\mathrm{C} 25$ & C24 & C29 & $122.3(5)$ \\
\hline $\mathrm{C} 25$ & C26 & $118.0(4)$ & $\mathrm{C} 25$ & $\mathrm{C} 26$ & $\mathrm{C} 27$ & $121.0(5)$ \\
\hline $\mathrm{C} 27$ & C28 & $120.6(5)$ & $\mathrm{C} 27$ & $\mathrm{C} 28$ & $\mathrm{C} 29$ & $119.7(4)$ \\
\hline C29 & $\mathrm{C} 28$ & $118.4(4)$ & $\mathrm{C} 24$ & C29 & C30 & $105.6(5)$ \\
\hline C29 & C30 & $135.8(4)$ & $\mathrm{C} 29$ & C30 & C31 & $134.1(5)$ \\
\hline C30 & C39 & $107.2(4)$ & C31 & C30 & с39 & $118.7(5)$ \\
\hline
\end{tabular}




$\begin{array}{llllllll}\text { C30 } & \text { C31 } & \text { C32 } & 118.4(4) & \text { C30 } & \text { C31 } & \text { C41 } & 119.4(5) \\ \text { C32 } & \text { C31 } & \text { C41 } & 122.2(4) & \text { C31 } & \text { C32 } & \text { C33 } & 122.9(4) \\ \text { C31 } & \text { C32 } & \text { C40 } & 121.1(5) & \text { C33 } & \text { C32 } & \text { C40 } & 115.9(5) \\ \text { C32 } & \text { C33 } & \text { C34 } & 123.2(4) & \text { C32 } & \text { C33 } & \text { C38 } & 118.4(5) \\ \text { C34 } & \text { C33 } & \text { C38 } & 118.4(4) & \text { C33 } & \text { C34 } & \text { C35 } & 121.5(4) \\ \text { C34 } & \text { C35 } & \text { C36 } & 119.5(5) & \text { C35 } & \text { C36 } & \text { C37 } & 120.6(5) \\ \text { C36 } & \text { C37 } & \text { C38 } & 121.8(4) & \text { C33 } & \text { C38 } & \text { C37 } & 118.3(5) \\ \text { C33 } & \text { C38 } & \text { C39 } & 116.8(4) & \text { C37 } & \text { C38 } & \text { C39 } & 124.9(4) \\ \text { N3 } & \text { C39 } & \text { C30 } & 109.0(5) & \text { N3 } & \text { C39 } & \text { C38 } & 126.3(4)\end{array}$


Table 7. Bond angles $\left(^{0}\right)$ (continued)

$\begin{array}{llllllll}\text { atom } & \text { atom } & \text { atom } & \text { angle } & \text { atom } & \text { atom } & \text { atom } & \text { angle } \\ \text { C30 } & \text { C39 } & \text { C38 } & 124.7(4) & \text { N4 } & \text { C40 } & \text { C32 } & 176.7(6) \\ \text { C31 } & \text { C41 } & \text { C42 } & 118.5(5) & \text { C31 } & \text { C41 } & \text { C46 } & 121.9(6) \\ \text { C42 } & \text { C41 } & \text { C46 } & 119.6(5) & \text { C41 } & \text { C42 } & \text { C43 } & 120.1(5) \\ \text { C42 } & \text { C43 } & \text { C44 } & 120.4(6) & \text { C43 } & \text { C44 } & \text { C45 } & 120.6(5) \\ \text { C44 } & \text { C45 } & \text { C46 } & 119.3(5) & \text { C41 } & \text { C46 } & \text { C45 } & 120.1(6) \\ \text { Cl1 } & \text { C47 } & \text { Cl2 } & 110.9(3) & \text { Cl1 } & \text { C47 } & \text { Cl3 } & 110.6(4) \\ \text { Cl2 } & \text { C47 } & \text { Cl3 } & 110.4(4) & & & & \end{array}$


Table 8. Bond angles involving hydrogens ( $\left.{ }^{\circ}\right)$

$\begin{array}{llllllll}\text { atom } & \text { atom } & \text { atom } & \text { angle } & \text { atom } & \text { atom } & \text { atom } & \text { angle } \\ \text { C1 } & \text { N1 } & \mathrm{H} 1 & 125 & \mathrm{C} 16 & \mathrm{~N} 1 & \mathrm{H} 1 & 125 \\ \mathrm{C} 24 & \mathrm{~N} 3 & \mathrm{H} 3 & 125 & \mathrm{C} 39 & \mathrm{~N} 3 & \mathrm{H} 3 & 125 \\ \mathrm{C} 1 & \mathrm{C} 2 & \mathrm{H} 2 & 121 & \mathrm{C} 3 & \mathrm{C} 2 & \mathrm{H} 2 & 121 \\ \mathrm{C} 2 & \mathrm{C} 3 & \mathrm{H} 3 \mathrm{~A} & 120 & \mathrm{C} 4 & \mathrm{C} 3 & \mathrm{H} 3 \mathrm{~A} & 120 \\ \mathrm{C} 3 & \mathrm{C} 4 & \mathrm{H} 4 & 120 & \mathrm{C} 5 & \mathrm{C} 4 & \mathrm{H} 4 & 120 \\ \mathrm{C} 4 & \mathrm{C} 5 & \mathrm{H} 5 & 120 & \mathrm{C} 6 & \mathrm{C} 5 & \mathrm{H} 5 & 120 \\ \mathrm{C} 10 & \mathrm{C} 11 & \mathrm{H} 11 & 119 & \mathrm{C} 12 & \mathrm{C} 11 & \mathrm{H} 11 & 119 \\ \mathrm{C} 11 & \mathrm{C} 12 & \mathrm{H} 12 & 120 & \mathrm{C} 13 & \mathrm{C} 12 & \mathrm{H} 12 & 120 \\ \mathrm{C} 12 & \mathrm{C} 13 & \mathrm{H} 13 & 120 & \mathrm{C} 14 & \mathrm{C} 13 & \mathrm{H} 13 & 120 \\ \mathrm{C} 13 & \mathrm{C} 14 & \mathrm{H} 14 & 119 & \mathrm{C} 15 & \mathrm{C} 14 & \mathrm{H} 14 & 119 \\ \mathrm{C} 18 & \mathrm{C} 19 & \mathrm{H} 19 & 120 & \mathrm{C} 20 & \mathrm{C} 19 & \mathrm{H} 19 & 120 \\ \mathrm{C} 19 & \mathrm{C} 20 & \mathrm{H} 20 & 120 & \mathrm{C} 21 & \mathrm{C} 20 & \mathrm{H} 20 & 120 \\ \mathrm{C} 20 & \mathrm{C} 21 & \mathrm{H} 21 & 120 & \mathrm{C} 22 & \mathrm{C} 21 & \mathrm{H} 21 & 120 \\ \mathrm{C} 21 & \mathrm{C} 22 & \mathrm{H} 22 & 120 & \mathrm{C} 23 & \mathrm{C} 22 & \mathrm{H} 22 & 120 \\ \mathrm{C} 18 & \mathrm{C} 23 & \mathrm{H} 23 & 120 & \mathrm{C} 22 & \mathrm{C} 23 & \mathrm{H} 23 & 120 \\ \mathrm{C} 24 & \mathrm{C} 25 & \mathrm{H} 25 & 121 & \mathrm{C} 26 & \mathrm{C} 25 & \mathrm{H} 25 & 121 \\ \mathrm{C} 25 & \mathrm{C} 26 & \mathrm{H} 26 & 119 & \mathrm{C} 27 & \mathrm{C} 26 & \mathrm{H} 26 & 119 \\ \mathrm{C} 26 & \mathrm{C} 27 & \mathrm{H} 27 & 120 & \mathrm{C} 28 & \mathrm{C} 27 & \mathrm{H} 27 & 120 \\ \mathrm{C} 27 & \mathrm{C} 28 & \mathrm{H} 28 & 120 & \mathrm{C} 29 & \mathrm{C} 28 & \mathrm{H} 28 & 120 \\ \mathrm{C} 33 & \mathrm{C} 34 & \mathrm{H} 34 & 119 & \mathrm{C} 35 & \mathrm{C} 34 & \mathrm{H} 34 & 119 \\ \mathrm{C} 34 & \mathrm{C} 35 & \mathrm{H} 35 & 120 & \mathrm{C} 36 & \mathrm{C} 35 & \mathrm{H} 35 & 120 \\ \mathrm{C} 35 & \mathrm{C} 36 & \mathrm{H} 36 & 120 & \mathrm{C} 37 & \mathrm{C} 36 & \mathrm{H} 36 & 120 \\ \mathrm{C} 36 & \mathrm{C} 37 & \mathrm{H} 37 & 119 & \mathrm{C} 38 & \mathrm{C} 37 & \mathrm{H} 37 & 119 \\ \mathrm{C} 41 & \mathrm{C} 42 & \mathrm{H} 42 & 120 & \mathrm{C} 43 & \mathrm{C} 42 & \mathrm{H} 42 & 120 \\ \mathrm{C} 42 & \mathrm{C} 43 & \mathrm{H} 43 & 120 & \mathrm{C} 44 & \mathrm{C} 43 & \mathrm{H} 43 & 120 \\ \mathrm{C} 43 & \mathrm{C} 44 & \mathrm{H} 44 & 120 & \mathrm{C} 45 & \mathrm{C} 44 & \mathrm{H} 44 & 120 \\ \mathrm{C} 44 & \mathrm{C} 45 & \mathrm{H} 45 & 120 & \mathrm{C} 46 & \mathrm{C} 45 & \mathrm{H} 45 & 120 \\ \mathrm{C} 41 & \mathrm{C} 46 & \mathrm{H} 46 & 120 & \mathrm{C} 45 & \mathrm{C} 46 & \mathrm{H} 46 & 120 \\ & & & & \mathrm{~S} 36 & & & \\ & & & & & & & \end{array}$




$\begin{array}{llllllll}\mathrm{Cl} 1 & \mathrm{C} 47 & \mathrm{H} 47 & 108 & \mathrm{Cl} 2 & \mathrm{C} 47 & \mathrm{H} 47 & 108 \\ \mathrm{Cl} 3 & \mathrm{C} 47 & \mathrm{H} 47 & 108 & & & & \end{array}$


Table 9. Torsion Angles( $\left({ }^{\circ}\right)$

(Those having bond angles $>160$ or $<20$ degrees are excluded.)

atom1 atom2 atom3 atom4 angle

$\begin{array}{lllll}\mathrm{C} 1 & \mathrm{~N} 1 & \mathrm{C} 16 & \mathrm{C} 7 & 0.0(5)\end{array}$

C16 N1 $\quad$ C1 $\quad$ C2 $179.8(4)$

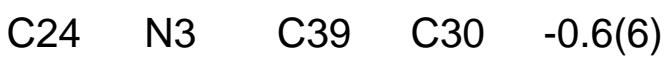

C39 N3 $\quad$ C24 $\quad$ C25 $-178.5(5)$

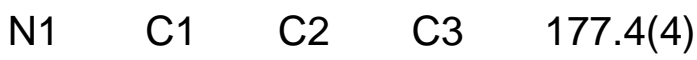

N1 $\quad \mathrm{C} 1 \quad \mathrm{C} 6 \quad \mathrm{C} 7 \quad 1.3(5)$

$\begin{array}{lllll}\mathrm{C} 2 & \mathrm{C} 1 & \mathrm{C} 6 & \mathrm{C} 7 & -179.3(4)\end{array}$

$\begin{array}{lllll}\mathrm{C} 1 & \mathrm{C} 2 & \mathrm{C} 3 & \mathrm{C} 4 & 0.0(7)\end{array}$

$\mathrm{C} 3 \quad \mathrm{C} 4 \quad \mathrm{C} 5 \quad \mathrm{C} 6 \quad 1.0(7)$

$\mathrm{C} 4 \quad \mathrm{C} 5 \quad \mathrm{C} 6 \quad \mathrm{C} 7 \quad-179.2(4)$

$\mathrm{C} 1 \quad \mathrm{C} 6 \quad \mathrm{C} 7 \quad \mathrm{C} 16 \quad-1.3(4)$

$\mathrm{C} 5 \quad \mathrm{C} 6 \quad \mathrm{C} 7 \quad \mathrm{C} 16 \quad 175.5(5)$

$\begin{array}{lllll}\mathrm{C} 6 & \mathrm{C} 7 & \mathrm{C} 8 & \mathrm{C} 18 & -5.2(7)\end{array}$

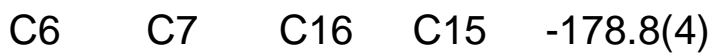

C8 $\quad \mathrm{C} 7 \quad \mathrm{C} 16 \quad \mathrm{C} 15 \quad 2.1(6)$

$\begin{array}{lllll}\mathrm{C} 16 & \mathrm{C} 7 & \mathrm{C} 8 & \mathrm{C} 18 & 173.7(4)\end{array}$

$\mathrm{C} 7 \quad \mathrm{C} 8 \quad \mathrm{C} 9 \quad \mathrm{C} 17 \quad 179.6(4)$

$\mathrm{C} 7 \quad \mathrm{C} 8 \quad \mathrm{C} 18 \quad \mathrm{C} 23 \quad-61.4(6)$

$\begin{array}{lllll}\mathrm{C} 9 & \mathrm{C} 8 & \mathrm{C} 18 & \mathrm{C} 23 & 114.6(5)\end{array}$

$\mathrm{C} 18 \quad \mathrm{C} 8 \quad \mathrm{C} 9 \quad \mathrm{C} 17 \quad 3.5(6)$

$\begin{array}{lllll}\mathrm{C} 8 & \mathrm{C} 9 & \mathrm{C} 10 & \mathrm{C} 15 & 0.3(6)\end{array}$

C17 C9 C10 C15 $-177.9(4)$

C9 $\quad \mathrm{C} 10 \quad \mathrm{C} 15 \quad \mathrm{C} 14 \quad 178.4(4)$

C11 C10 C15 C14 0.4(6)

C15 C10 C11 C12 $-1.1(6)$

C11 C12 C13 C14 $-0.9(7)$

C13 C14 C15 C10 $0.1(6)$ atom1 atom2 atom3 atom4 angle

C1 N1 C16 C15 179.6(4)

C16 N1 C1 C6 $-0.9(5)$

C24 N3 $\quad$ C39 $\quad$ C38 $178.3(5)$

C39 N3 $\quad$ C24 $\quad$ C29 $\quad-0.3(6)$

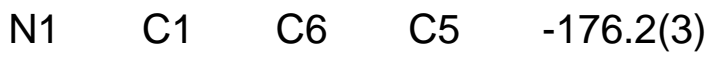

C2 $\quad$ C1 6 C6 $55 \quad 3.2(6)$

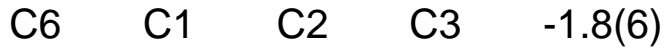

$\begin{array}{lllll}\mathrm{C} 2 & \mathrm{C} 3 & \mathrm{C} 4 & \mathrm{C} 5 & 0.4(7)\end{array}$

$\mathrm{C} 4 \quad \mathrm{C} 5 \quad \mathrm{C} 6 \quad \mathrm{C} 1 \quad-2.7(6)$

$\begin{array}{lllll}\mathrm{C} 1 & \mathrm{C} 6 & \mathrm{C} 7 & \mathrm{C} 8 & 177.7(4)\end{array}$

$\begin{array}{lllll}\mathrm{C} 5 & \mathrm{C} 6 & \mathrm{C} 7 & \mathrm{C} 8 & -5.5(8)\end{array}$

$\begin{array}{lllll}\mathrm{C} 6 & \mathrm{C} 7 & \mathrm{C} 8 & \mathrm{C} 9 & 178.6(4)\end{array}$

$\begin{array}{lllll}\mathrm{C} 6 & \mathrm{C} 7 & \mathrm{C} 16 & \mathrm{~N} 1 & 0.8(5)\end{array}$

$\begin{array}{lllll}\mathrm{C} 8 & \mathrm{C} 7 & \mathrm{C} 16 & \mathrm{~N} 1 & -178.4(4)\end{array}$

$\begin{array}{lllll}\mathrm{C} 16 & \mathrm{C} 7 & \mathrm{C} 8 & \mathrm{C} 9 & -2.5(6)\end{array}$

$\mathrm{C} 7 \quad \mathrm{C} 8 \quad \mathrm{C} 9 \quad \mathrm{C} 10 \quad 1.4(6)$

$\mathrm{C} 7 \quad \mathrm{C} 8 \quad \mathrm{C} 18 \quad \mathrm{C} 19 \quad 118.6(4)$

C9 $\quad \mathrm{C} 8 \quad \mathrm{C} 18 \quad \mathrm{C} 19 \quad-65.4(6)$

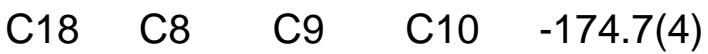

C8 $\quad$ C9 $\quad$ C10 C11 $178.2(4)$

C17 C9 C10 C11 0.0(6)

C9 $\quad$ C10 C11 C12 $-179.0(4)$

C9 C10 C15 C16 $-0.9(6)$

C11 C10 C15 C16 $-178.9(4)$

C10 C11 C12 C13 $1.4(7)$

C12 $\mathrm{C} 13 \quad \mathrm{C} 14 \quad \mathrm{C} 15 \quad 0.2(7)$

C13 C14 C15 C16 179.3(4) 


$\begin{array}{llllllllll}\text { C10 } & \text { C15 } & \text { C16 } & \text { N1 } & -179.8(4) & \text { C10 } & \text { C15 } & \text { C16 } & \text { C7 } & -0.3(6) \\ \text { C14 } & \text { C15 } & \text { C16 } & \text { N1 } & 1.0(7) & \text { C14 } & \text { C15 } & \text { C16 } & \text { C7 } & -179.5(4) \\ \text { C8 } & \text { C18 } & \text { C19 } & \text { C20 } & 179.2(4) & \text { C8 } & \text { C18 } & \text { C23 } & \text { C22 } & -179.5(4) \\ \text { C19 } & \text { C18 } & \text { C23 } & \text { C22 } & 0.5(7) & \text { C23 } & \text { C18 } & \text { C19 } & \text { C20 } & -0.8(7) \\ \text { C18 } & \text { C19 } & \text { C20 } & \text { C21 } & 0.7(7) & \text { C19 } & \text { C20 } & \text { C21 } & \text { C22 } & -0.3(8) \\ \text { C20 } & \text { C21 } & \text { C22 } & \text { C23 } & 0.1(8) & \text { C21 } & \text { C22 } & \text { C23 } & \text { C18 } & -0.2(7) \\ \text { N3 } & \text { C24 } & \text { C25 } & \text { C26 } & 175.6(5) & \text { N3 } & \text { C24 } & \text { C29 } & \text { C28 } & -175.5(4) \\ \text { N3 } & \text { C24 } & \text { C29 } & \text { C30 } & 1.0(6) & \text { C25 } & \text { C24 } & \text { C29 } & \text { C28 } & 2.8(8) \\ \text { C25 } & \text { C24 } & \text { C29 } & \text { C30 } & 179.4(5) & \text { C29 } & \text { C24 } & \text { C25 } & \text { C26 } & -2.3(8) \\ \text { C24 } & \text { C25 } & \text { C26 } & \text { C27 } & 0.3(8) & \text { C25 } & \text { C26 } & \text { C27 } & \text { C28 } & 1.1(9)\end{array}$


Table 9. Torsion angles $\left({ }^{\circ}\right)$ (continued)

atom1 atom2 atom3 atom4 angle

$\begin{array}{lllll}\mathrm{C} 26 & \mathrm{C} 27 & \mathrm{C} 28 & \mathrm{C} 29 & -0.7(8)\end{array}$

$\begin{array}{lllll}\mathrm{C} 27 & \mathrm{C} 28 & \mathrm{C} 29 & \mathrm{C} 30 & -176.5(5)\end{array}$

C24 C29 C30 C39 $-1.4(6)$

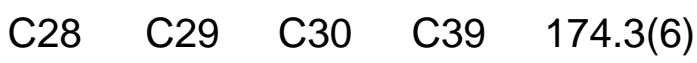

C29 C30 C31 C41 $-4.1(9)$

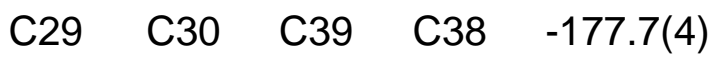

$\begin{array}{lllll}\text { C31 } & \text { C30 } & \text { C39 } & \text { C38 } & 1.6(8)\end{array}$

C39 C30 C31 C41 $176.9(4)$

C30 C31 C32 C40 $-180.0(4)$

C30 C31 C41 C46 120.9(5)

C32 C31 C41 C46 $-59.2(6)$

$\begin{array}{lllll}\mathrm{C} 41 & \mathrm{C} 31 & \mathrm{C} 32 & \mathrm{C} 40 & 0.2(7)\end{array}$

$\begin{array}{lllll}\text { C31 } & \text { C32 } & \text { C33 } & \text { C38 } & -1.1(7)\end{array}$

$\begin{array}{lllll}\mathrm{C} 40 & \mathrm{C} 32 & \mathrm{C} 33 & \mathrm{C} 38 & -178.5(4)\end{array}$

C32 $\quad$ C33 $\quad$ C38 $\quad$ C37 $178.8(4)$

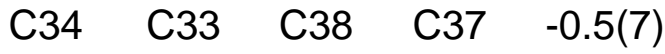

$\begin{array}{lllll}\mathrm{C} 38 & \mathrm{C} 33 & \mathrm{C} 34 & \mathrm{C} 35 & 0.3(7)\end{array}$

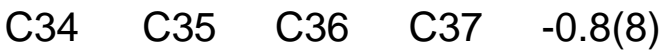

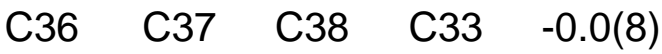

C33 $\quad$ C38 $\quad$ C39 N3 $\quad-178.6(4)$

$\begin{array}{lllll}\mathrm{C} 37 & \mathrm{C} 38 & \mathrm{C} 39 & \mathrm{~N} 3 & 2.3(8)\end{array}$

C31 C41 C42 C43 177.8(3)

C42 C41 C46 C45 $1.7(6)$

C41 C42 C43 C44 $0.6(6)$

$\begin{array}{llllll}\mathrm{C} 43 & \mathrm{C} 44 & \mathrm{C} 45 & \mathrm{C} 46 & 0.8(7)\end{array}$ atom1 atom2 atom3 atom4 angle

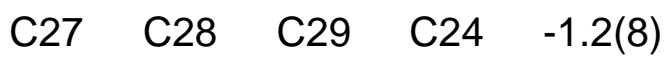

C24 C29 C30 C31 $179.6(5)$

C28 C29 C30 C31 $-4.8(11)$

C29 C30 C31 C32 176.0(5)

$\begin{array}{lllll}\mathrm{C} 29 & \mathrm{C} 30 & \mathrm{C} 39 & \mathrm{~N} 3 & 1.2(6)\end{array}$

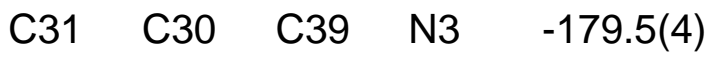

$\begin{array}{lllll}\text { C39 } & \text { C30 } & \text { C31 } & \text { C32 } & -3.0(7)\end{array}$

$\begin{array}{lllll}\text { C30 } & \text { C31 } & \text { C32 } & \text { C33 } & 2.8(7)\end{array}$

C30 C31 C41 C42 $-58.1(6)$

C32 C31 C41 C42 121.8(5)

C41 C31 C32 C33 $-177.0(4)$

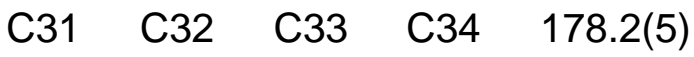

$\begin{array}{lllll}\mathrm{C} 40 & \mathrm{C} 32 & \mathrm{C} 33 & \mathrm{C} 34 & 0.8(7)\end{array}$

$\begin{array}{lllll}\mathrm{C} 32 & \mathrm{C} 33 & \mathrm{C} 34 & \mathrm{C} 35 & -179.0(5)\end{array}$

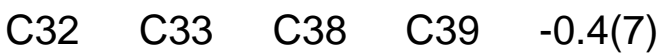

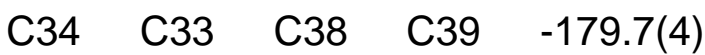

$\begin{array}{lllll}\mathrm{C} 33 & \mathrm{C} 34 & \mathrm{C} 35 & \mathrm{C} 36 & 0.3(8)\end{array}$

$\begin{array}{lllll}\mathrm{C} 35 & \mathrm{C} 36 & \mathrm{C} 37 & \mathrm{C} 38 & 0.7(8)\end{array}$

$\begin{array}{lllll}\text { C36 } & \text { C37 } & \text { C38 } & \text { C39 } & 179.2(5)\end{array}$

$\begin{array}{lllll}\text { C33 } & \text { C38 } & \text { C39 } & \text { C30 } & 0.1(7)\end{array}$

C37 C38 C39 C30 $\quad-179.1(5)$

C31 C41 C46 C45 $-177.3(3)$

C46 C41 C42 C43 $-1.2(6)$

$\mathrm{C} 42 \quad \mathrm{C} 43 \quad \mathrm{C} 44 \quad \mathrm{C} 45 \quad-0.3(7)$

C44 C45 C46 C41 $-1.4(6)$ 
Table 10. Possible hydrogen bonds

$\begin{array}{lllllll}\text { Donor } & \mathrm{H} & \text { Acceptor } & \text { D...A } & \text { D-H } & \text { H...A } & \text { D-H...A } \\ \text { N1 } & \mathrm{H} 1 & \mathrm{~N}^{1} & 2.954(6) & 0.86 & 2.13 & 160.42 \\ \mathrm{~N} 3 & \mathrm{H} 3 & \mathrm{~N}^{2} & 2.976(6) & 0.86 & 2.13 & 169.76\end{array}$

Symmetry Operators:
(1) $-X+1,-Y,-Z+1$
(2) $-X+1,-Y+1,-Z$ 
Table 11. Intramolecular contacts less than $3.60 \AA$

\begin{tabular}{|c|c|c|c|c|c|}
\hline atom & atom & distance & atom & atom & distance \\
\hline N1 & C5 & $3.599(8)$ & N1 & C14 & $3.057(8)$ \\
\hline N2 & $\mathrm{C} 8$ & $3.523(6)$ & N2 & C10 & $3.504(6)$ \\
\hline N2 & C11 & $3.568(7)$ & N3 & C37 & $3.046(7)$ \\
\hline N4 & C31 & $3.557(6)$ & N4 & C33 & $3.462(5)$ \\
\hline N4 & C34 & $3.513(6)$ & $\mathrm{C} 1$ & $\mathrm{C} 4$ & $2.775(9)$ \\
\hline $\mathrm{C} 2$ & C5 & $2.842(7)$ & C3 & C6 & $2.821(7)$ \\
\hline C5 & $\mathrm{C} 8$ & $3.463(7)$ & C5 & C18 & $3.383(6)$ \\
\hline C5 & $\mathrm{C} 23$ & $3.308(6)$ & C6 & C18 & $3.179(5)$ \\
\hline C6 & $\mathrm{C} 23$ & $3.403(6)$ & $\mathrm{C} 7$ & C10 & $2.861(8)$ \\
\hline$C 7$ & $\mathrm{C} 23$ & $3.149(7)$ & C8 & C15 & $2.915(8)$ \\
\hline C9 & C16 & $2.753(5)$ & $\mathrm{C9}$ & C19 & $3.264(9)$ \\
\hline $\mathrm{C} 9$ & $\mathrm{C} 23$ & $3.566(8)$ & C10 & C13 & $2.823(8)$ \\
\hline C11 & C14 & $2.796(6)$ & C11 & C17 & $2.884(8)$ \\
\hline C12 & C15 & $2.827(8)$ & C17 & C18 & $2.903(8)$ \\
\hline C17 & C19 & $3.190(9)$ & C18 & $\mathrm{C} 21$ & $2.811(8)$ \\
\hline C19 & $\mathrm{C} 22$ & $2.824(8)$ & C20 & $\mathrm{C} 23$ & $2.809(7)$ \\
\hline C24 & $\mathrm{C} 27$ & $2.782(8)$ & $\mathrm{C} 25$ & $\mathrm{C} 28$ & $2.842(6)$ \\
\hline $\mathrm{C} 26$ & $\mathrm{C} 29$ & $2.813(7)$ & $\mathrm{C} 28$ & C31 & $3.471(7)$ \\
\hline $\mathrm{C} 28$ & C41 & $3.389(6)$ & $\mathrm{C} 28$ & C42 & $3.227(6)$ \\
\hline C29 & C41 & $3.190(6)$ & C29 & C42 & $3.356(6)$ \\
\hline C30 & C33 & $2.876(8)$ & C30 & C42 & $3.113(7)$ \\
\hline C31 & C38 & $2.906(7)$ & C32 & C39 & $2.771(6)$ \\
\hline C32 & C46 & $3.223(7)$ & C33 & C36 & $2.822(7)$ \\
\hline C34 & C37 & $2.789(6)$ & C34 & C40 & $2.869(7)$ \\
\hline C35 & C38 & $2.836(8)$ & C40 & C41 & $2.950(8)$ \\
\hline C40 & C46 & $3.171(7)$ & C41 & C44 & $2.813(8)$ \\
\hline C42 & C45 & $2.820(8)$ & C43 & C46 & $2.808(10)$ \\
\hline
\end{tabular}


Table 12. Intramolecular contacts less than $3.60 \AA$ involving hydrogens

\begin{tabular}{|c|c|c|c|c|c|}
\hline atom & atom & distance & atom & atom & distance \\
\hline N1 & $\mathrm{H} 2$ & 2.776 & N1 & $\mathrm{H} 14$ & 2.797 \\
\hline N2 & $\mathrm{H} 11$ & 2.964 & N2 & $\mathrm{H} 19$ & 3.442 \\
\hline N3 & $\mathrm{H} 25$ & 2.770 & N3 & H37 & 2.784 \\
\hline N4 & H34 & 2.904 & N4 & $\mathrm{H} 46$ & 3.342 \\
\hline C1 & $\mathrm{H} 3 \mathrm{~A}$ & 3.239 & $\mathrm{C} 1$ & H5 & 3.274 \\
\hline $\mathrm{C} 2$ & $\mathrm{H} 1$ & 2.797 & $\mathrm{C} 2$ & $\mathrm{H} 4$ & 3.280 \\
\hline C3 & H5 & 3.281 & C4 & $\mathrm{H} 2$ & 3.294 \\
\hline C5 & $\mathrm{H} 3 \mathrm{~A}$ & 3.273 & C5 & $\mathrm{H} 23$ & 3.233 \\
\hline C6 & $\mathrm{H} 1$ & 3.114 & C6 & $\mathrm{H} 2$ & 3.321 \\
\hline C6 & $\mathrm{H} 4$ & 3.257 & $\mathrm{C} 6$ & $\mathrm{H} 23$ & 3.182 \\
\hline $\mathrm{C7}$ & $\mathrm{H} 1$ & 3.084 & $\mathrm{C7}$ & $\mathrm{H} 5$ & 2.937 \\
\hline $\mathrm{C7}$ & $\mathrm{H} 23$ & 2.995 & $\mathrm{C} 8$ & $\mathrm{H} 5$ & 3.347 \\
\hline C8 & $\mathrm{H} 19$ & 2.713 & C8 & $\mathrm{H} 23$ & 2.639 \\
\hline $\mathrm{Cg}$ & $\mathrm{H} 11$ & 2.699 & $\mathrm{Cg}$ & $\mathrm{H} 19$ & 3.181 \\
\hline C10 & $\mathrm{H} 12$ & 3.284 & C10 & $\mathrm{H} 14$ & 3.291 \\
\hline C11 & $\mathrm{H} 13$ & 3.251 & C12 & $\mathrm{H} 14$ & 3.258 \\
\hline C13 & $\mathrm{H} 11$ & 3.253 & C14 & $\mathrm{H} 1$ & 2.893 \\
\hline C14 & $\mathrm{H} 12$ & 3.259 & C15 & $\mathrm{H} 1$ & 2.763 \\
\hline C15 & $\mathrm{H} 11$ & 3.299 & C15 & $\mathrm{H} 13$ & 3.271 \\
\hline C16 & $\mathrm{H} 14$ & 2.703 & C17 & $\mathrm{H} 11$ & 2.558 \\
\hline C17 & $\mathrm{H} 19$ & 3.044 & C18 & $\mathrm{H} 5$ & 2.883 \\
\hline C18 & $\mathrm{H} 2 \mathrm{O}$ & 3.275 & C18 & $\mathrm{H} 22$ & 3.276 \\
\hline C19 & H5 & 3.511 & C19 & $\mathrm{H} 21$ & 3.274 \\
\hline C19 & $\mathrm{H} 23$ & 3.290 & $\mathrm{C} 20$ & $\mathrm{H} 22$ & 3.282 \\
\hline C21 & H19 & 3.276 & C21 & $\mathrm{H} 23$ & 3.260 \\
\hline C22 & H5 & 3.290 & $\mathrm{C} 22$ & $\mathrm{H} 2 \mathrm{O}$ & 3.281 \\
\hline $\mathrm{C} 23$ & H5 & 2.752 & $\mathrm{C} 23$ & $\mathrm{H} 19$ & 3.294 \\
\hline $\mathrm{C} 23$ & $\mathrm{H} 21$ & 3.258 & $\mathrm{C} 24$ & $\mathrm{H} 26$ & 3.238 \\
\hline
\end{tabular}




$\begin{array}{llllll}\mathrm{C} 24 & \mathrm{H} 28 & 3.281 & \mathrm{C} 25 & \mathrm{H} 3 & 2.800 \\ \mathrm{C} 25 & \mathrm{H} 27 & 3.279 & \mathrm{C} 26 & \mathrm{H} 28 & 3.277 \\ \mathrm{C} 27 & \mathrm{H} 25 & 3.290 & \mathrm{C} 28 & \mathrm{H} 26 & 3.269 \\ \mathrm{C} 28 & \mathrm{H} 42 & 3.127 & \mathrm{C} 29 & \mathrm{H} 3 & 3.121 \\ \mathrm{C} 29 & \mathrm{H} 25 & 3.307 & \mathrm{C} 29 & \mathrm{H} 27 & 3.269 \\ \mathrm{C} 29 & \mathrm{H} 42 & 3.119 & \mathrm{C} 30 & \mathrm{H} 3 & 3.097 \\ \mathrm{C} 30 & \mathrm{H} 28 & 2.943 & \mathrm{C} 30 & \mathrm{H} 42 & 2.959 \\ \mathrm{C} 31 & \mathrm{H} 28 & 3.361 & \mathrm{C} 31 & \mathrm{H} 42 & 2.640 \\ \mathrm{C} 31 & \mathrm{H} 46 & 2.712 & \mathrm{C} 32 & \mathrm{H} 34 & 2.713\end{array}$


Table 12. Intramolecular contacts less than $3.60 \AA$ involving hydrogens (continued)

\begin{tabular}{|c|c|c|c|c|c|}
\hline atom & atom & distance & atom & atom & distance \\
\hline C32 & $\mathrm{H} 46$ & 3.085 & C33 & H35 & 3.290 \\
\hline C33 & H37 & 3.290 & C34 & $\mathrm{H} 36$ & 3.249 \\
\hline C35 & H37 & 3.253 & C36 & H34 & 3.251 \\
\hline C37 & $\mathrm{H} 3$ & 2.878 & C37 & H35 & 3.253 \\
\hline C38 & $\mathrm{H} 3$ & 2.751 & C38 & H34 & 3.302 \\
\hline C38 & H36 & 3.270 & C39 & H37 & 2.705 \\
\hline $\mathrm{C} 40$ & $\mathrm{H} 34$ & 2.544 & $\mathrm{C} 40$ & $\mathrm{H} 46$ & 2.930 \\
\hline C41 & $\mathrm{H} 28$ & 2.887 & C41 & $\mathrm{H} 43$ & 3.271 \\
\hline C41 & H45 & 3.293 & C42 & $\mathrm{H} 28$ & 2.632 \\
\hline C42 & $\mathrm{H} 44$ & 3.264 & C42 & $\mathrm{H} 46$ & 3.278 \\
\hline $\mathrm{C} 43$ & $\mathrm{H} 28$ & 3.141 & $\mathrm{C} 43$ & $\mathrm{H} 45$ & 3.272 \\
\hline C44 & $\mathrm{H} 42$ & 3.265 & C44 & $\mathrm{H} 46$ & 3.273 \\
\hline C45 & $\mathrm{H} 43$ & 3.269 & C46 & $\mathrm{H} 28$ & 3.585 \\
\hline C46 & $\mathrm{H} 42$ & 3.278 & C46 & $\mathrm{H} 44$ & 3.273 \\
\hline $\mathrm{H} 1$ & $\mathrm{H} 2$ & 2.760 & $\mathrm{H} 1$ & $\mathrm{H} 14$ & 2.387 \\
\hline $\mathrm{H} 2$ & $\mathrm{H} 3 \mathrm{~A}$ & 2.329 & $\mathrm{H} 3$ & $\mathrm{H} 25$ & 2.758 \\
\hline $\mathrm{H} 3$ & H37 & 2.369 & $\mathrm{H} 3 \mathrm{~A}$ & $\mathrm{H} 4$ & 2.349 \\
\hline $\mathrm{H} 4$ & $\mathrm{H} 5$ & 2.304 & H5 & $\mathrm{H} 23$ & 2.896 \\
\hline $\mathrm{H} 11$ & $\mathrm{H} 12$ & 2.303 & $\mathrm{H} 12$ & $\mathrm{H} 13$ & 2.338 \\
\hline $\mathrm{H} 13$ & $\mathrm{H} 14$ & 2.303 & $\mathrm{H} 19$ & $\mathrm{H} 2 \mathrm{O}$ & 2.331 \\
\hline $\mathrm{H} 2 \mathrm{O}$ & $\mathrm{H} 21$ & 2.336 & $\mathrm{H} 21$ & $\mathrm{H} 22$ & 2.341 \\
\hline $\mathrm{H} 22$ & $\mathrm{H} 23$ & 2.312 & $\mathrm{H} 25$ & $\mathrm{H} 26$ & 2.333 \\
\hline H26 & $\mathrm{H} 27$ & 2.333 & $\mathrm{H} 27$ & $\mathrm{H} 28$ & 2.321 \\
\hline $\mathrm{H} 28$ & $\mathrm{H} 42$ & 2.743 & $\mathrm{H} 28$ & $\mathrm{H} 43$ & 3.516 \\
\hline H34 & H35 & 2.303 & H35 & $\mathrm{H} 36$ & 2.343 \\
\hline H36 & H37 & 2.284 & $\mathrm{H} 42$ & $\mathrm{H} 43$ & 2.326 \\
\hline $\mathrm{H} 43$ & $\mathrm{H} 44$ & 2.322 & $\mathrm{H} 44$ & $\mathrm{H} 45$ & 2.333 \\
\hline $\mathrm{H} 45$ & H46 & 2.353 & & & \\
\hline
\end{tabular}


Table 13. Intermolecular contacts less than $3.60 \AA$

\begin{tabular}{|c|c|c|c|c|c|}
\hline atom & atom & distance & atom & atom & distance \\
\hline $\mathrm{Cl} 1$ & C4 & $3.290(5)$ & Cl1 & C5 & $3.290(5)$ \\
\hline $\mathrm{Cl} 3$ & $\mathrm{C} 21^{1}$ & $3.585(6)$ & N1 & $N 4^{2}$ & $2.954(6)$ \\
\hline N1 & $C 9^{3}$ & $3.568(6)$ & N1 & $\mathrm{C} 10^{3}$ & $3.555(6)$ \\
\hline N1 & $\mathrm{C} 11^{4}$ & $3.585(6)$ & N1 & C12 ${ }^{4}$ & $3.440(6)$ \\
\hline N2 & $N 3^{5}$ & $2.976(6)$ & N3 & $\mathrm{N} 2^{5}$ & $2.976(6)$ \\
\hline N3 & $\mathrm{C} 33^{6}$ & $3.572(7)$ & N3 & $\mathrm{C} 35^{7}$ & $3.381(8)$ \\
\hline N3 & $\mathrm{C} 36^{7}$ & $3.482(7)$ & N4 & $N 1^{2}$ & $2.954(6)$ \\
\hline N4 & $\mathrm{C} 17^{8}$ & $3.547(7)$ & $\mathrm{C} 1$ & $\mathrm{C} 11^{3}$ & $3.522(6)$ \\
\hline C1 & $\mathrm{C} 12^{4}$ & $3.500(7)$ & $\mathrm{C} 4$ & Cl1 & $3.290(5)$ \\
\hline $\mathrm{C} 4$ & $\mathrm{C} 12^{3}$ & $3.600(7)$ & C5 & $\mathrm{Cl} 1$ & $3.290(5)$ \\
\hline C5 & $\mathrm{C} 12^{3}$ & $3.445(7)$ & C5 & $\mathrm{C} 13^{3}$ & $3.513(6)$ \\
\hline C7 & $\mathrm{C} 13^{4}$ & $3.531(6)$ & C7 & $\mathrm{C} 15^{3}$ & $3.497(6)$ \\
\hline C9 & $N 1^{3}$ & $3.568(6)$ & C10 & $\mathrm{N} 1^{3}$ & $3.555(6)$ \\
\hline C11 & $\mathrm{N} 1^{4}$ & $3.585(6)$ & C11 & $\mathrm{C} 1^{3}$ & $3.522(6)$ \\
\hline C12 & $\mathrm{N} 1^{4}$ & $3.440(6)$ & C12 & $\mathrm{C} 1^{4}$ & $3.500(7)$ \\
\hline C12 & $\mathrm{C} 4^{3}$ & $3.600(7)$ & C12 & $C 5^{3}$ & $3.445(7)$ \\
\hline C13 & $C 5^{3}$ & $3.513(6)$ & C13 & $C 7^{4}$ & $3.531(6)$ \\
\hline C13 & C16 ${ }^{4}$ & $3.472(6)$ & C14 & $C 15^{4}$ & $3.500(6)$ \\
\hline C14 & C16 ${ }^{4}$ & $3.452(6)$ & C15 & $C 7^{3}$ & $3.497(6)$ \\
\hline C15 & C14 ${ }^{4}$ & $3.500(6)$ & C15 & $\mathrm{C} 15^{4}$ & $3.531(6)$ \\
\hline C16 & $\mathrm{C} 13^{4}$ & $3.472(6)$ & C16 & C14 ${ }^{4}$ & $3.452(6)$ \\
\hline C17 & $N 4^{9}$ & $3.547(7)$ & $\mathrm{C} 21$ & $\mathrm{Cl}^{10}$ & $3.585(6)$ \\
\hline $\mathrm{C} 22$ & $C 25^{5}$ & $3.532(7)$ & $\mathrm{C} 24$ & $\mathrm{C} 34^{6}$ & $3.460(8)$ \\
\hline $\mathrm{C} 25$ & $C 22^{5}$ & $3.532(7)$ & $\mathrm{C} 28$ & $\mathrm{C} 35^{6}$ & $3.452(9)$ \\
\hline C29 & $\mathrm{C} 34^{6}$ & $3.590(8)$ & $\mathrm{C} 29$ & $\mathrm{C} 35^{6}$ & $3.472(8)$ \\
\hline C30 & $\mathrm{C} 36^{7}$ & $3.593(7)$ & C31 & $\mathrm{C} 37^{6}$ & $3.571(7)$ \\
\hline C33 & $N 3^{6}$ & $3.572(7)$ & C33 & $\mathrm{C} 39^{6}$ & $3.517(7)$ \\
\hline C34 & $\mathrm{C} 24^{6}$ & $3.460(8)$ & C34 & $C 29^{6}$ & $3.590(8)$ \\
\hline
\end{tabular}




\begin{tabular}{|c|c|c|c|c|c|}
\hline C35 & $N 3^{7}$ & $3.381(8)$ & C35 & $\mathrm{C} 28^{6}$ & $3.452(9)$ \\
\hline C35 & $\mathrm{C} 29^{6}$ & $3.472(8)$ & C36 & $N 3^{7}$ & $3.482(7)$ \\
\hline C36 & C30 7 & $3.593(7)$ & C36 & C $39^{7}$ & $3.345(8)$ \\
\hline C37 & $\mathrm{C} 31^{6}$ & $3.571(7)$ & C37 & C38 ${ }^{7}$ & $3.365(8)$ \\
\hline C37 & C39 ${ }^{7}$ & $3.456(7)$ & C38 & $\mathrm{C} 37^{7}$ & $3.365(8)$ \\
\hline C38 & $\mathrm{C} 38^{7}$ & $3.594(7)$ & C39 & $\mathrm{C} 33^{6}$ & $3.517(7)$ \\
\hline C39 & C $36^{7}$ & $3.345(8)$ & C39 & $\mathrm{C} 37^{7}$ & $3.456(7)$ \\
\hline
\end{tabular}

Symmetry Operators:
(1) $X+1, Y, Z$
(2) $-X+1,-Y,-Z+1$
(3) $-X,-Y+1,-Z+1$
(4) $-X+1,-Y+1,-Z+1$
(5) $-X+1,-Y+1,-Z$
(6) $-X+1,-Y,-Z$
(7) $-X+2,-Y,-Z$
(8) $X, Y-1, Z$
(9) $X, Y+1, Z$
(10) $X-1, Y, Z$ 
Table 14. Intermolecular contacts less than $3.60 \AA$ involving hydrogens

\begin{tabular}{|c|c|c|c|c|c|}
\hline atom & atom & distance & atom & atom & distance \\
\hline $\mathrm{Cl1}$ & $\mathrm{H} 4$ & 3.383 & $\mathrm{Cl} 1$ & $\mathrm{H} 5$ & 3.396 \\
\hline Cl1 & $\mathrm{H} 12^{1}$ & 3.464 & Cl1 & $\mathrm{H} 13^{1}$ & 3.232 \\
\hline $\mathrm{Cl1}$ & $\mathrm{H} 23$ & 3.253 & $\mathrm{Cl} 1$ & $\mathrm{H} 27$ & 3.443 \\
\hline $\mathrm{Cl} 1$ & $\mathrm{H} 28$ & 3.527 & $\mathrm{Cl} 1$ & $\mathrm{H} 43$ & 3.349 \\
\hline $\mathrm{Cl} 2$ & $\mathrm{H} 4^{2}$ & 2.941 & $\mathrm{Cl} 2$ & $\mathrm{H} 28^{2}$ & 3.182 \\
\hline $\mathrm{Cl} 2$ & $\mathrm{H} 36^{3}$ & 2.901 & $\mathrm{Cl} 2$ & $\mathrm{H} 42$ & 3.194 \\
\hline $\mathrm{Cl} 3$ & $\mathrm{H} 5^{2}$ & 3.327 & $\mathrm{Cl} 3$ & $\mathrm{H} 13^{1}$ & 3.463 \\
\hline $\mathrm{Cl} 3$ & $\mathrm{H} 21^{4}$ & 3.347 & $\mathrm{Cl} 3$ & $\mathrm{H} 27^{2}$ & 3.251 \\
\hline N2 & $\mathrm{H} 3^{4}$ & 2.126 & N2 & $\mathrm{H} 25^{4}$ & 3.422 \\
\hline N2 & $\mathrm{H} 34^{5}$ & 3.428 & N2 & $\mathrm{H} 37^{4}$ & 2.899 \\
\hline N2 & $\mathrm{H} 46^{6}$ & 2.943 & N3 & $\mathrm{H} 35^{3}$ & 3.583 \\
\hline N4 & $\mathrm{H} 1^{7}$ & 2.130 & N4 & $\mathrm{H} 2^{7}$ & 3.148 \\
\hline N4 & $\mathrm{H} 11^{8}$ & 3.346 & N4 & $\mathrm{H} 14^{7}$ & 3.173 \\
\hline N4 & $\mathrm{H} 19^{9}$ & 2.916 & $\mathrm{C} 1$ & $\mathrm{H} 12^{1}$ & 3.438 \\
\hline $\mathrm{C} 2$ & $\mathrm{H} 11^{10}$ & 3.525 & $\mathrm{C} 2$ & $\mathrm{H} 12^{1}$ & 3.554 \\
\hline C2 & $\mathrm{H} 45^{11}$ & 3.453 & C3 & $\mathrm{H} 43$ & 3.524 \\
\hline C3 & $\mathrm{H} 43^{7}$ & 3.599 & C3 & $\mathrm{H} 45^{11}$ & 3.006 \\
\hline C4 & $\mathrm{H} 12^{10}$ & 3.378 & C4 & $\mathrm{H} 43$ & 3.599 \\
\hline C5 & $\mathrm{H} 12^{10}$ & 3.542 & C6 & $\mathrm{H} 13^{1}$ & 3.521 \\
\hline $\mathrm{C} 7$ & $\mathrm{H} 13^{1}$ & 3.417 & C9 & $\mathrm{H} 1^{10}$ & 3.520 \\
\hline C10 & $\mathrm{H} 14^{1}$ & 3.560 & C11 & $\mathrm{H} 1^{1}$ & 3.405 \\
\hline C11 & $\mathrm{H} 45^{6}$ & 3.292 & C11 & $\mathrm{H} 46^{6}$ & 3.585 \\
\hline C12 & $\mathrm{H} 1^{1}$ & 3.587 & C12 & $\mathrm{H} 45^{6}$ & 3.441 \\
\hline $\mathrm{C} 13$ & $\mathrm{H} 5^{10}$ & 3.441 & $\mathrm{C} 13$ & $\mathrm{H} 23^{1}$ & 3.466 \\
\hline C14 & $\mathrm{H} 19^{10}$ & 3.288 & C15 & $\mathrm{H} 14^{1}$ & 3.581 \\
\hline C17 & $\mathrm{H} 3^{4}$ & 3.098 & C17 & $\mathrm{H} 25^{4}$ & 3.548 \\
\hline C17 & $\mathrm{H} 46^{6}$ & 3.535 & C18 & $\mathrm{H} 25^{4}$ & 3.252 \\
\hline C19 & $\mathrm{H} 14^{10}$ & 3.233 & C19 & $\mathrm{H} 25^{4}$ & 3.550 \\
\hline
\end{tabular}




$\begin{array}{llllll}\mathrm{C} 19 & \mathrm{H} 34^{5} & 3.049 & \mathrm{C} 20 & \mathrm{H} 25^{4} & 3.547 \\ \mathrm{C} 20 & \mathrm{H} 34^{5} & 3.015 & \mathrm{C} 20 & \mathrm{H} 35^{5} & 3.223 \\ \mathrm{C} 21 & \mathrm{H} 25^{4} & 3.267 & \mathrm{C} 21 & \mathrm{H} 26 & 3.590 \\ \mathrm{C} 21 & \mathrm{H} 27 & 3.456 & \mathrm{C} 22 & \mathrm{H} 25^{4} & 2.955 \\ \mathrm{C} 22 & \mathrm{H} 26 & 3.341 & \mathrm{C} 22 & \mathrm{H} 26^{4} & 3.078 \\ \mathrm{C} 22 & \mathrm{H} 27 & 2.989 & \mathrm{C} 23 & \mathrm{H} 13^{1} & 3.574 \\ \mathrm{C} 23 & \mathrm{H} 25^{4} & 2.944 & \mathrm{C} 24 & \mathrm{H} 34^{12} & 3.452 \\ \mathrm{C} 24 & \mathrm{H} 35^{3} & 3.477 & \mathrm{C} 25 & \mathrm{H} 20^{13} & 3.333 \\ \mathrm{C} 25 & \mathrm{H} 34^{12} & 3.521 & \mathrm{C} 26 & \mathrm{H} 20^{13} & 2.983\end{array}$


Table 14. Intermolecular contacts less than $3.60 \AA$ involving hydrogens (continued)

\begin{tabular}{|c|c|c|c|c|c|}
\hline atom & atom & distance & atom & atom & distance \\
\hline C26 & $\mathrm{H} 22$ & 3.106 & C26 & $\mathrm{H} 22^{4}$ & 3.447 \\
\hline C26 & $\mathrm{H} 47$ & 3.239 & C27 & $\mathrm{H} 22$ & 3.079 \\
\hline $\mathrm{C} 27$ & $\mathrm{H} 35^{12}$ & 3.396 & $\mathrm{C} 27$ & $\mathrm{H} 47$ & 2.850 \\
\hline $\mathrm{C} 28$ & $\mathrm{H} 35^{12}$ & 3.361 & $\mathrm{C} 28$ & $\mathrm{H} 47$ & 2.846 \\
\hline $\mathrm{C} 29$ & $\mathrm{H} 36^{3}$ & 3.577 & $\mathrm{C} 29$ & $\mathrm{H} 47$ & 3.241 \\
\hline C30 & $\mathrm{H} 36^{3}$ & 3.340 & C31 & $\mathrm{H} 37^{12}$ & 3.584 \\
\hline C33 & $\mathrm{H} 37^{3}$ & 3.453 & C34 & $\mathrm{H}^{3}{ }^{3}$ & 3.438 \\
\hline C34 & $\mathrm{H} 19^{9}$ & 3.587 & C34 & $\mathrm{H} 2 \mathrm{O}^{9}$ & 3.305 \\
\hline C35 & $\mathrm{H}^{3}$ & 3.429 & C35 & $\mathrm{H} 2 \mathrm{O}^{9}$ & 3.412 \\
\hline C36 & $\mathrm{H} 46^{12}$ & 3.555 & C37 & $\mathrm{H} 46^{12}$ & 3.094 \\
\hline C38 & $\mathrm{H} 37^{3}$ & 3.404 & C39 & $\mathrm{H} 36^{3}$ & 3.473 \\
\hline $\mathrm{C} 40$ & $\mathrm{H} 1^{7}$ & 3.106 & $\mathrm{C} 40$ & $\mathrm{H} 2^{7}$ & 3.208 \\
\hline $\mathrm{C} 40$ & $\mathrm{H} 3^{12}$ & 3.586 & $\mathrm{C} 40$ & $\mathrm{H} 19^{9}$ & 3.535 \\
\hline $\mathrm{C} 41$ & $\mathrm{H} 2^{7}$ & 3.129 & C42 & $\mathrm{H} 2^{7}$ & 3.038 \\
\hline C42 & $\mathrm{H} 3 \mathrm{~A}^{7}$ & 3.424 & $\mathrm{C} 43$ & $\mathrm{H} 2^{7}$ & 3.315 \\
\hline C43 & $\mathrm{H} 3 \mathrm{~A}^{7}$ & 3.050 & C43 & $\mathrm{H} 4$ & 3.487 \\
\hline C44 & $\mathrm{H} 3 \mathrm{~A}$ & 3.543 & C44 & $\mathrm{H} 4$ & 3.535 \\
\hline C44 & $\mathrm{H} 12^{8}$ & 3.564 & C45 & $\mathrm{H} 3 \mathrm{~A}^{11}$ & 3.587 \\
\hline C45 & $\mathrm{H} 11^{8}$ & 2.955 & $\mathrm{C} 45$ & $\mathrm{H} 12^{8}$ & 3.194 \\
\hline C46 & $\mathrm{H} 2^{7}$ & 3.460 & C46 & $\mathrm{H} 11^{8}$ & 2.997 \\
\hline C46 & $\mathrm{H} 36^{12}$ & 3.555 & C46 & $\mathrm{H} 37^{12}$ & 3.184 \\
\hline $\mathrm{C} 47$ & $\mathrm{H} 42$ & 3.567 & $\mathrm{H} 1$ & $\mathrm{~N} 4^{7}$ & 2.130 \\
\hline $\mathrm{H} 1$ & $C 9^{10}$ & 3.520 & $\mathrm{H} 1$ & $\mathrm{C} 11^{1}$ & 3.405 \\
\hline $\mathrm{H} 1$ & $\mathrm{C} 12^{1}$ & 3.587 & $\mathrm{H} 1$ & $\mathrm{C} 40^{7}$ & 3.106 \\
\hline $\mathrm{H} 1$ & $\mathrm{H} 19^{10}$ & 3.274 & $\mathrm{H} 2$ & $\mathrm{~N} 4^{7}$ & 3.148 \\
\hline $\mathrm{H} 2$ & $\mathrm{C} 40^{7}$ & 3.208 & $\mathrm{H} 2$ & $\mathrm{C} 41^{7}$ & 3.129 \\
\hline $\mathrm{H} 2$ & $\mathrm{C} 42^{7}$ & 3.038 & $\mathrm{H} 2$ & $\mathrm{C} 43^{7}$ & 3.315 \\
\hline $\mathrm{H} 2$ & $\mathrm{C} 46^{7}$ & 3.460 & $\mathrm{H} 2$ & $\mathrm{H} 42^{7}$ & 3.342 \\
\hline
\end{tabular}




$\begin{array}{llllll}\mathrm{H} 2 & \mathrm{H} 45^{11} & 3.509 & \mathrm{H} 3 & \mathrm{~N} 2^{4} & 2.126 \\ \mathrm{H} 3 & \mathrm{C} 17^{4} & 3.098 & \mathrm{H} 3 & \mathrm{C} 34^{3} & 3.438 \\ \mathrm{H} 3 & {\mathrm{C} 35^{3}}^{3} & 3.429 & \mathrm{H} 3 & \mathrm{C} 40^{12} & 3.586 \\ \mathrm{H} 3 & \mathrm{H} 46^{12} & 3.529 & \mathrm{H} 3 \mathrm{~A} & \mathrm{C} 42^{7} & 3.424 \\ \mathrm{H} 3 \mathrm{~A} & \mathrm{C} 43^{7} & 3.050 & \mathrm{H} 3 \mathrm{~A} & \mathrm{C} 44 & 3.543 \\ \mathrm{H} 3 \mathrm{~A} & \mathrm{C} 45^{11} & 3.587 & \mathrm{H} 3 \mathrm{~A} & \mathrm{H} 42^{7} & 3.491 \\ \mathrm{H} 3 \mathrm{~A} & \mathrm{H} 43 & 3.124 & \mathrm{H} 3 \mathrm{~A} & \mathrm{H} 43^{7} & 2.863 \\ \mathrm{H} 3 \mathrm{~A} & \mathrm{H} 44 & 2.908 & \mathrm{H} 3 \mathrm{~A} & \mathrm{H} 45^{11} & 2.723 \\ \mathrm{H} 4 & \mathrm{Cl} 1 & 3.383 & \mathrm{H} 4 & \mathrm{Cl} 2^{14} & 2.941\end{array}$


Table 14. Intermolecular contacts less than $3.60 \AA$ involving hydrogens (continued)

\begin{tabular}{|c|c|c|c|c|c|}
\hline atom & atom & distance & atom & atom & distance \\
\hline $\mathrm{H} 4$ & C43 & 3.487 & $\mathrm{H} 4$ & C44 & 3.535 \\
\hline $\mathrm{H} 4$ & $\mathrm{H} 12^{10}$ & 3.538 & $\mathrm{H} 4$ & $\mathrm{H} 43$ & 3.265 \\
\hline $\mathrm{H} 4$ & $\mathrm{H} 44$ & 3.353 & H5 & $\mathrm{Cl} 1$ & 3.396 \\
\hline H5 & $\mathrm{Cl}^{14}$ & 3.327 & H5 & $\mathrm{C} 13^{10}$ & 3.441 \\
\hline H5 & $\mathrm{H} 13^{10}$ & 3.379 & H5 & $\mathrm{H} 27$ & 3.535 \\
\hline H11 & $N 4^{6}$ & 3.346 & $\mathrm{H} 11$ & $C 2^{10}$ & 3.525 \\
\hline $\mathrm{H} 11$ & $C 45^{6}$ & 2.955 & $\mathrm{H} 11$ & $C 46^{6}$ & 2.997 \\
\hline $\mathrm{H} 11$ & $\mathrm{H} 45^{6}$ & 2.621 & H11 & $\mathrm{H} 46^{6}$ & 2.680 \\
\hline $\mathrm{H} 12$ & $\mathrm{Cl}^{1}$ & 3.464 & $\mathrm{H} 12$ & $\mathrm{C} 1^{1}$ & 3.438 \\
\hline $\mathrm{H} 12$ & $C 2^{1}$ & 3.554 & $\mathrm{H} 12$ & $\mathrm{C} 4^{10}$ & 3.378 \\
\hline $\mathrm{H} 12$ & $C 5^{10}$ & 3.542 & $\mathrm{H} 12$ & $C 44^{6}$ & 3.564 \\
\hline $\mathrm{H} 12$ & $\mathrm{C} 45^{6}$ & 3.194 & $\mathrm{H} 12$ & $\mathrm{H} 4^{10}$ & 3.538 \\
\hline $\mathrm{H} 12$ & $\mathrm{H} 44^{6}$ & 3.553 & $\mathrm{H} 12$ & $\mathrm{H} 45^{6}$ & 2.925 \\
\hline $\mathrm{H} 13$ & $\mathrm{Cl}^{1}$ & 3.232 & $\mathrm{H} 13$ & $\mathrm{Cl}^{1}$ & 3.463 \\
\hline $\mathrm{H} 13$ & $\mathrm{C} 6^{1}$ & 3.521 & $\mathrm{H} 13$ & $C 7^{1}$ & 3.417 \\
\hline $\mathrm{H} 13$ & $\mathrm{C} 23^{1}$ & 3.574 & $\mathrm{H} 13$ & $\mathrm{H} 5^{10}$ & 3.379 \\
\hline $\mathrm{H} 13$ & $\mathrm{H} 23^{1}$ & 2.672 & $\mathrm{H} 14$ & $\mathrm{~N} 4^{7}$ & 3.173 \\
\hline H14 & $\mathrm{C} 10^{1}$ & 3.560 & $\mathrm{H} 14$ & $\mathrm{C} 15^{1}$ & 3.581 \\
\hline $\mathrm{H} 14$ & $\mathrm{C} 19^{10}$ & 3.233 & $\mathrm{H} 14$ & $\mathrm{H} 19^{10}$ & 2.596 \\
\hline H19 & $N 4^{5}$ & 2.916 & $\mathrm{H} 19$ & $\mathrm{C} 14^{10}$ & 3.288 \\
\hline $\mathrm{H} 19$ & $\mathrm{C} 34^{5}$ & 3.587 & $\mathrm{H} 19$ & $C 40^{5}$ & 3.535 \\
\hline H19 & $\mathrm{H} 1^{10}$ & 3.274 & $\mathrm{H} 19$ & $\mathrm{H} 14^{10}$ & 2.596 \\
\hline $\mathrm{H} 19$ & $\mathrm{H} 34^{5}$ & 2.702 & $\mathrm{H} 2 \mathrm{O}$ & $\mathrm{C} 25^{13}$ & 3.333 \\
\hline $\mathrm{H} 2 \mathrm{O}$ & $\mathrm{C} 26^{13}$ & 2.983 & $\mathrm{H} 2 \mathrm{O}$ & $\mathrm{C} 34^{5}$ & 3.305 \\
\hline $\mathrm{H} 2 \mathrm{O}$ & $\mathrm{C} 35^{5}$ & 3.412 & $\mathrm{H} 2 \mathrm{O}$ & $\mathrm{H} 25^{13}$ & 3.450 \\
\hline $\mathrm{H} 2 \mathrm{O}$ & $\mathrm{H} 26^{13}$ & 2.851 & $\mathrm{H} 2 \mathrm{O}$ & $\mathrm{H} 34^{5}$ & 2.628 \\
\hline $\mathrm{H} 2 \mathrm{O}$ & $\mathrm{H} 35^{5}$ & 2.859 & $\mathrm{H} 21$ & $\mathrm{Cl}^{4}$ & 3.347 \\
\hline $\mathrm{H} 21$ & $\mathrm{H} 21^{13}$ & 2.931 & $\mathrm{H} 21$ & $\mathrm{H} 26$ & 3.114 \\
\hline
\end{tabular}




$\begin{array}{llllll}\mathrm{H} 21 & \mathrm{H} 27 & 3.407 & \mathrm{H} 22 & \mathrm{C} 26 & 3.106 \\ \mathrm{H} 22 & \mathrm{C} 26^{4} & 3.447 & \mathrm{H} 22 & \mathrm{C} 27 & 3.079 \\ \mathrm{H} 22 & \mathrm{H} 25^{4} & 3.310 & \mathrm{H} 22 & \mathrm{H} 26 & 2.617 \\ \mathrm{H} 22 & \mathrm{H} 26^{4} & 2.737 & \mathrm{H} 22 & \mathrm{H} 27 & 2.574 \\ \mathrm{H} 23 & \mathrm{Cl} 1 & 3.253 & \mathrm{H} 23 & \mathrm{C} 13^{1} & 3.466 \\ \mathrm{H} 23 & \mathrm{H} 13^{1} & 2.672 & \mathrm{H} 23 & \mathrm{H} 25^{4} & 3.296 \\ \mathrm{H} 25 & \mathrm{~N} 2^{4} & 3.422 & \mathrm{H} 25 & \mathrm{C} 17^{4} & 3.548 \\ \mathrm{H} 25 & \mathrm{C} 18^{4} & 3.252 & \mathrm{H} 25 & \mathrm{C} 19^{4} & 3.550 \\ \mathrm{H} 25 & \mathrm{C} 20^{4} & 3.547 & \mathrm{H} 25 & \mathrm{C} 21^{4} & 3.267\end{array}$


Table 14. Intermolecular contacts less than $3.60 \AA$ involving hydrogens (continued)

\begin{tabular}{|c|c|c|c|c|c|}
\hline atom & atom & distance & atom & atom & distance \\
\hline $\mathrm{H} 25$ & $\mathrm{C} 22^{4}$ & 2.955 & $\mathrm{H} 25$ & $\mathrm{C} 23^{4}$ & 2.944 \\
\hline $\mathrm{H} 25$ & $\mathrm{H} 2 \mathrm{O}^{13}$ & 3.450 & $\mathrm{H} 25$ & $\mathrm{H} 22^{4}$ & 3.310 \\
\hline $\mathrm{H} 25$ & $\mathrm{H} 23^{4}$ & 3.296 & $\mathrm{H} 26$ & $\mathrm{C} 21$ & 3.590 \\
\hline $\mathrm{H} 26$ & $\mathrm{C} 22$ & 3.341 & $\mathrm{H} 26$ & $\mathrm{C} 22^{4}$ & 3.078 \\
\hline $\mathrm{H} 26$ & $\mathrm{H} 2 \mathrm{O}^{13}$ & 2.851 & $\mathrm{H} 26$ & $\mathrm{H} 21$ & 3.114 \\
\hline H26 & $\mathrm{H} 22$ & 2.617 & $\mathrm{H} 26$ & $\mathrm{H} 22^{4}$ & 2.737 \\
\hline $\mathrm{H} 27$ & $\mathrm{Cl} 1$ & 3.443 & $\mathrm{H} 27$ & $\mathrm{Cl}^{14}$ & 3.251 \\
\hline $\mathrm{H} 27$ & C21 & 3.456 & $\mathrm{H} 27$ & $\mathrm{C} 22$ & 2.989 \\
\hline $\mathrm{H} 27$ & H5 & 3.535 & $\mathrm{H} 27$ & $\mathrm{H} 21$ & 3.407 \\
\hline $\mathrm{H} 27$ & $\mathrm{H} 22$ & 2.574 & $\mathrm{H} 27$ & $\mathrm{H} 47$ & 3.172 \\
\hline $\mathrm{H} 28$ & $\mathrm{Cl} 1$ & 3.527 & $\mathrm{H} 28$ & $\mathrm{Cl} 2^{14}$ & 3.182 \\
\hline $\mathrm{H} 28$ & $\mathrm{H} 36^{12}$ & 3.540 & $\mathrm{H} 28$ & $\mathrm{H} 47$ & 3.159 \\
\hline H34 & $\mathrm{N} 2^{9}$ & 3.428 & H34 & $\mathrm{C} 19^{9}$ & 3.049 \\
\hline H34 & $\mathrm{C} 20^{9}$ & 3.015 & H34 & $\mathrm{C} 24^{12}$ & 3.452 \\
\hline H34 & $\mathrm{C} 25^{12}$ & 3.521 & H34 & $\mathrm{H} 19^{9}$ & 2.702 \\
\hline H34 & $\mathrm{H} 2 \mathrm{O}^{9}$ & 2.628 & H35 & $\mathrm{N}^{3}$ & 3.583 \\
\hline H35 & $\mathrm{C} 20^{9}$ & 3.223 & H35 & $\mathrm{C} 24^{3}$ & 3.477 \\
\hline H35 & $\mathrm{C} 27^{12}$ & 3.396 & H35 & $\mathrm{C} 28^{12}$ & 3.361 \\
\hline H35 & $\mathrm{H} 2 \mathrm{O}^{9}$ & 2.859 & H35 & $\mathrm{H} 47^{3}$ & 3.246 \\
\hline H36 & $\mathrm{Cl} 2^{3}$ & 2.901 & H36 & $\mathrm{C} 29^{3}$ & 3.577 \\
\hline H36 & $\mathrm{C} 30^{3}$ & 3.340 & H36 & $\mathrm{C} 39^{3}$ & 3.473 \\
\hline H36 & $\mathrm{C} 46^{12}$ & 3.555 & H36 & $\mathrm{H} 28^{12}$ & 3.540 \\
\hline H36 & $\mathrm{H} 42^{3}$ & 3.114 & H36 & $\mathrm{H} 46^{12}$ & 3.369 \\
\hline H36 & $\mathrm{H} 47^{3}$ & 3.309 & H37 & $\mathrm{N} 2^{4}$ & 2.899 \\
\hline H37 & $\mathrm{C} 31^{12}$ & 3.584 & H37 & $\mathrm{C} 33^{3}$ & 3.453 \\
\hline H37 & $\mathrm{C} 38^{3}$ & 3.404 & H37 & $\mathrm{C} 46^{12}$ & 3.184 \\
\hline H37 & $\mathrm{H} 46^{12}$ & 2.485 & $\mathrm{H} 42$ & $\mathrm{Cl} 2$ & 3.194 \\
\hline $\mathrm{H} 42$ & $\mathrm{C} 47$ & 3.567 & $\mathrm{H} 42$ & $\mathrm{H} 2^{7}$ & 3.342 \\
\hline
\end{tabular}




$\begin{array}{llllll}\mathrm{H} 42 & \mathrm{H}_{3} \mathrm{~A}^{7} & 3.491 & \mathrm{H} 42 & \mathrm{H} 36^{3} & 3.114 \\ \mathrm{H} 42 & \mathrm{H} 47 & 3.300 & \mathrm{H} 43 & \mathrm{Cl} 1 & 3.349 \\ \mathrm{H} 43 & \mathrm{C} 3 & 3.524 & \mathrm{H} 43 & \mathrm{C}^{7} & 3.599 \\ \mathrm{H} 43 & \mathrm{C} 4 & 3.599 & \mathrm{H} 43 & \mathrm{H} 3 \mathrm{~A} & 3.124 \\ \mathrm{H} 43 & \mathrm{H}^{7} \mathrm{~A}^{7} & 2.863 & \mathrm{H} 43 & \mathrm{H} 4 & 3.265 \\ \mathrm{H} 43 & \mathrm{H} 43^{7} & 3.094 & \mathrm{H} 43 & \mathrm{H} 44^{7} & 3.197 \\ \mathrm{H} 44 & \mathrm{H} 3 \mathrm{~A} & 2.908 & \mathrm{H} 44 & \mathrm{H} 4 & 3.353 \\ \mathrm{H} 44 & \mathrm{H} 12^{8} & 3.553 & \mathrm{H} 44 & \mathrm{H} 43^{7} & 3.197 \\ \mathrm{H} 45 & \mathrm{C} 2^{11} & 3.453 & \mathrm{H} 45 & \mathrm{C}^{11} & 3.006\end{array}$


Table 14. Intermolecular contacts less than $3.60 \AA$ involving hydrogens (continued)

$\begin{array}{llllll}\text { atom } & \text { atom } & \text { distance } & \text { atom } & \text { atom } & \text { distance } \\ \mathrm{H} 45 & \mathrm{C} 11^{8} & 3.292 & \mathrm{H} 45 & \mathrm{C}^{8} 2^{8} & 3.441 \\ \mathrm{H} 45 & \mathrm{H} 2^{11} & 3.509 & \mathrm{H} 45 & \mathrm{H}^{11} \mathrm{~A}^{11} & 2.723 \\ \mathrm{H} 45 & \mathrm{H} 11^{8} & 2.621 & \mathrm{H} 45 & \mathrm{H} 12^{8} & 2.925 \\ \mathrm{H} 46 & \mathrm{~N} 2^{8} & 2.943 & \mathrm{H} 46 & \mathrm{C} 11^{8} & 3.585 \\ \mathrm{H} 46 & \mathrm{C} 17^{8} & 3.535 & \mathrm{H} 46 & \mathrm{C} 36^{12} & 3.555 \\ \mathrm{H} 46 & \mathrm{C} 37^{12} & 3.094 & \mathrm{H} 46 & \mathrm{H} 3^{12} & 3.529 \\ \mathrm{H} 46 & \mathrm{H} 11^{8} & 2.680 & \mathrm{H} 46 & \mathrm{H} 36^{12} & 3.369 \\ \mathrm{H} 46 & \mathrm{H} 37^{12} & 2.485 & \mathrm{H} 47 & \mathrm{C} 26 & 3.239 \\ \mathrm{H} 47 & \mathrm{C} 27 & 2.850 & \mathrm{H} 47 & \mathrm{C} 28 & 2.846 \\ \mathrm{H} 47 & \mathrm{C} 29 & 3.241 & \mathrm{H} 47 & \mathrm{H} 27 & 3.172 \\ \mathrm{H} 47 & \mathrm{H} 28 & 3.159 & \mathrm{H} 47 & \mathrm{H} 35^{3} & 3.246 \\ \mathrm{H} 47 & \mathrm{H} 36^{3} & 3.309 & \mathrm{H} 47 & \mathrm{H} 42 & 3.300\end{array}$

Symmetry Operators:
(1) $-X+1,-Y+1,-Z+1$
(2) $X+1, Y, Z$
(3) $-X+2,-Y,-Z$
(4) $-X+1,-Y+1,-Z$
(5) $X-1, Y+1, Z$
(6) $X, Y+1, Z$
(7) $-X+1,-Y,-Z+1$
(8) $X, Y-1, Z$
(9) $X+1, Y-1, Z$
(10) $-X,-Y+1,-Z+1$
(11) $-X,-Y,-Z+1$
(12) $-X+1,-Y,-Z$
(13) $-X,-Y+1,-Z$
(14) $X-1, Y, Z$ 


\section{Reference}

(1) Yasuhara, A.; Kanamori, Y.; Kaneko, M.; Numata, A.; Kondo, Y.; Sakamoto, T.; J. Chem. Soc., Perkin Trans. 1999, 1, 529-534

(2) Yamakawa, T.; Ideue, E.; Shimokawa, J.; Fukuyama, T.; Angew. Chem. Int. Ed., 2010, 49, 9262-9265

(3) Rainier, J. D.; Kennedy, A. R.; J. Org. Chem., 2000, 65, 6213-6216

(4) Kim, T.; Kim, K.; Tetrahedron Lett., 2010, 51, 868-871

(5) Xu, M.; Hou, Q.; Wang, S.; Yao, Z.; Wang, H.; SYNTHESIS, 2011, 4, 626-634 


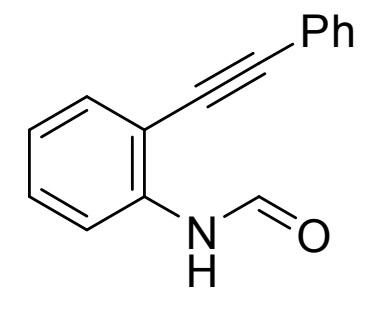

S1

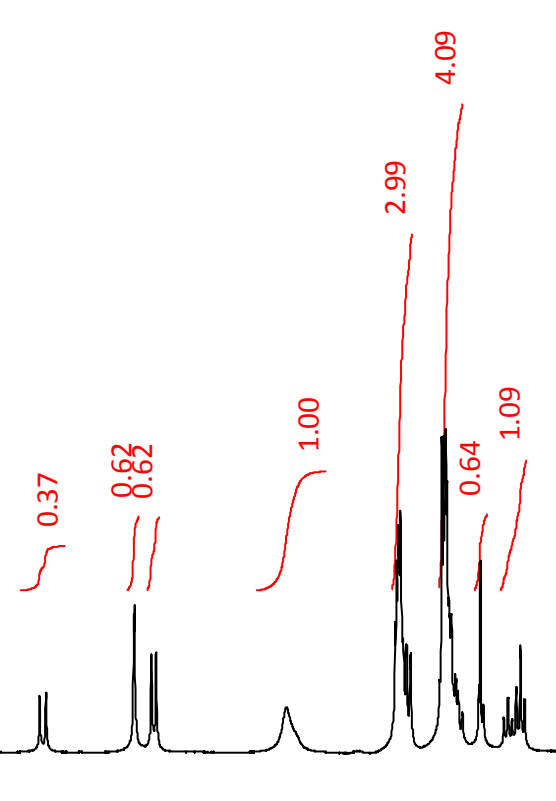




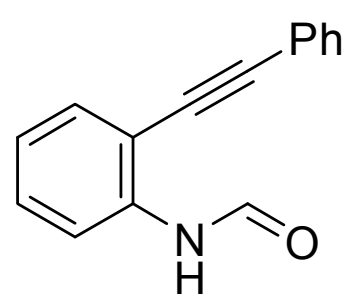

S1

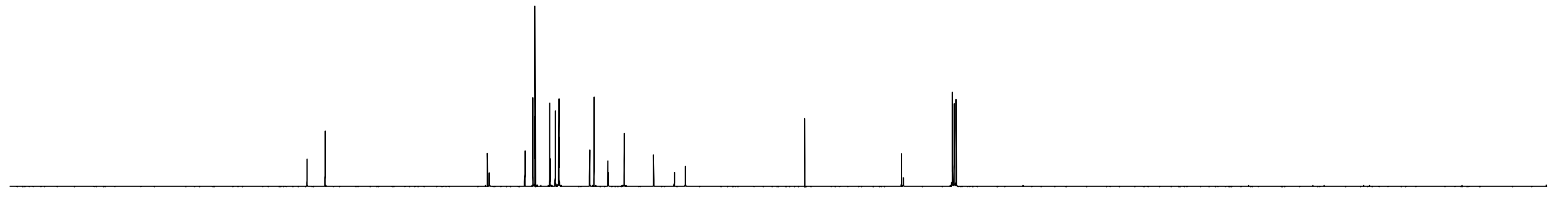

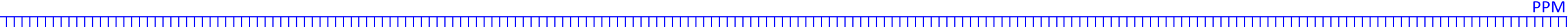
190
$180 \quad 170$

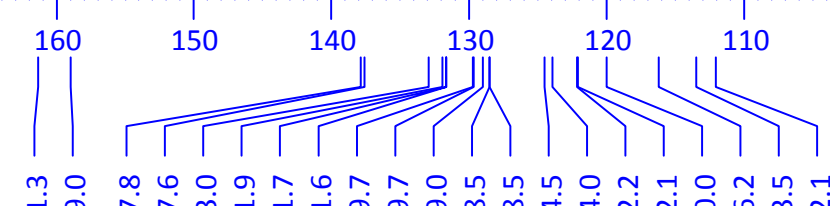

100

90

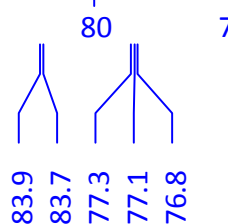

60

50

40

30

20

10

0

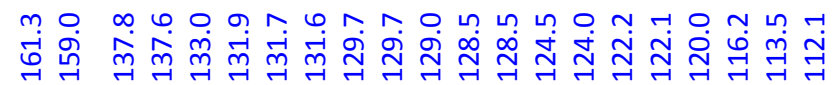



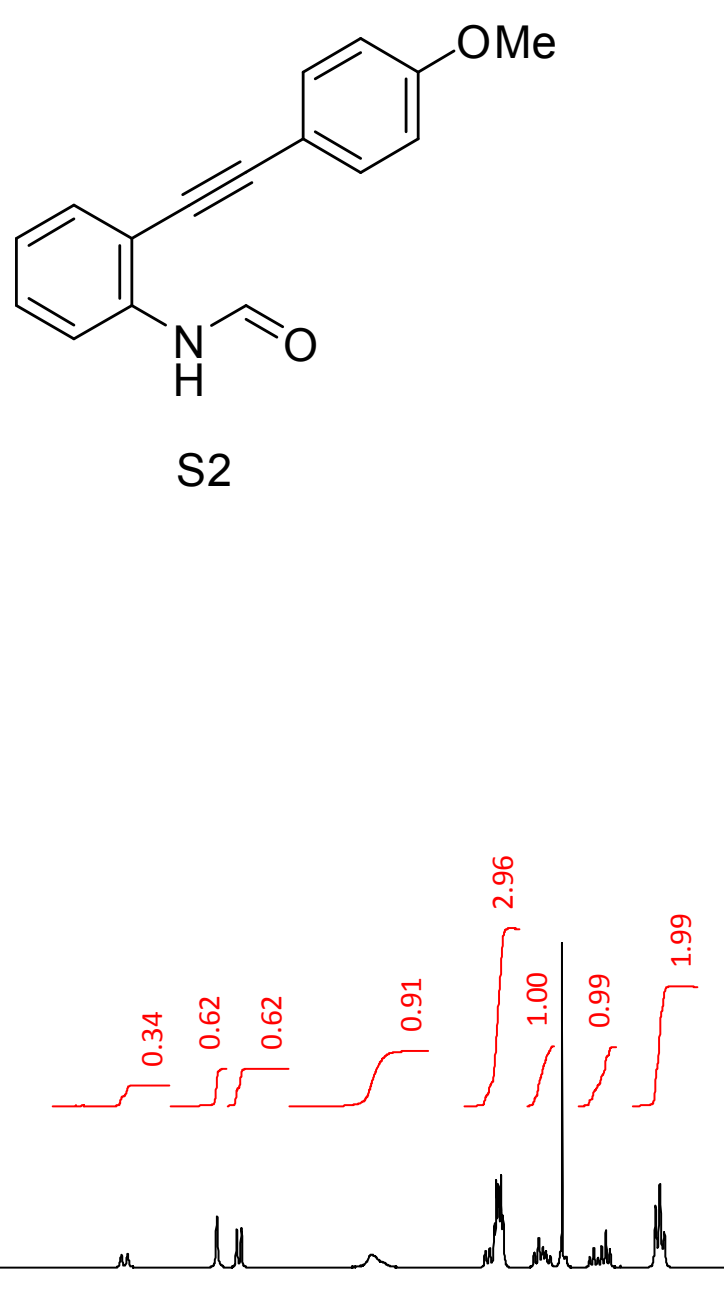

9.0

$111|1| 1|1|$

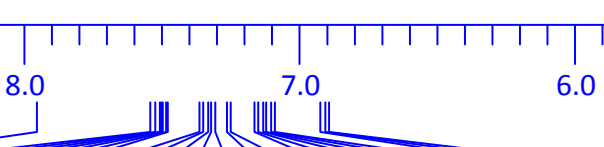

6.0

$\left.\right|_{5.0} ^{1}$

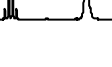

4.0

4.0 s.

r

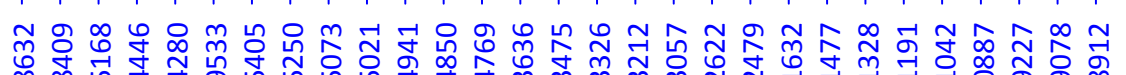

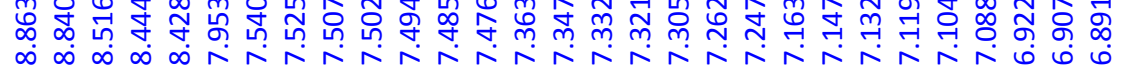




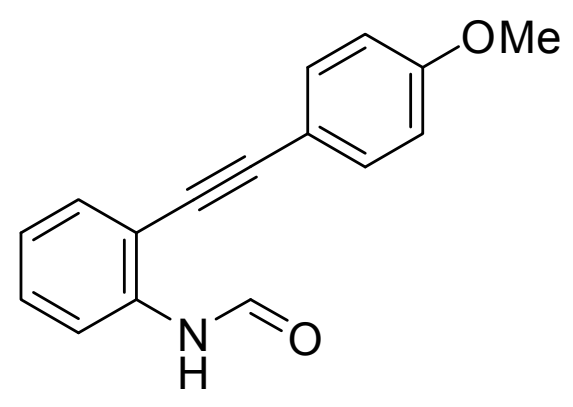

S2

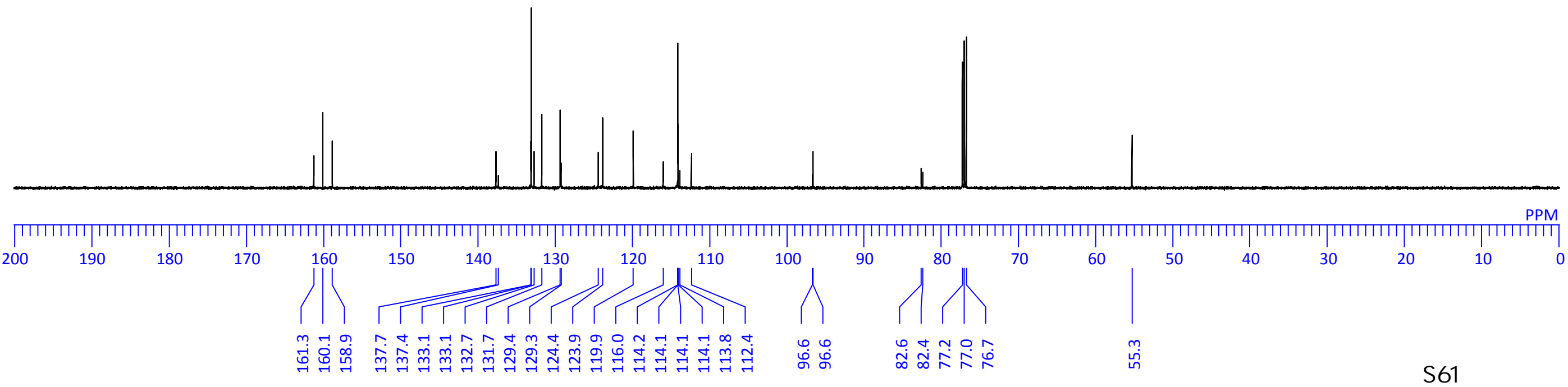




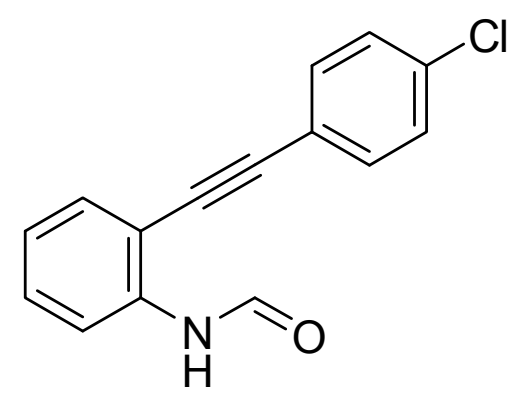

S3

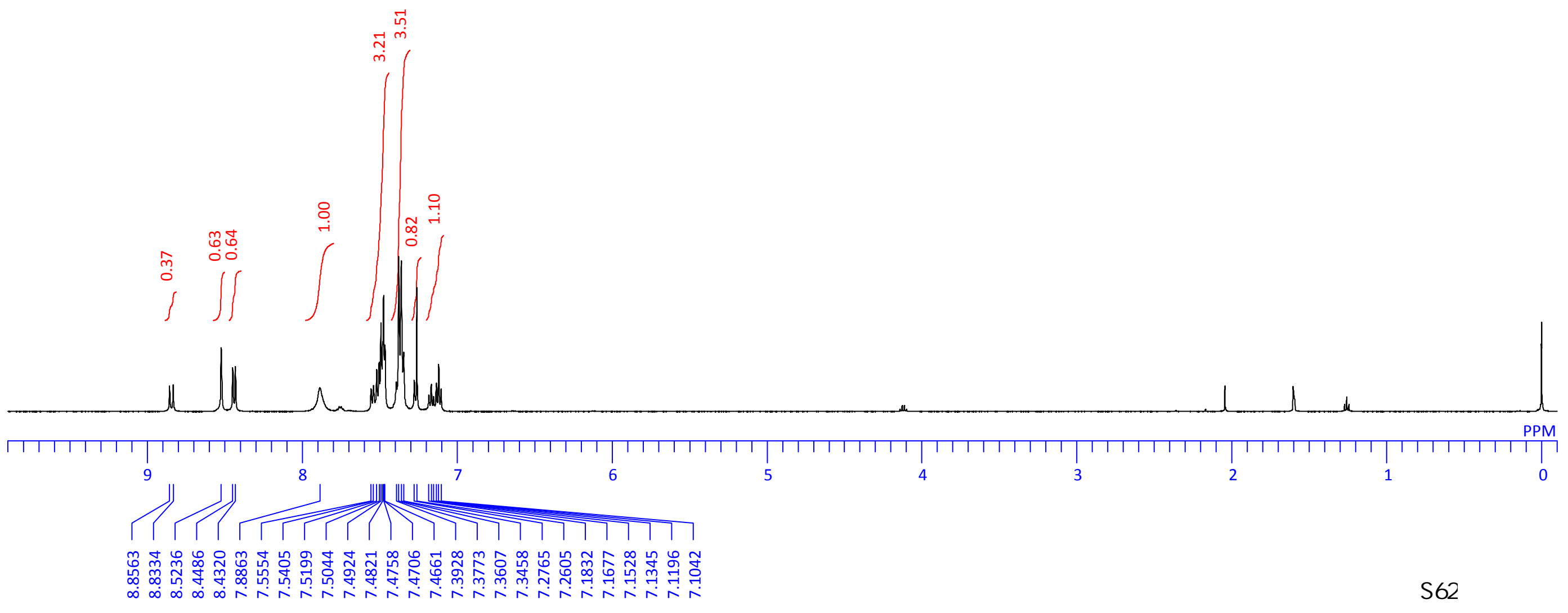




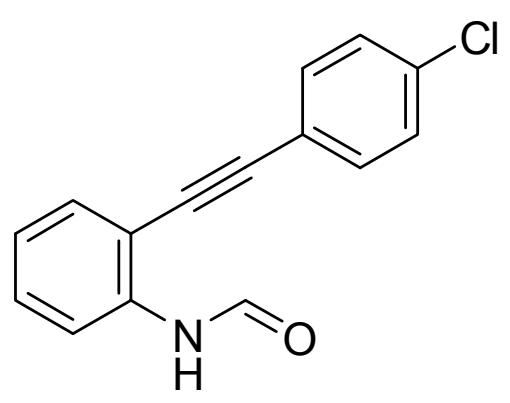

S3

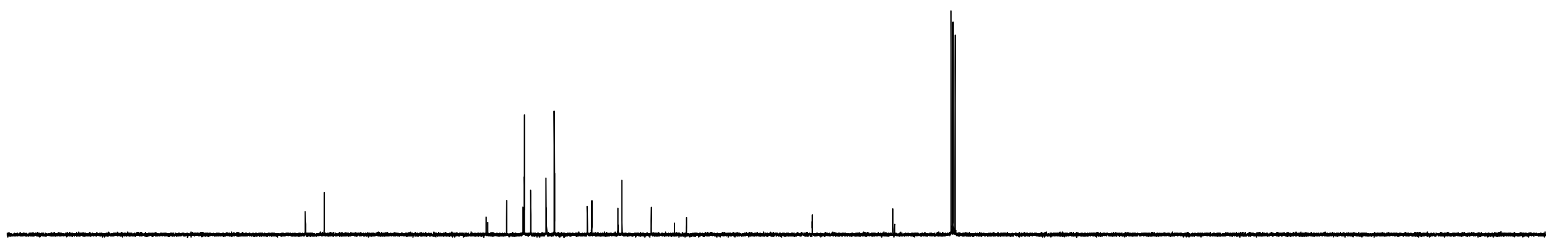
2
$190 \quad 180$
170

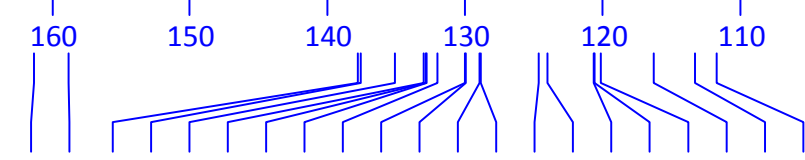

$100 \quad 90$

$90 \quad 80$

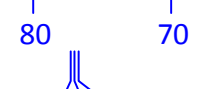

60

50

40

30

20

10

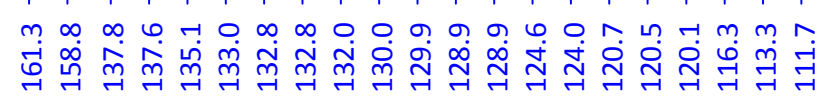

นุ่ nू.

○

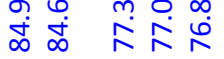




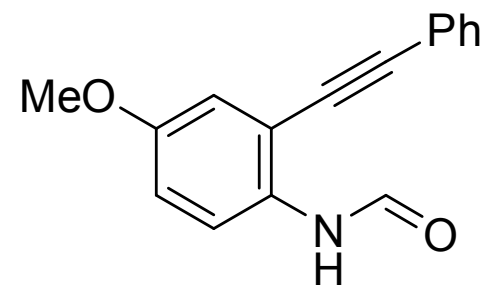

S4

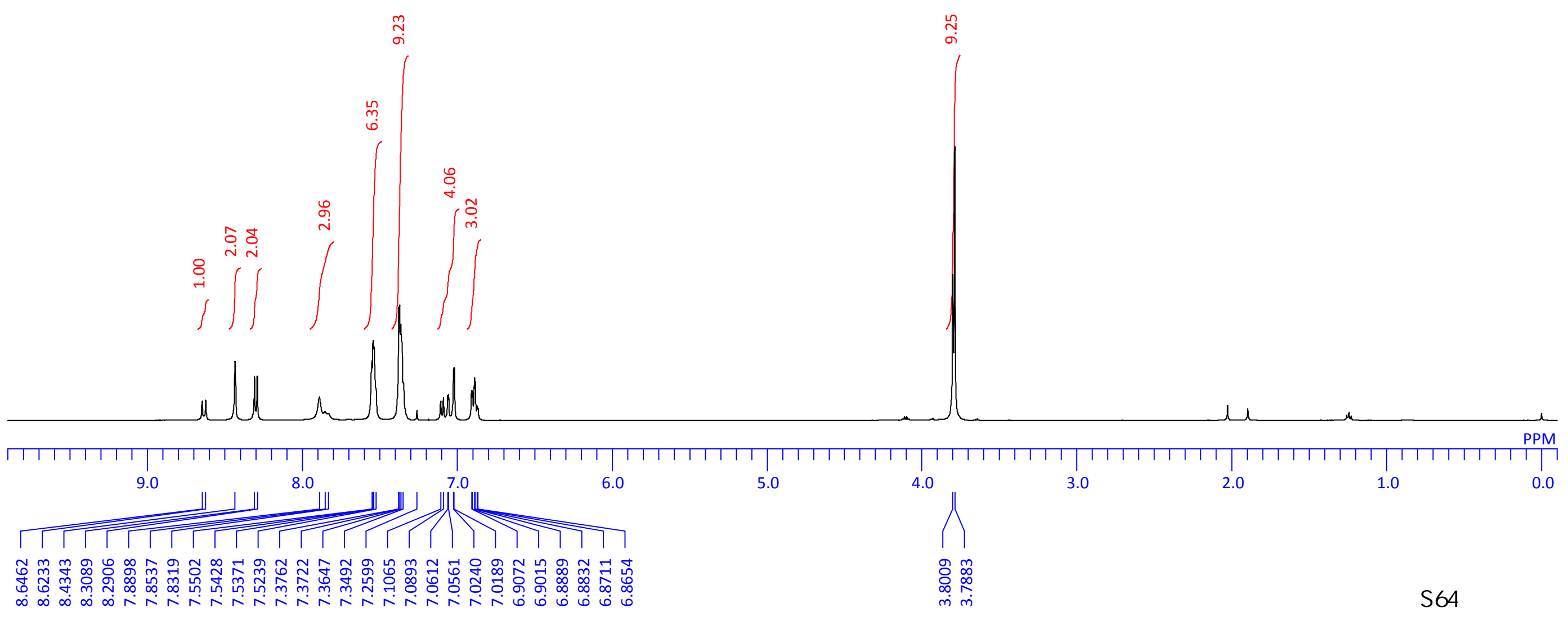




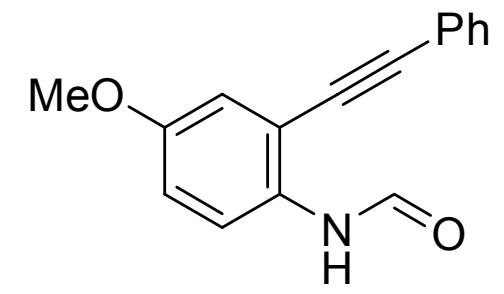

S4

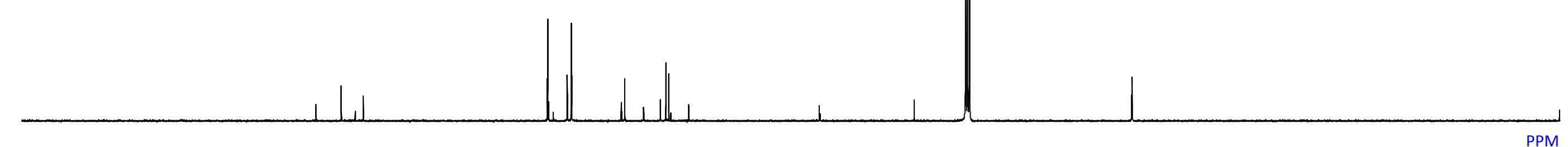

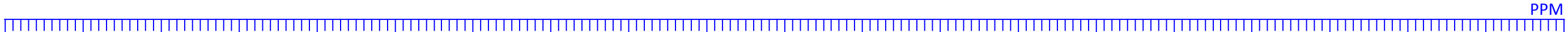

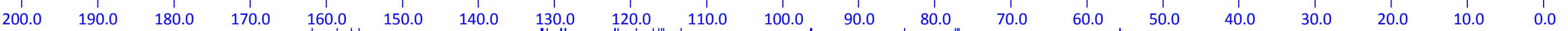

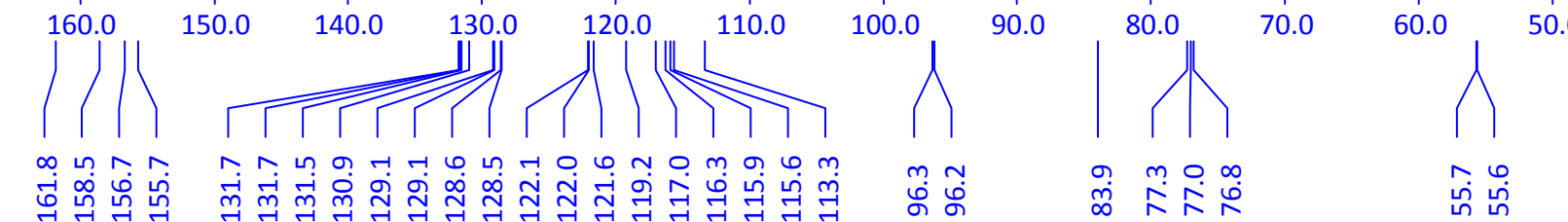




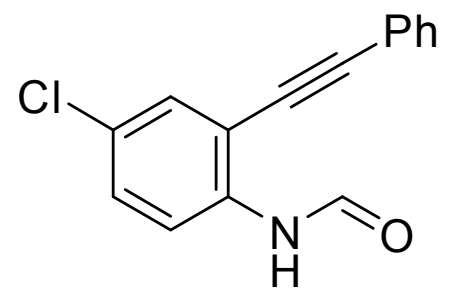

S5

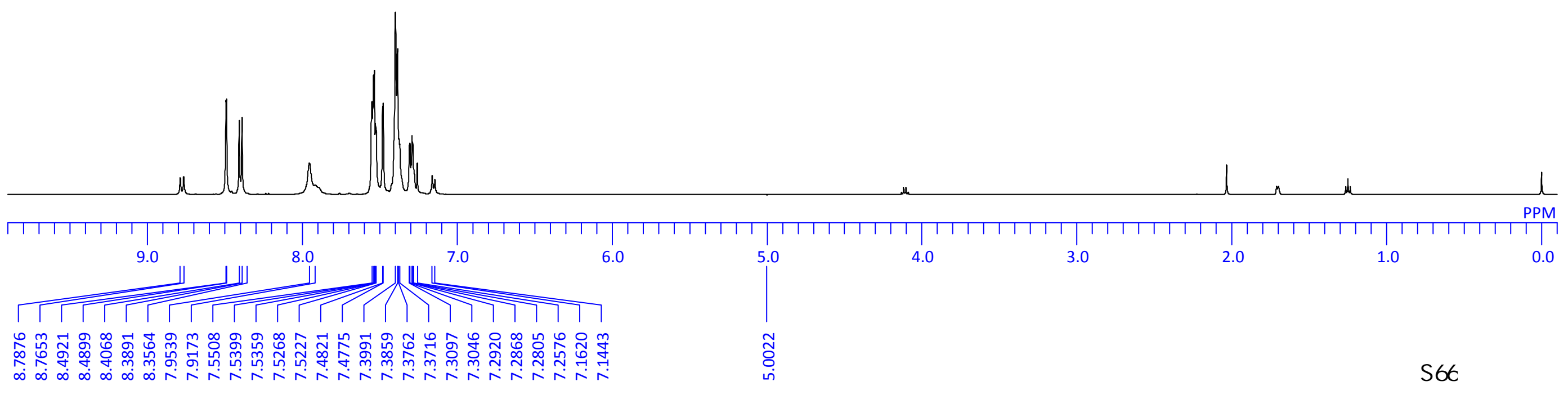




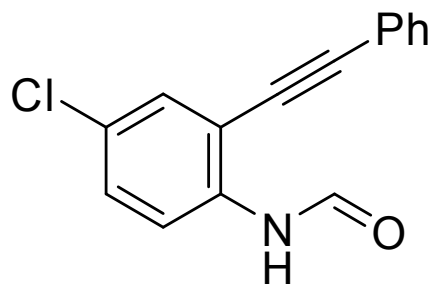

S5

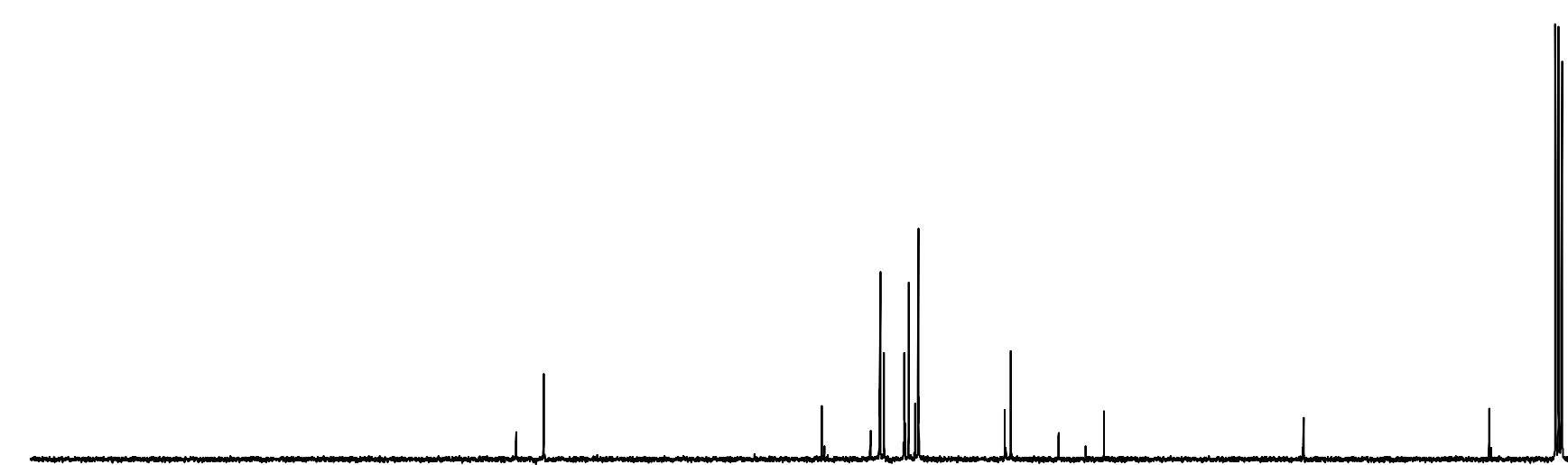

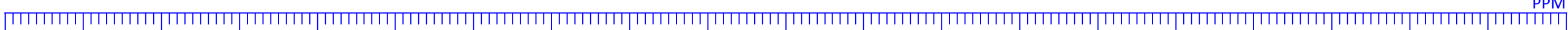

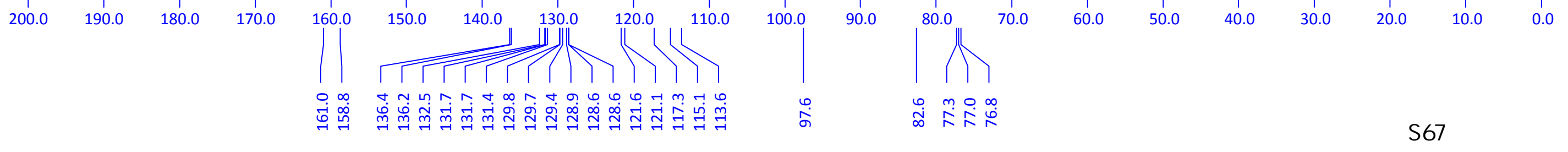




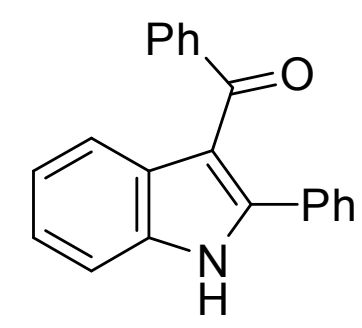

$1 a$

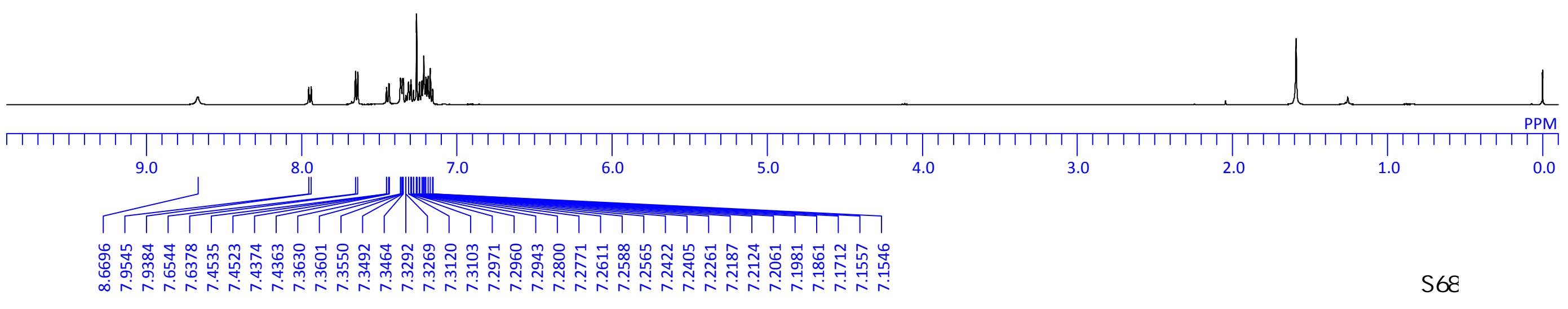




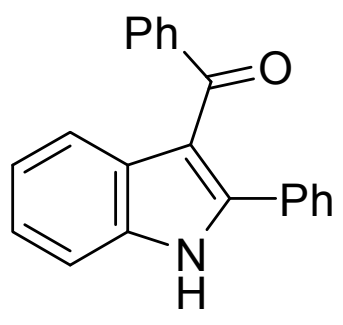

$1 a$

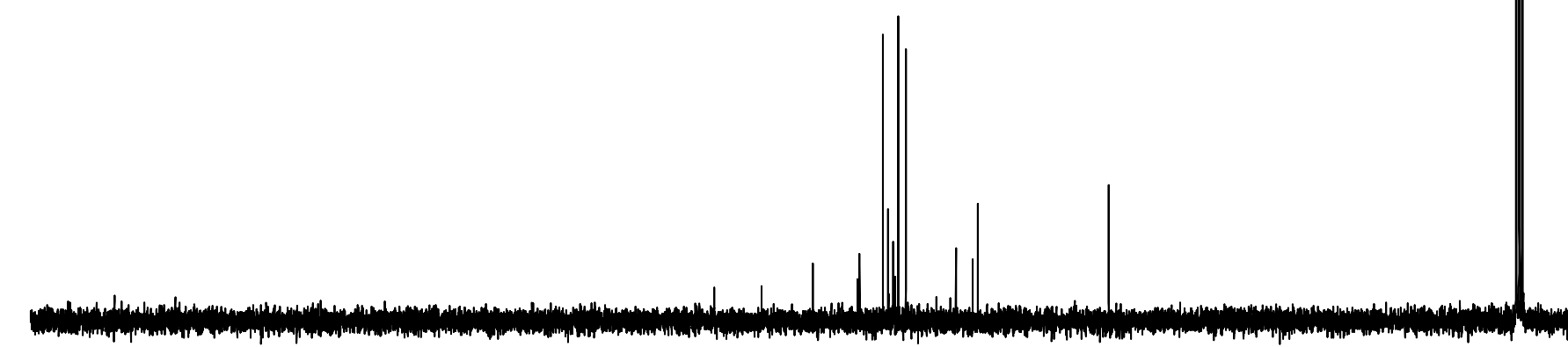

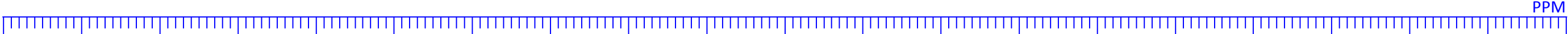
$190.0 \quad 180$
$170.0 \quad 160.0$
100.0
90.0
60.0
50.0
40.0
30.0
20.0
10.0
0.0
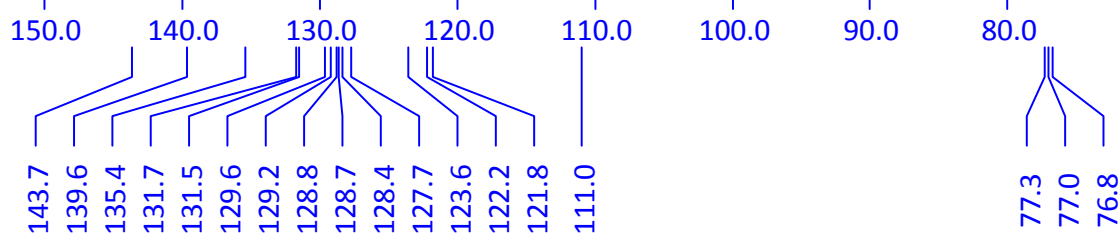

m요숭 


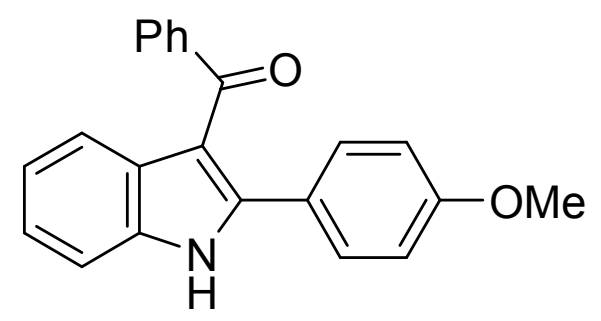

$1 b$

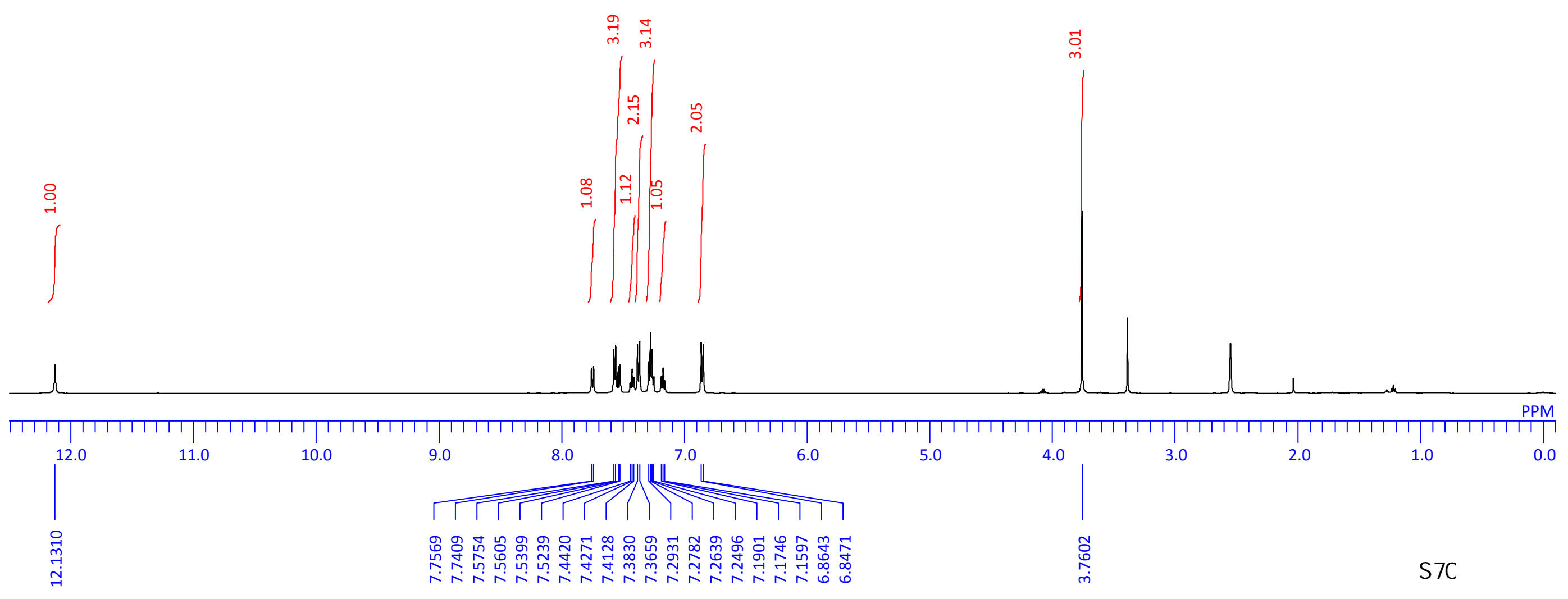




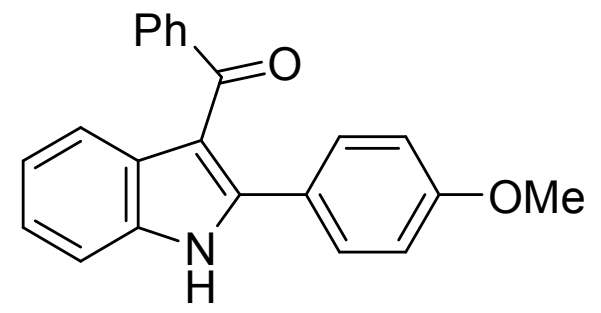

$1 b$

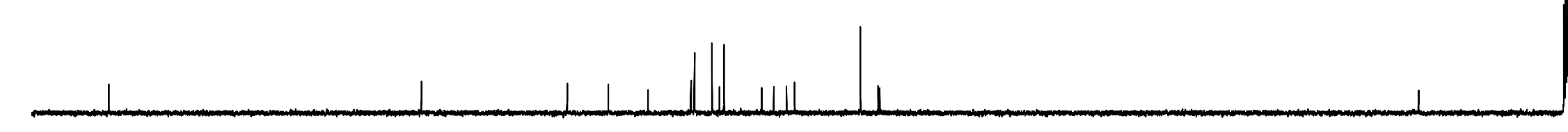

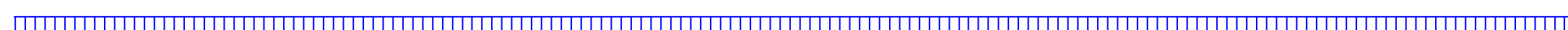
200.0

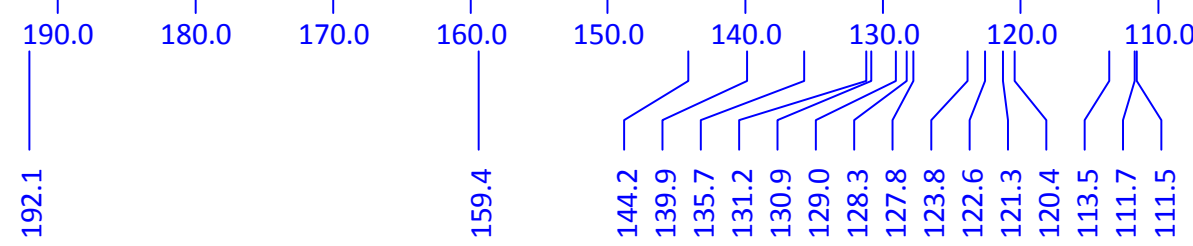

100.0

$90.0 \quad 80.0$

70.0

60.0
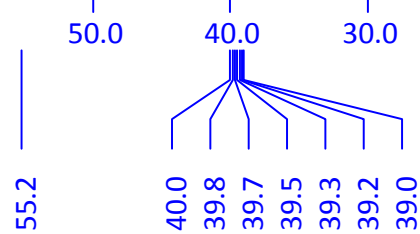

20.0

10.0 


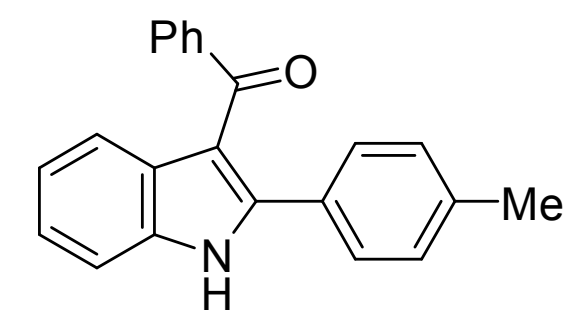

$1 c$

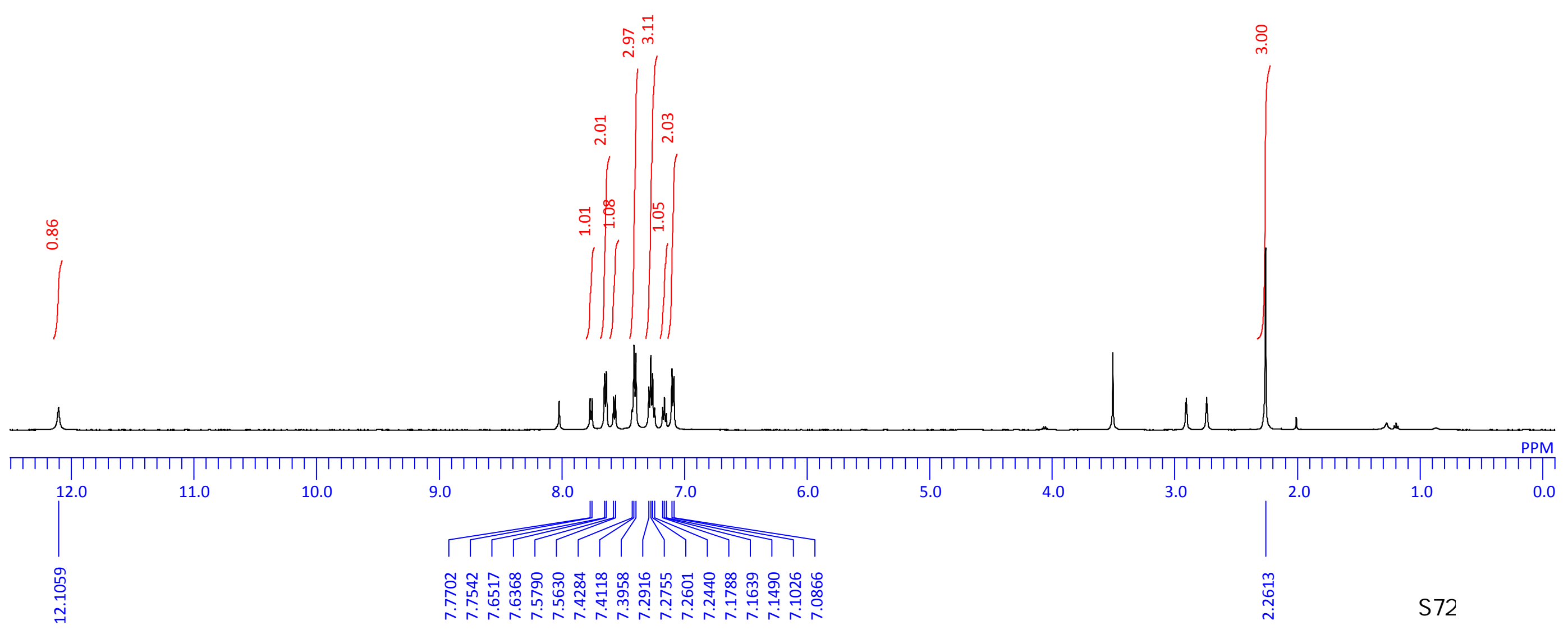




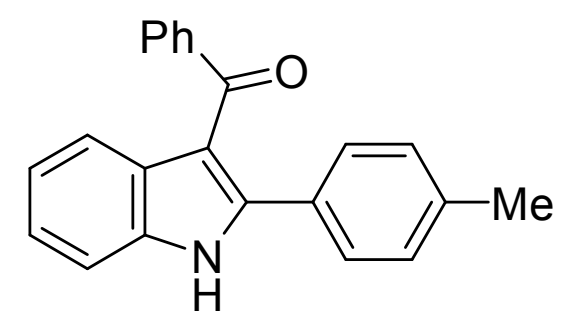

$1 c$

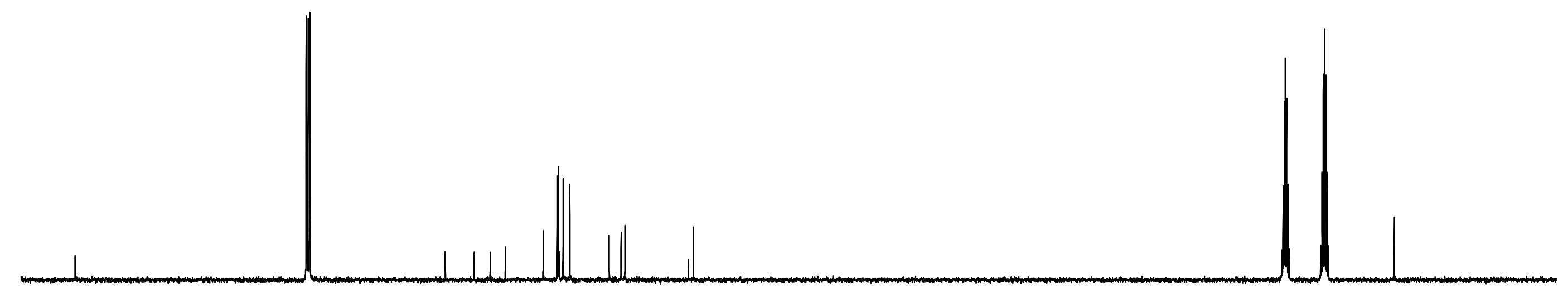

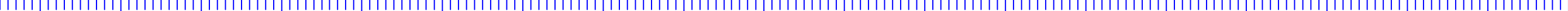
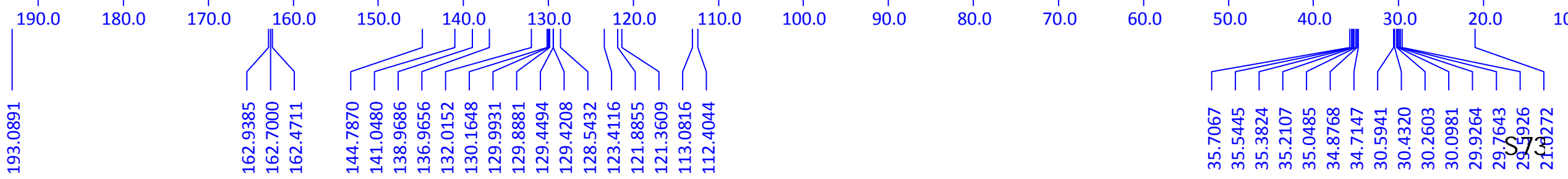


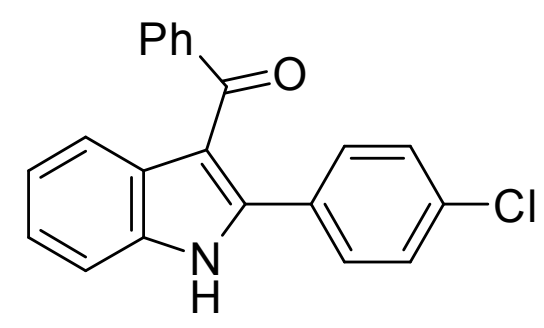

1d

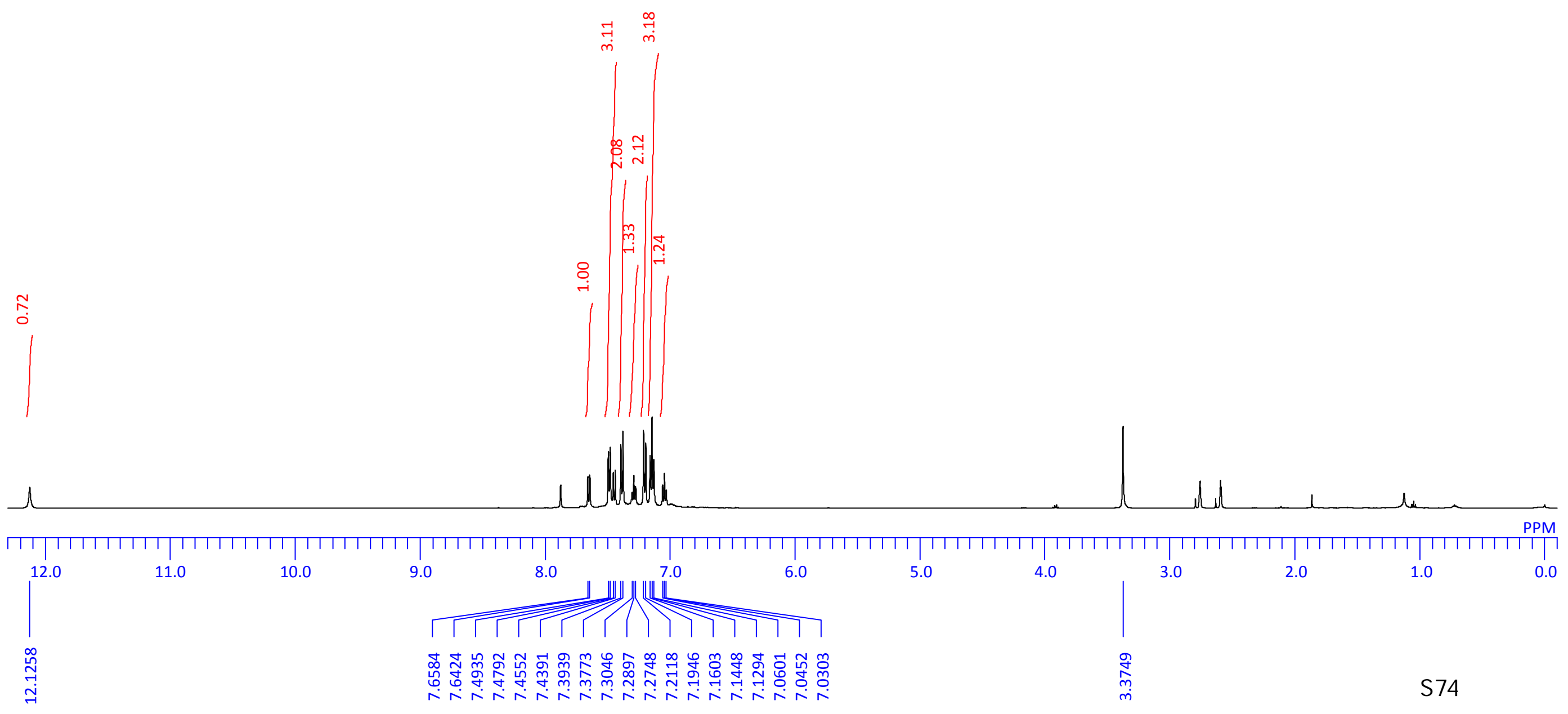




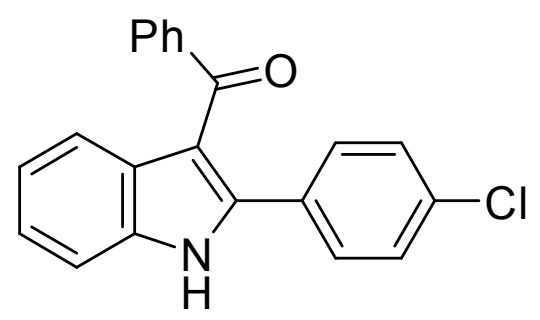

$1 d$

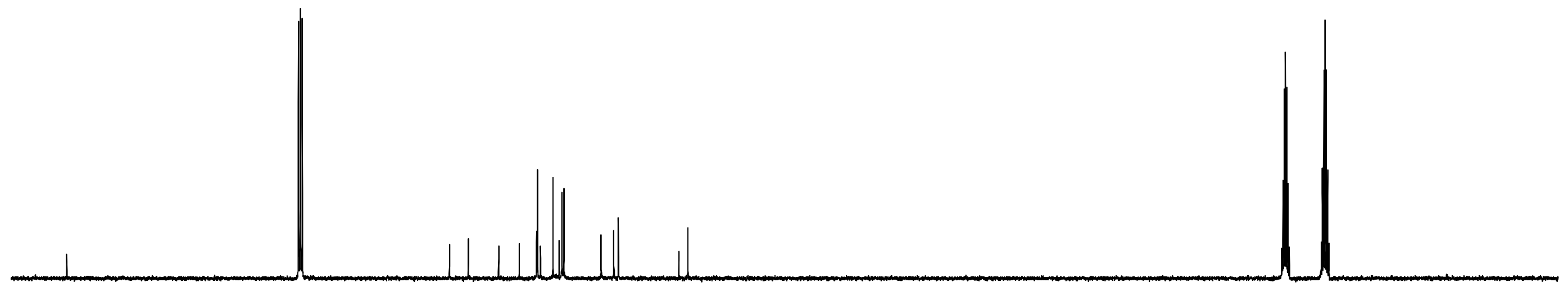

एकासापा

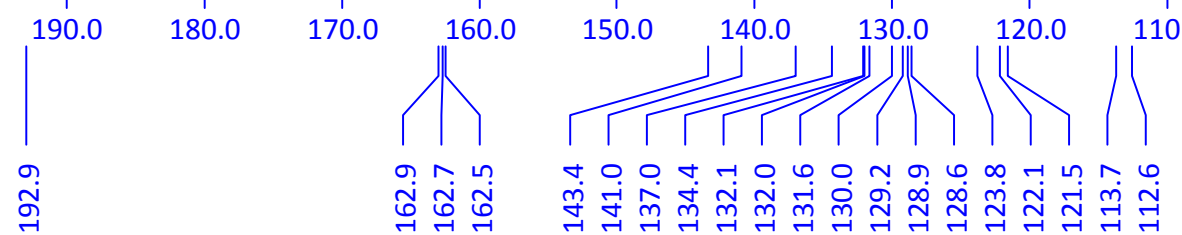

$70.0 \quad 60.0$

$\prod_{50.0}$

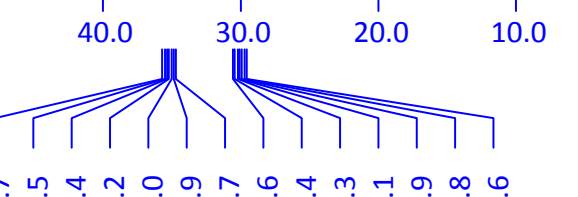

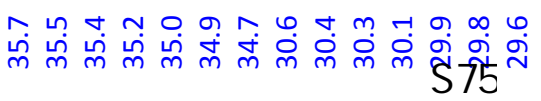


<smiles>O=C(c1ccccc1)c1c(-c2ccc(C(F)(F)F)cc2)[nH]c2ccccc12</smiles>

$1 e$

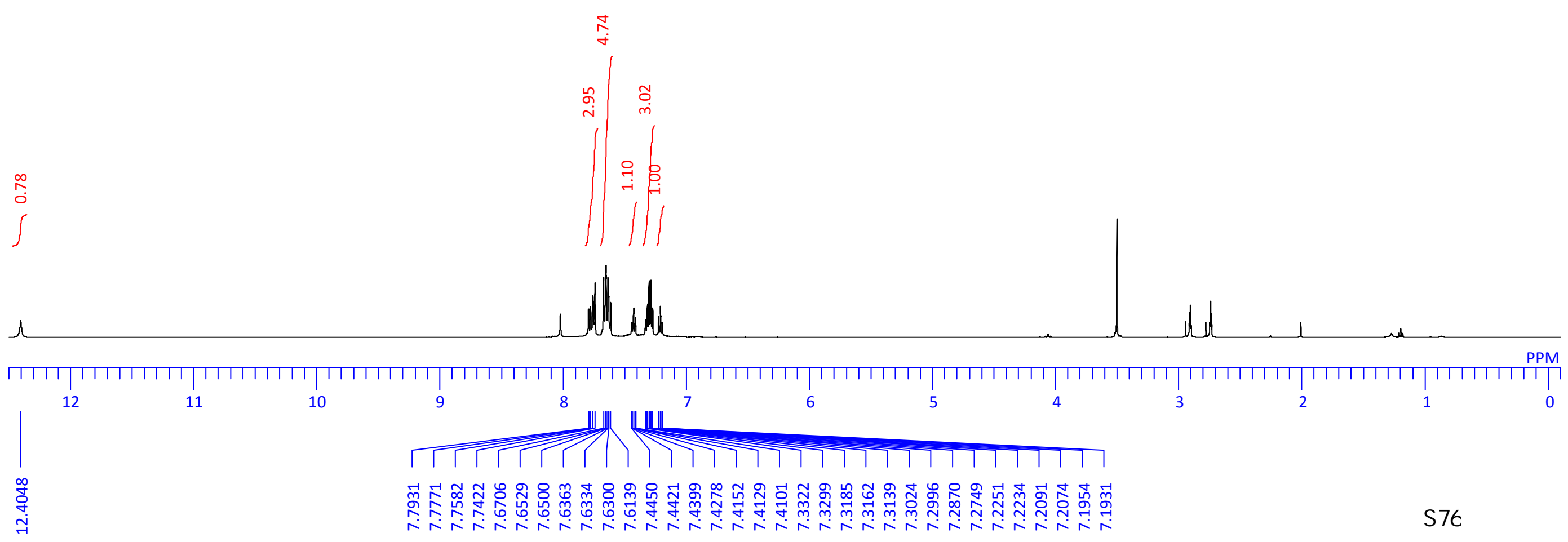


<smiles>O=C(c1ccccc1)c1c(-c2ccc(C(F)(F)F)cc2)[nH]c2ccccc12</smiles>

$1 e$

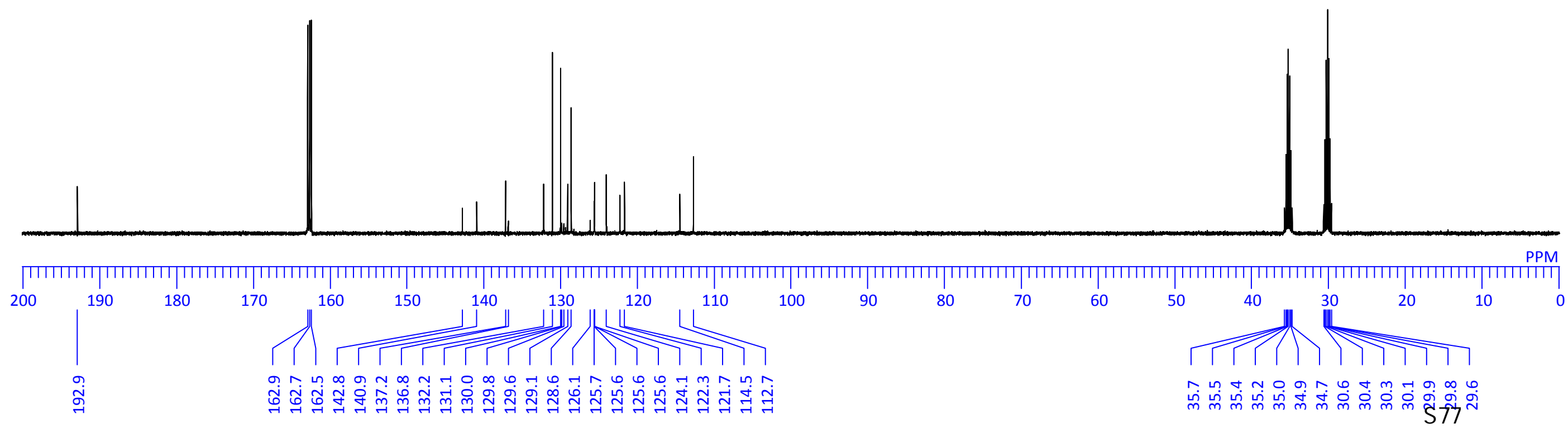




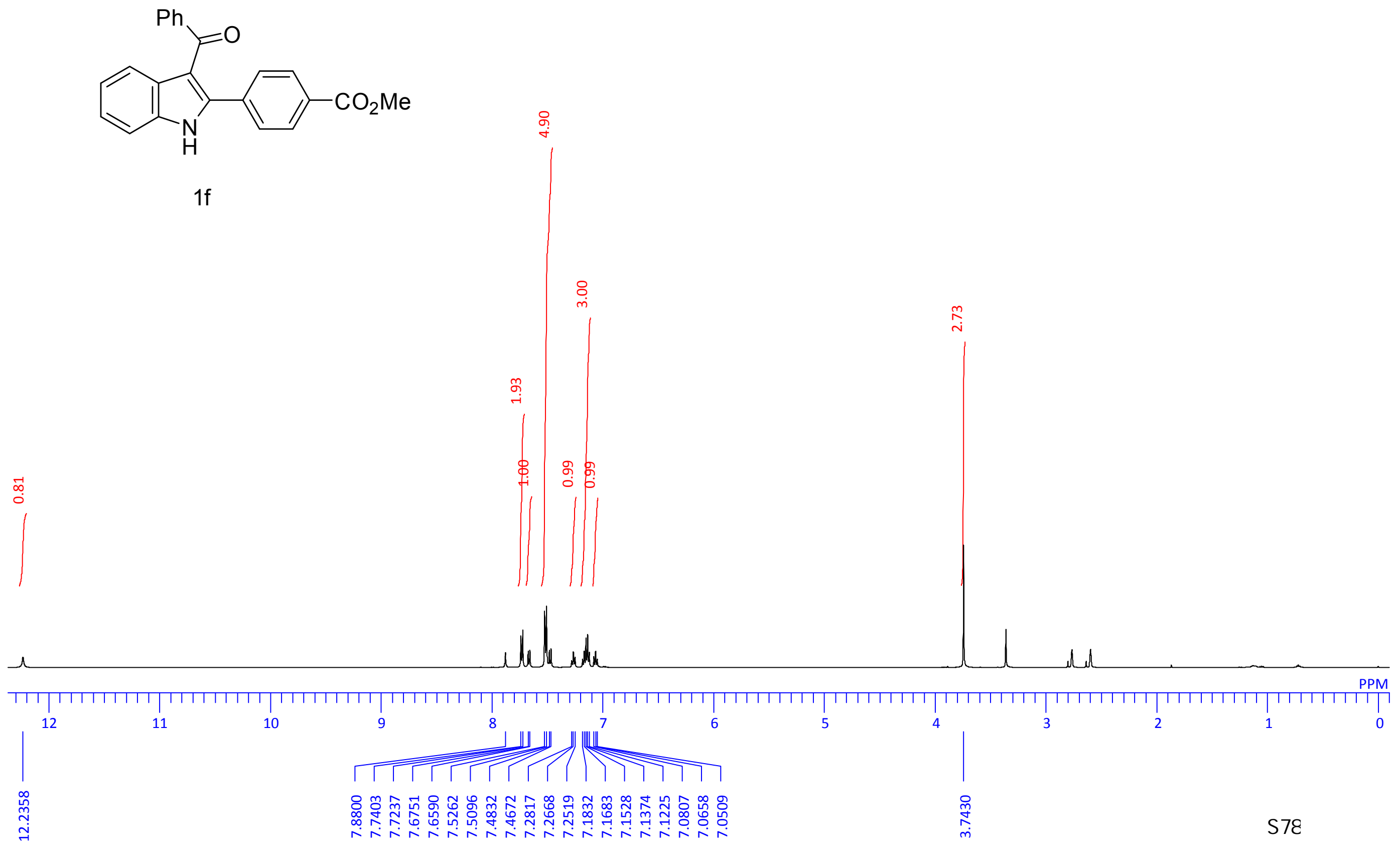




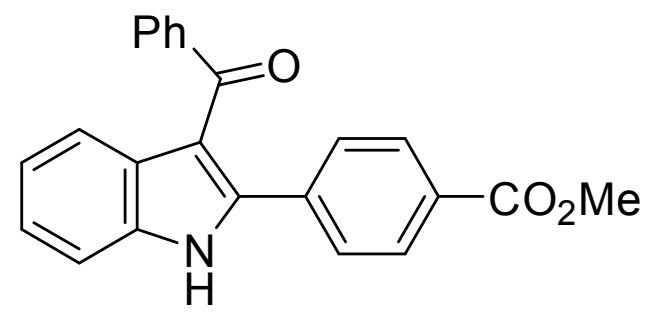

$1 f$

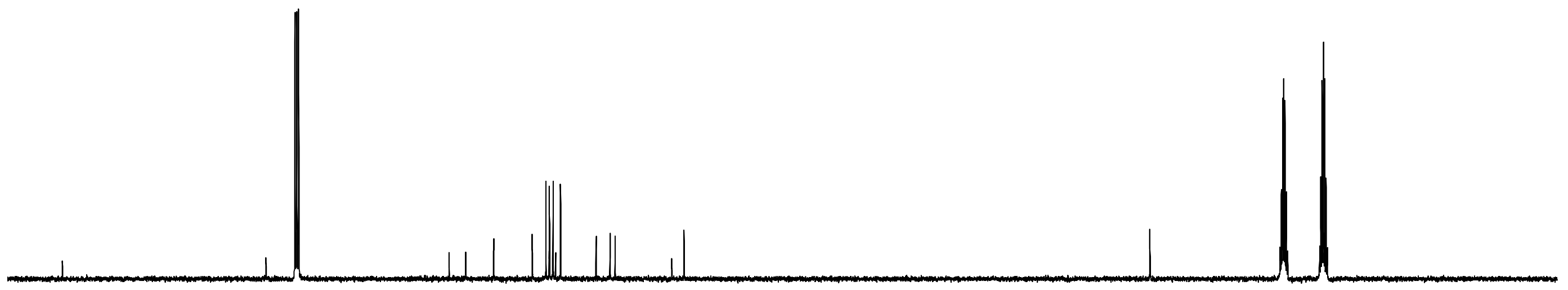
2

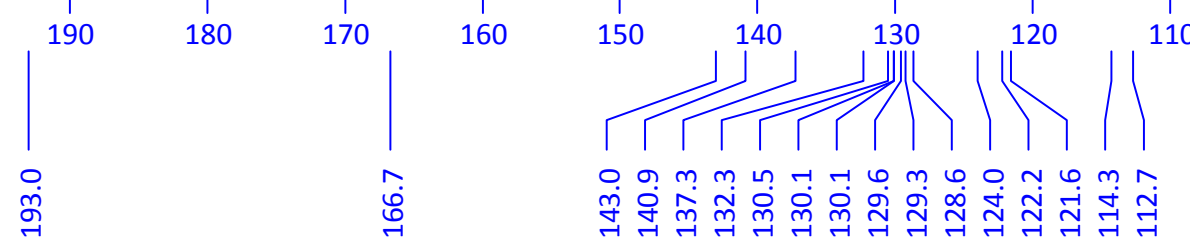

100

90

80

70

60

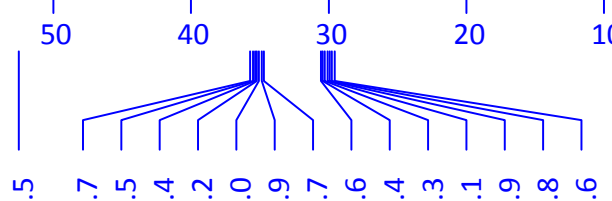

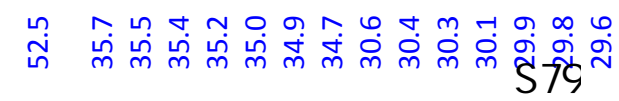


<smiles>N#Cc1ccc(-c2[nH]c3ccccc3c2C(=O)c2ccccc2)cc1</smiles>

$1 \mathrm{~g}$ $\stackrel{\circ}{\circ}$
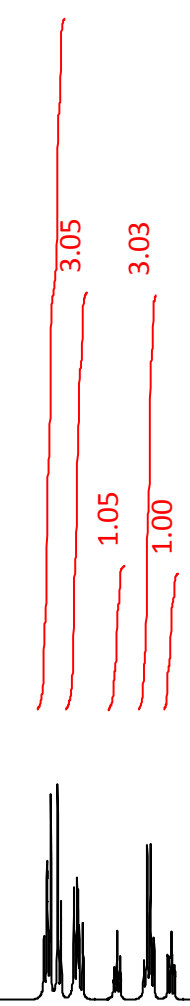

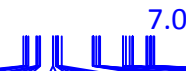

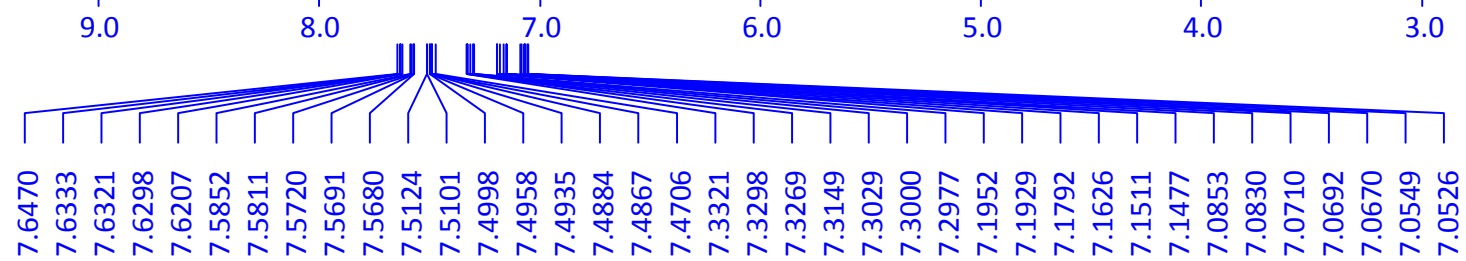

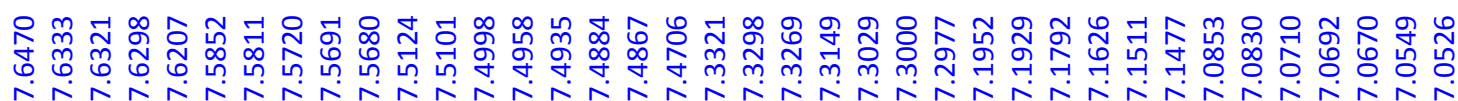




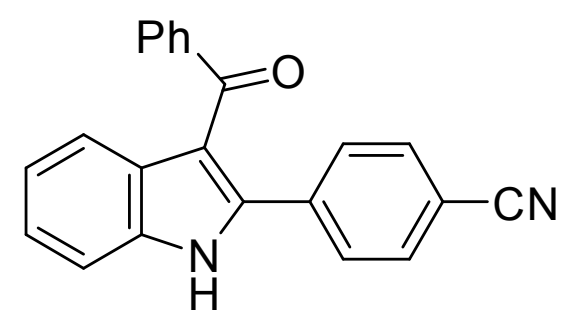

$1 \mathrm{~g}$
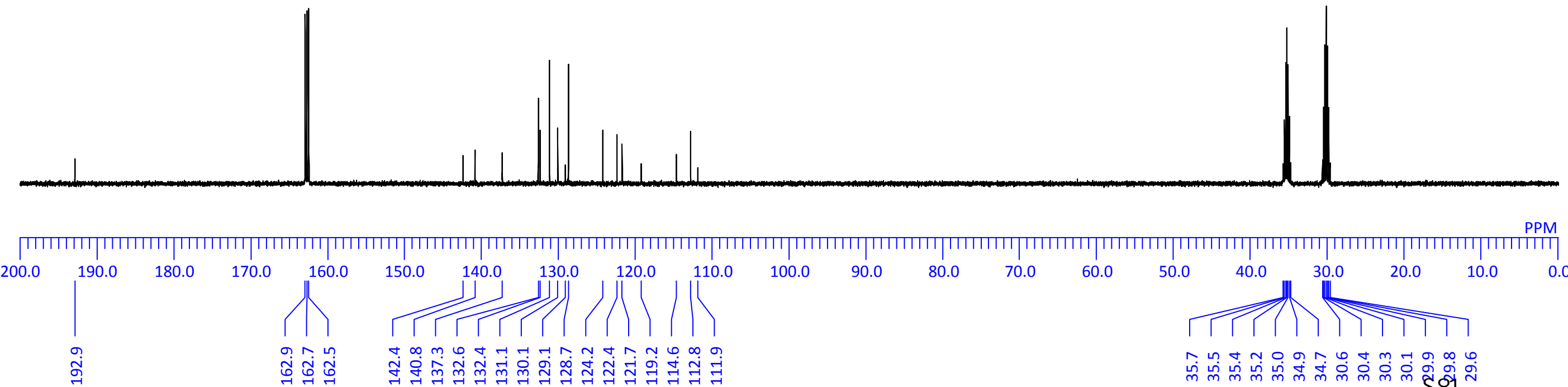

$\begin{array}{lll}100.0 & 90.0 \quad 80.0\end{array}$

$70.0 \quad 60.0$

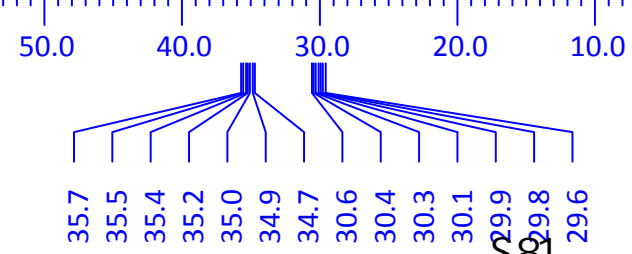

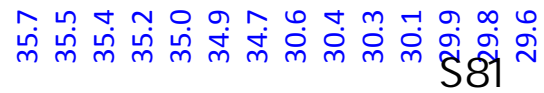




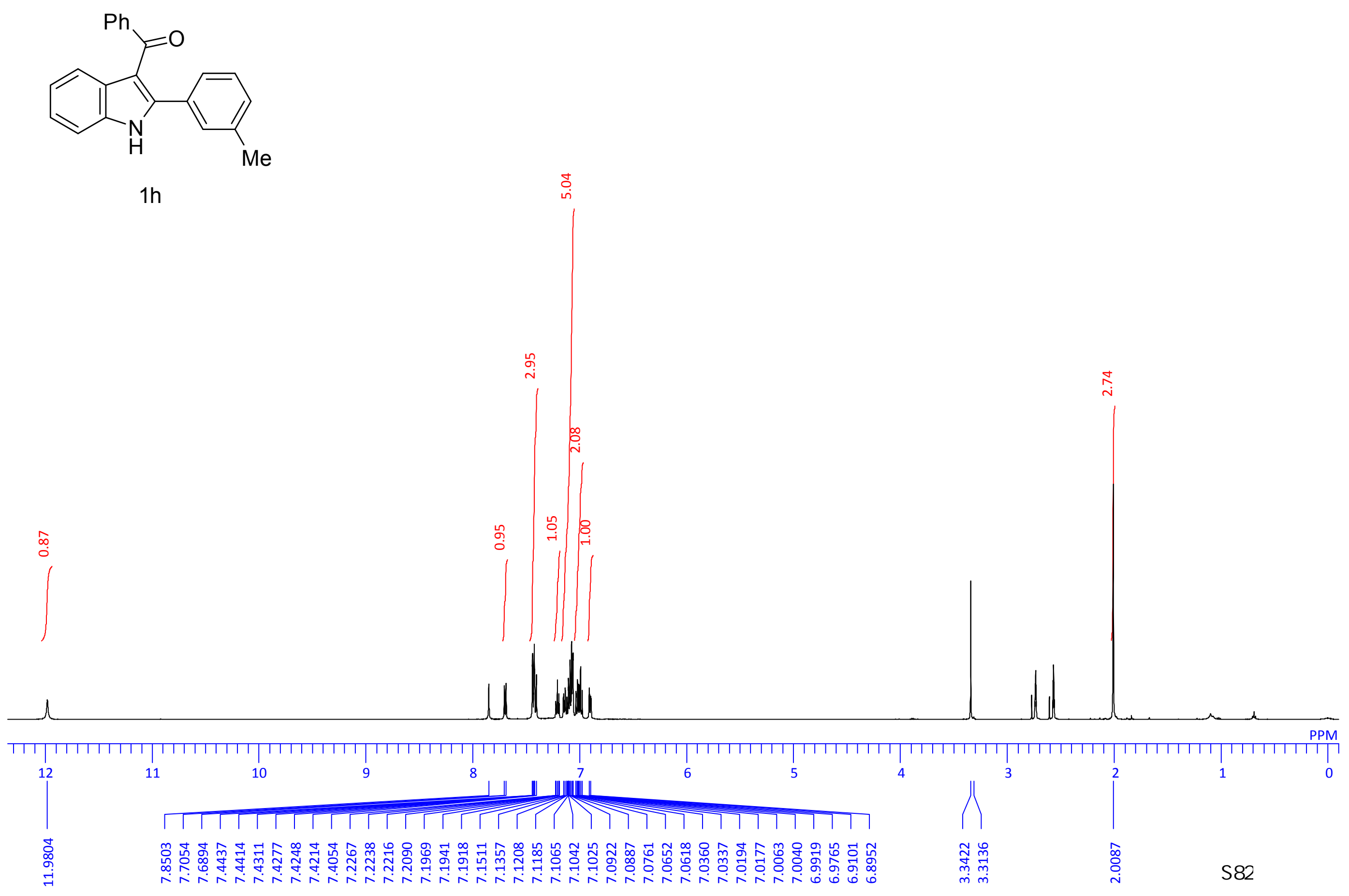




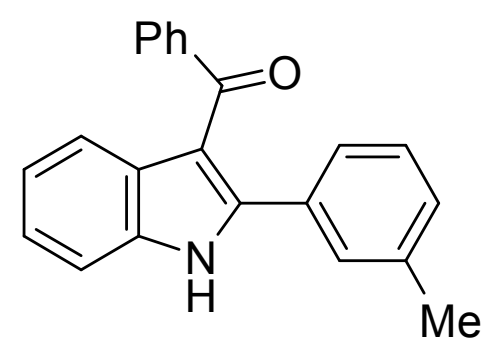

1h
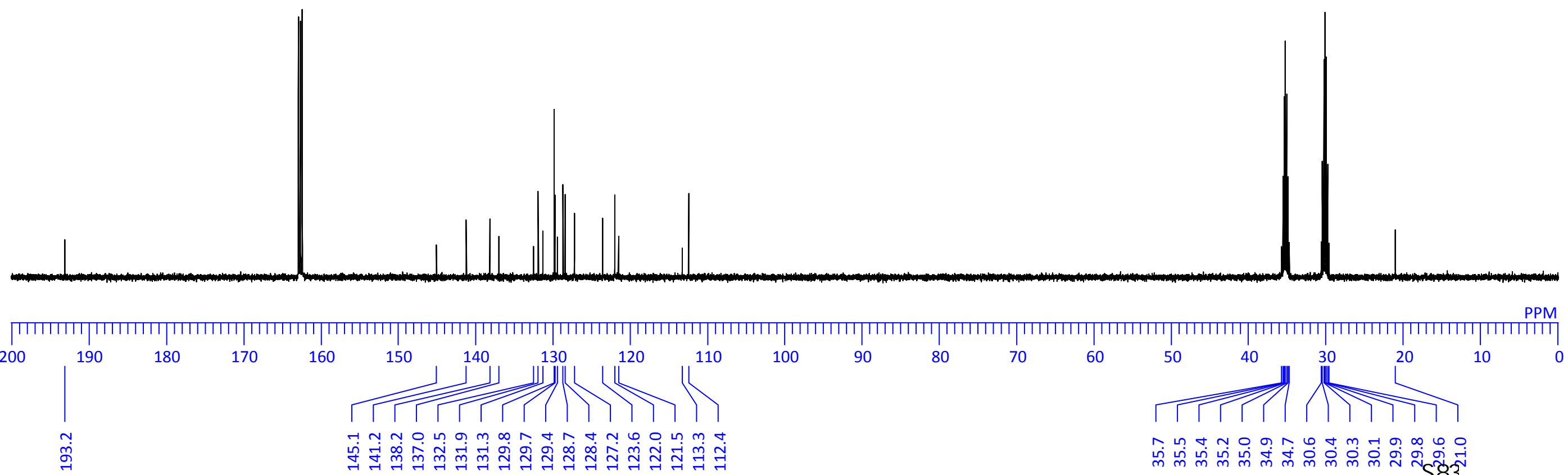

100

90

80

70

60

50
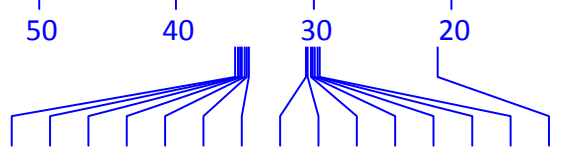

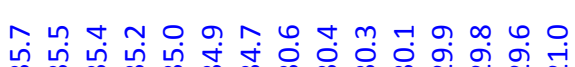

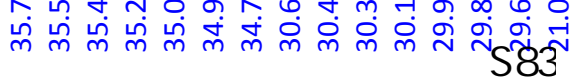




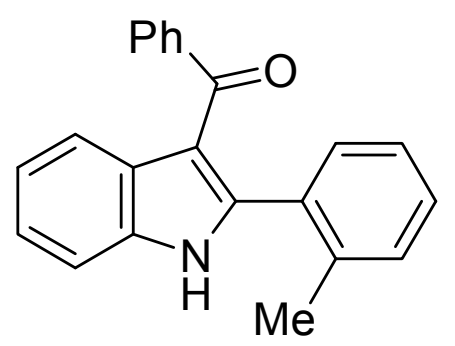

$1 \mathrm{i}$

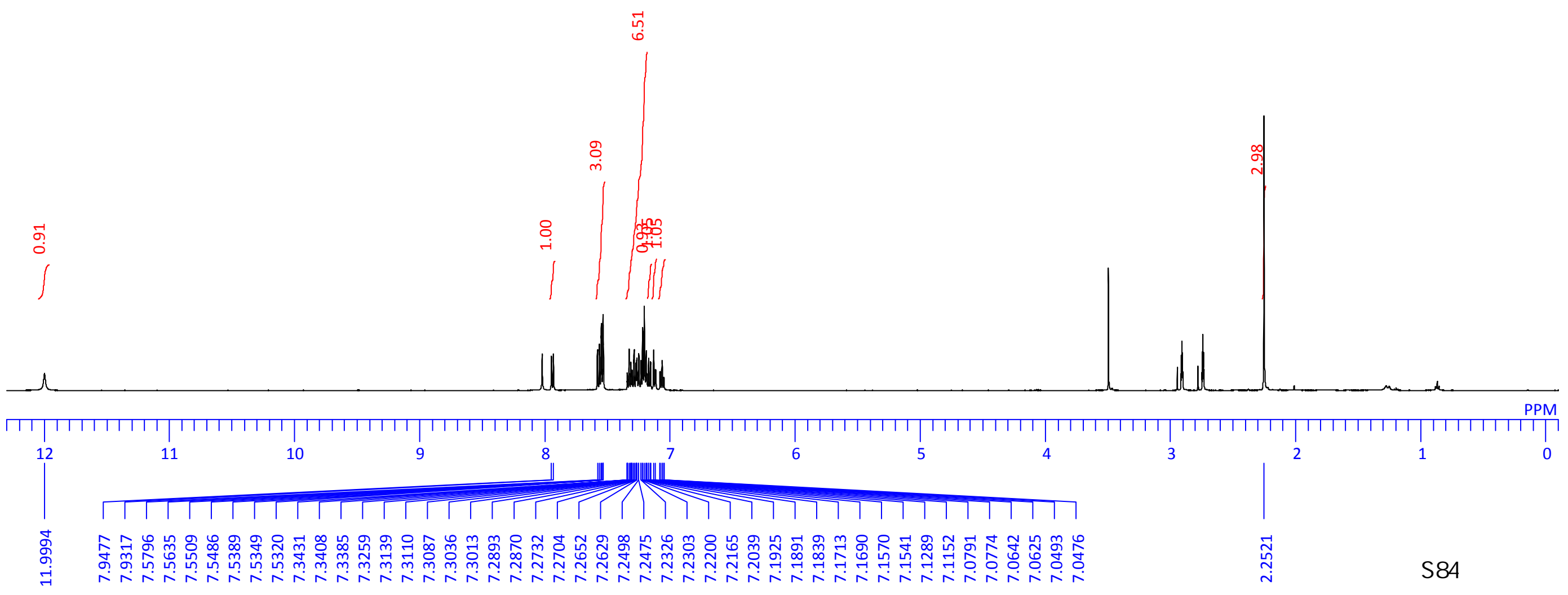




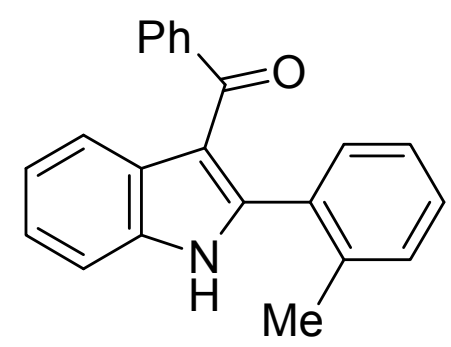

$1 \mathrm{i}$

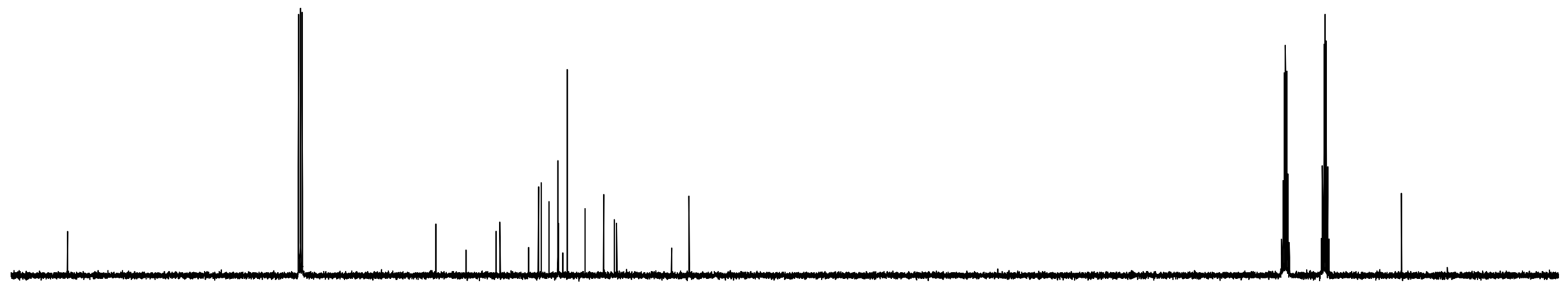
2

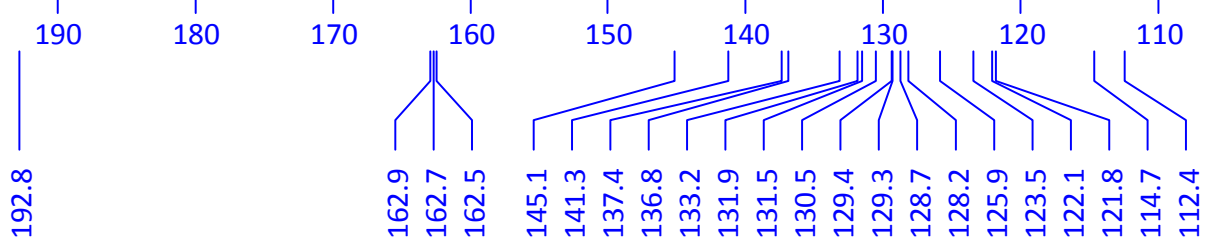

100

90

$80 \quad 70$

60

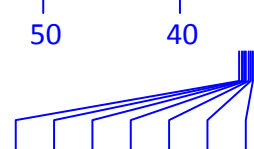

30

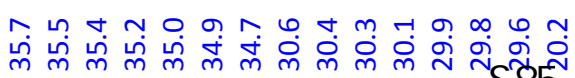


<smiles>O=C(c1ccccc1)c1c(-c2ccccc2)[nH]c2ccc(Cl)cc12</smiles>

1j

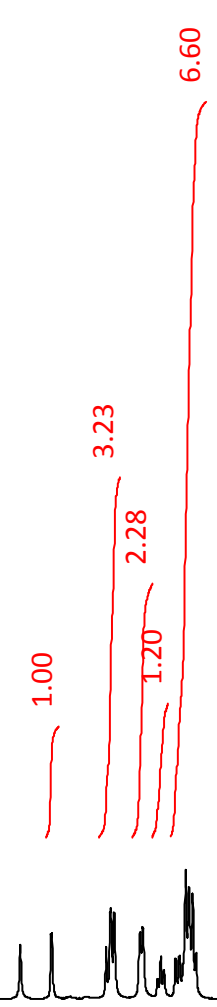

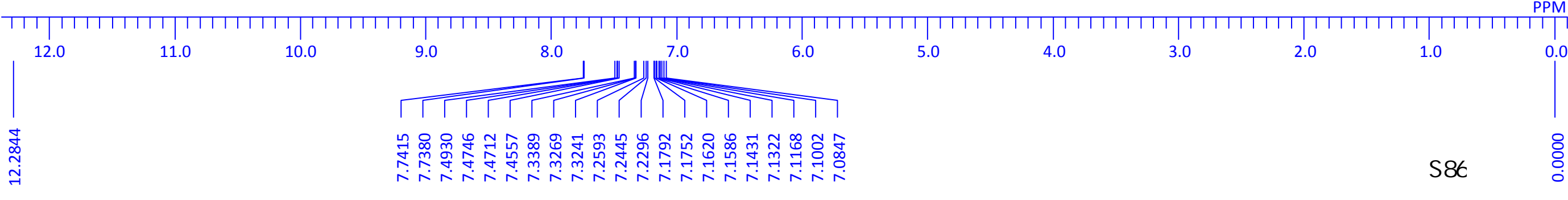


<smiles>O=C(c1ccccc1)c1c(-c2ccccc2)[nH]c2ccc(Cl)cc12</smiles>

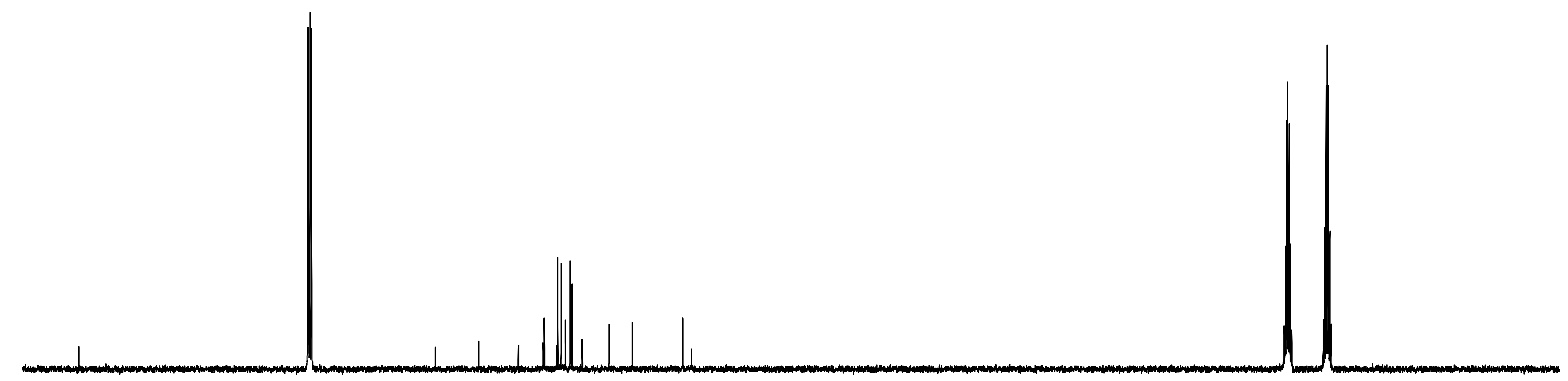

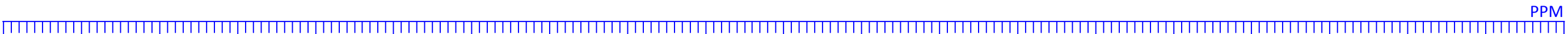
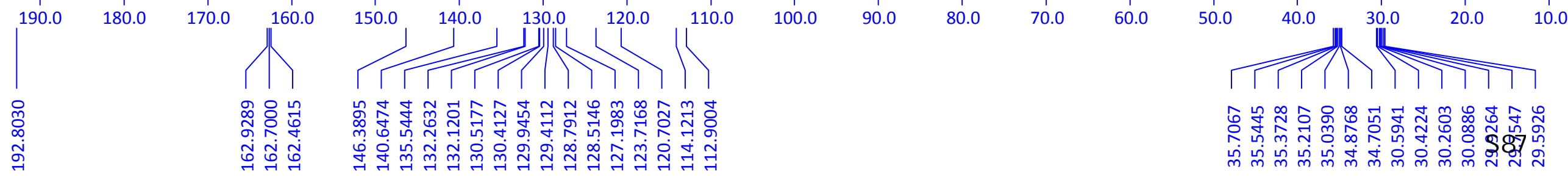


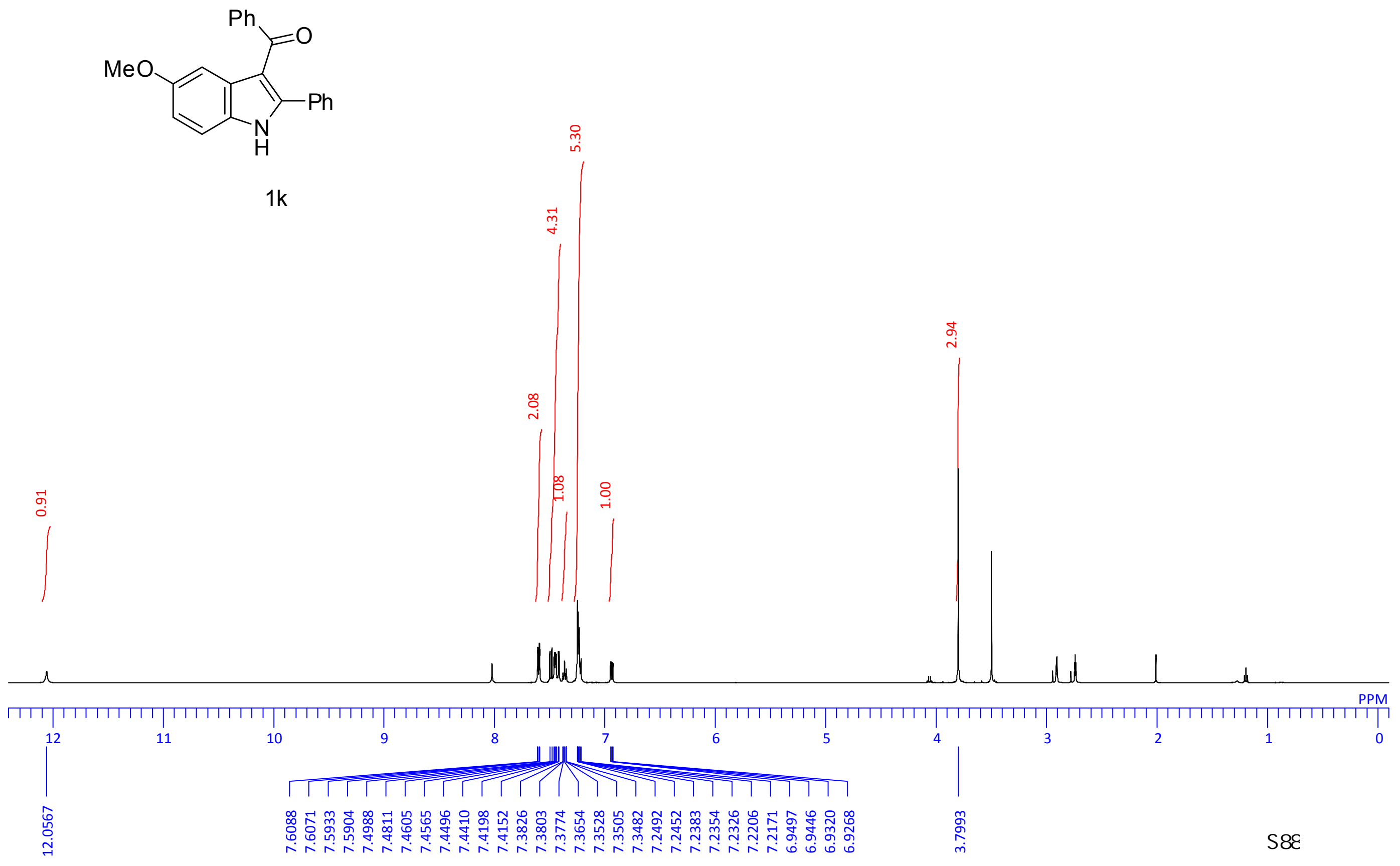


<smiles>COc1ccc2[nH]c(-c3ccccc3)c(C(=O)c3ccccc3)c2c1</smiles>

$1 \mathrm{k}$

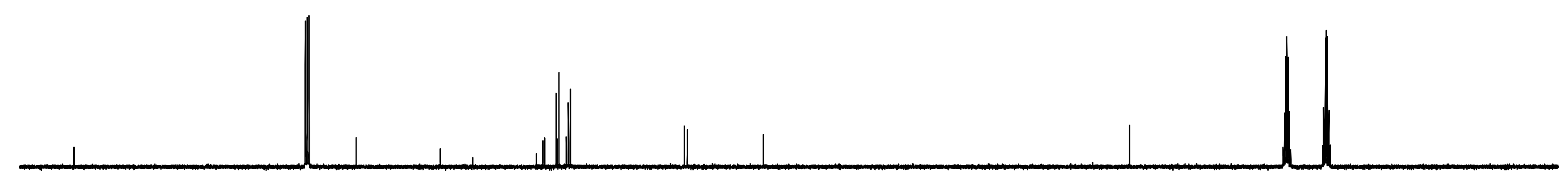

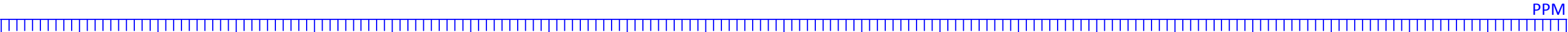
200

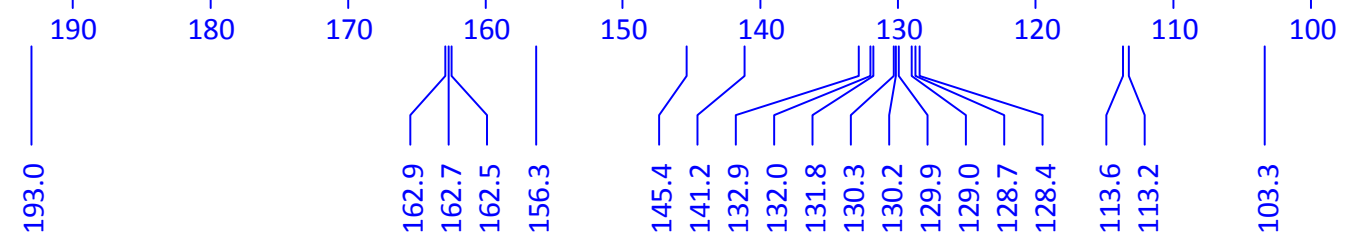

90

80
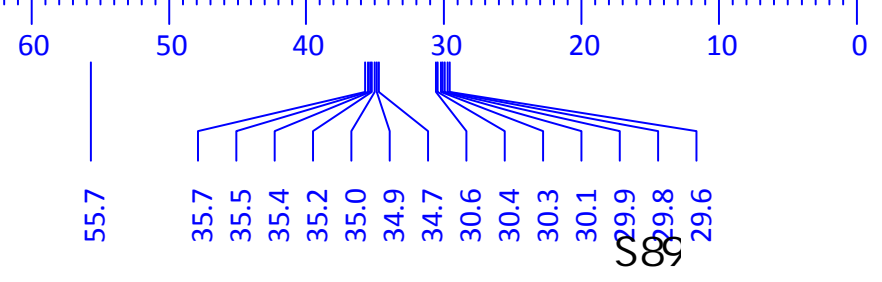


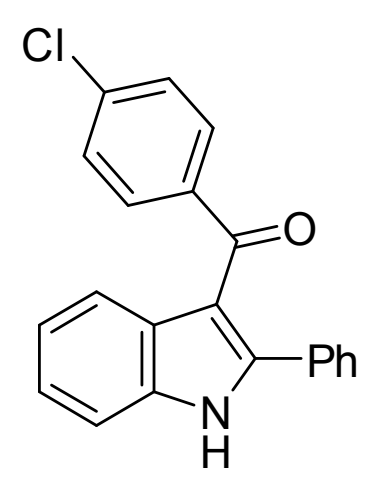

1

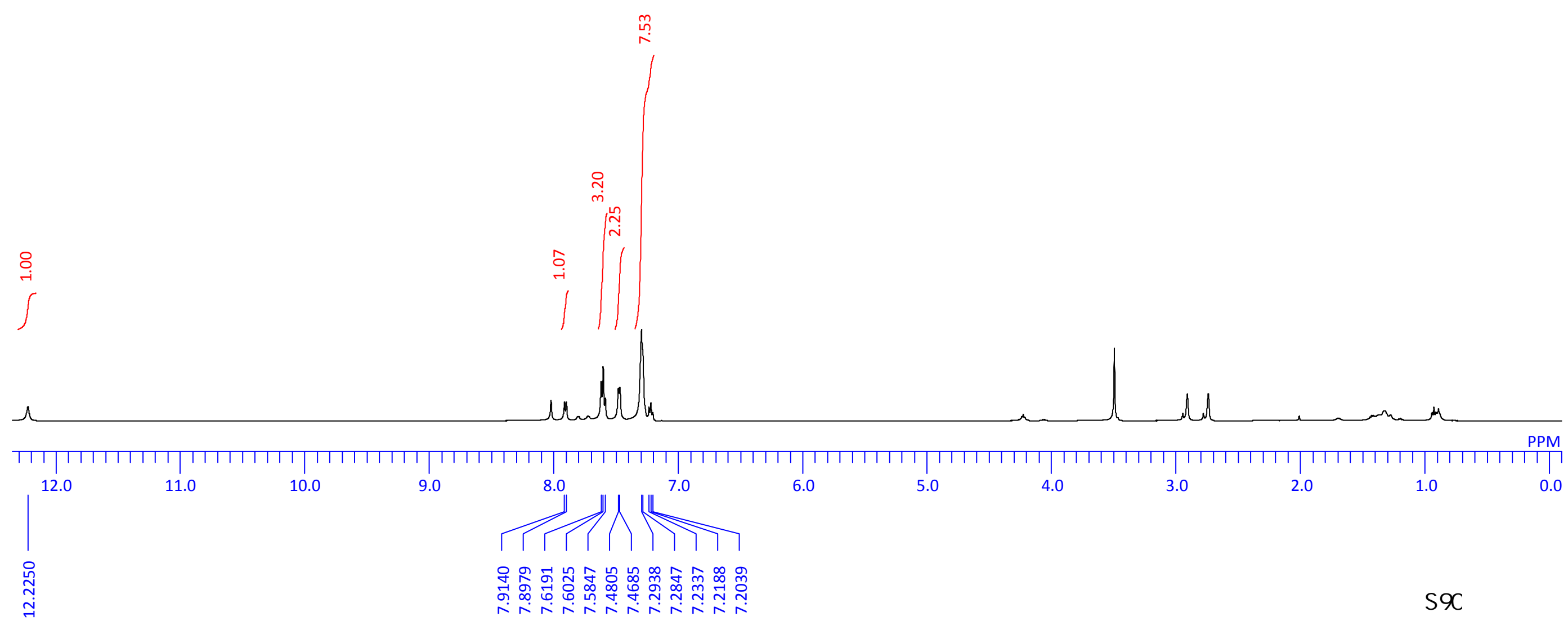



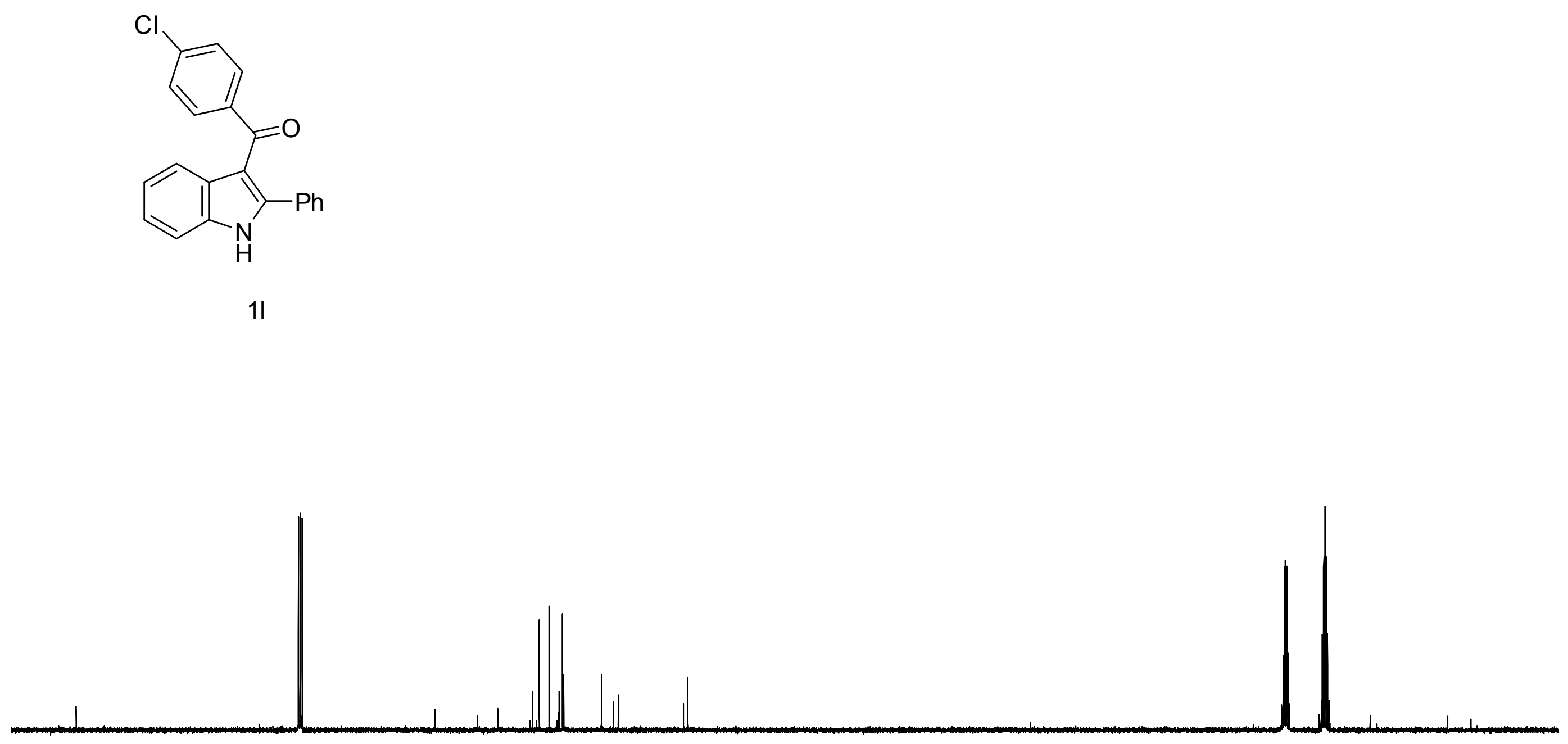

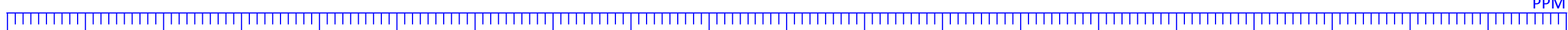

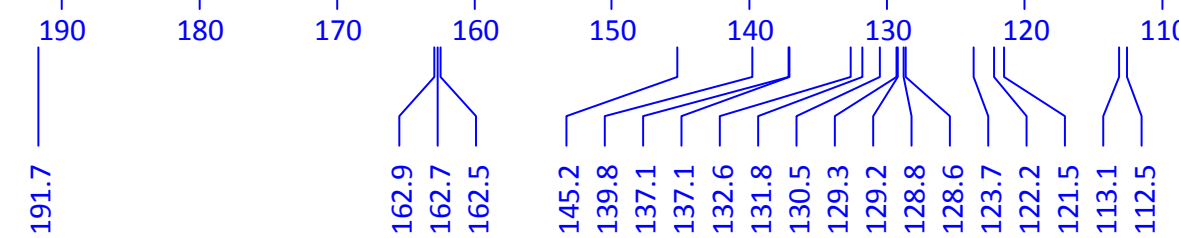

80

60

\section{0}

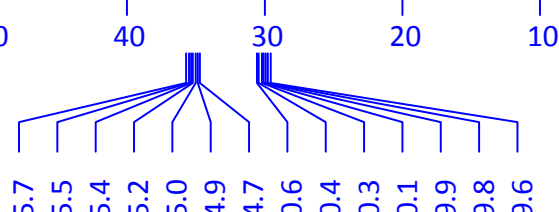

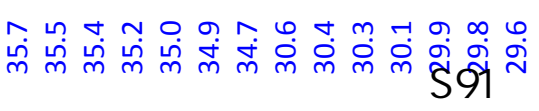




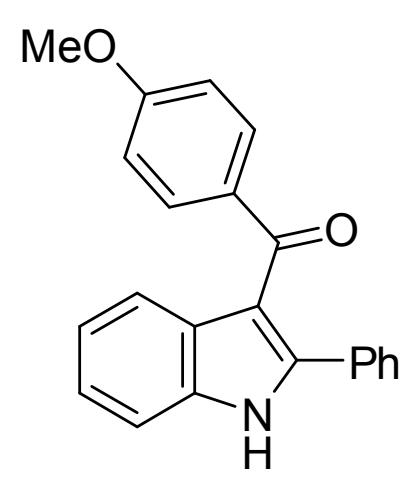

$1 \mathrm{~m}$

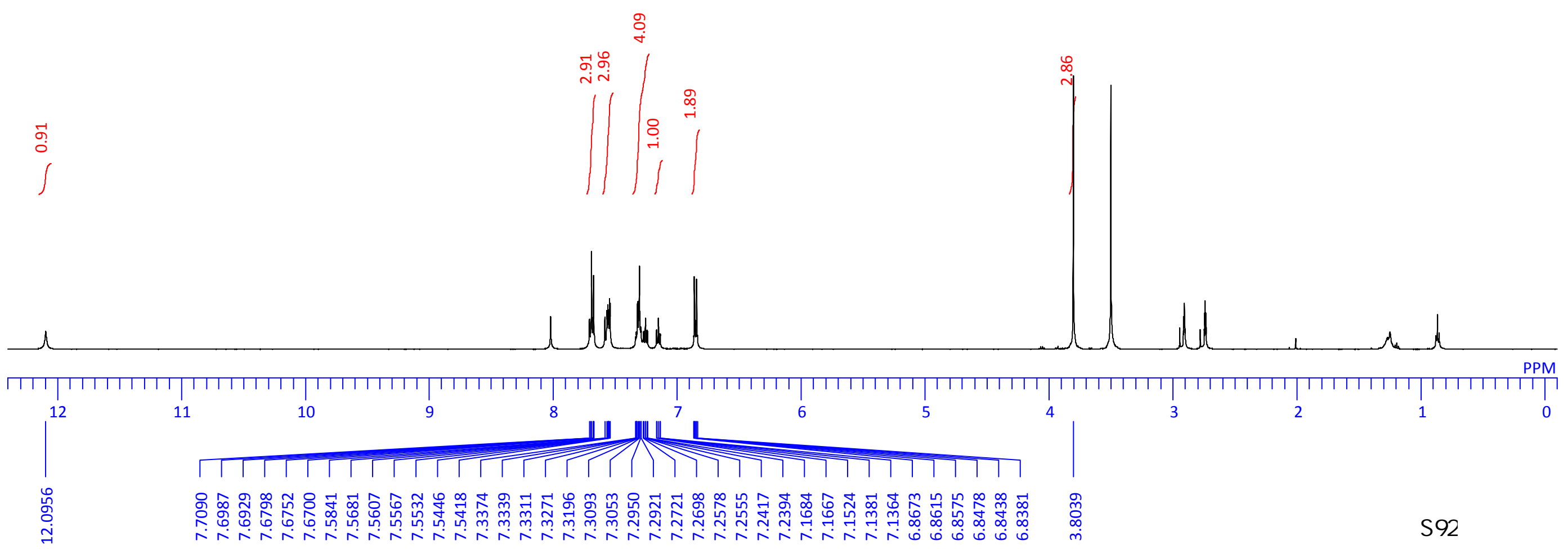




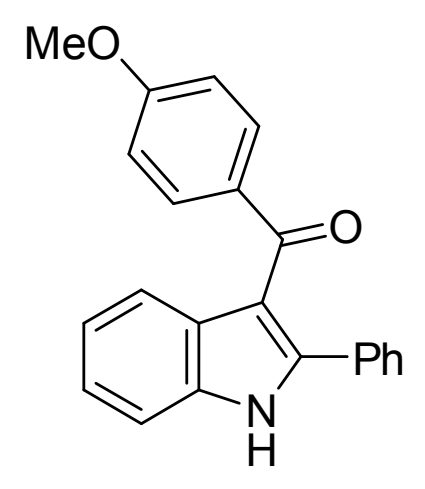

$1 \mathrm{~m}$

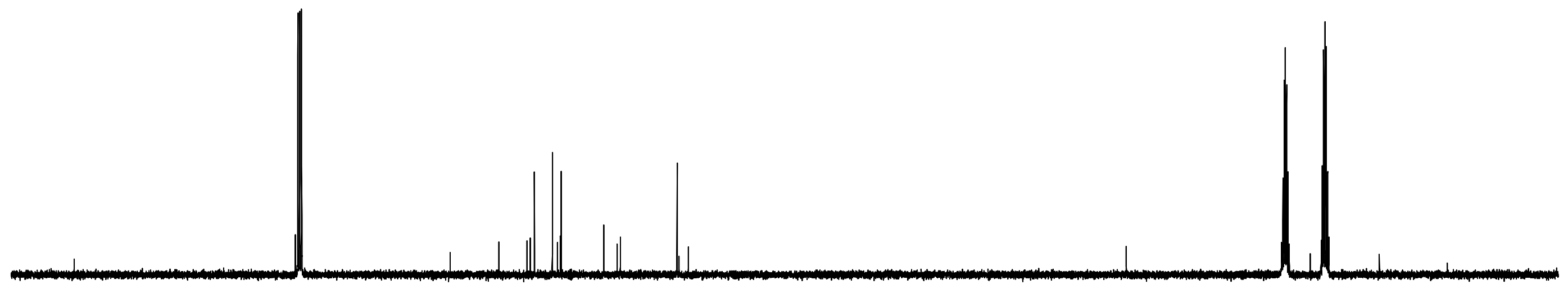
2

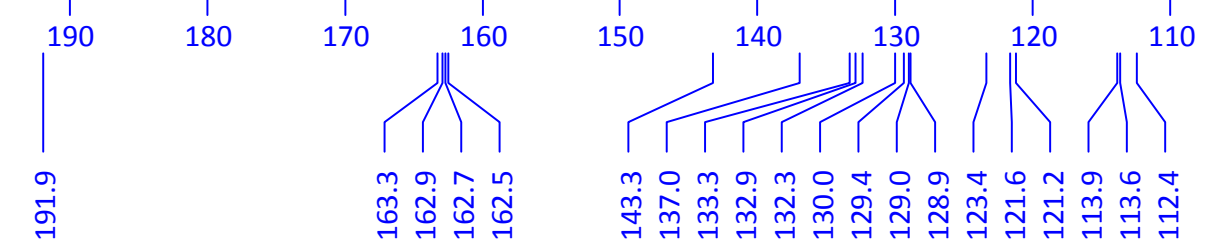

100

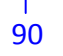

80

70

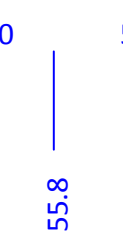

50

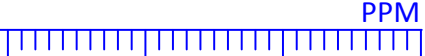



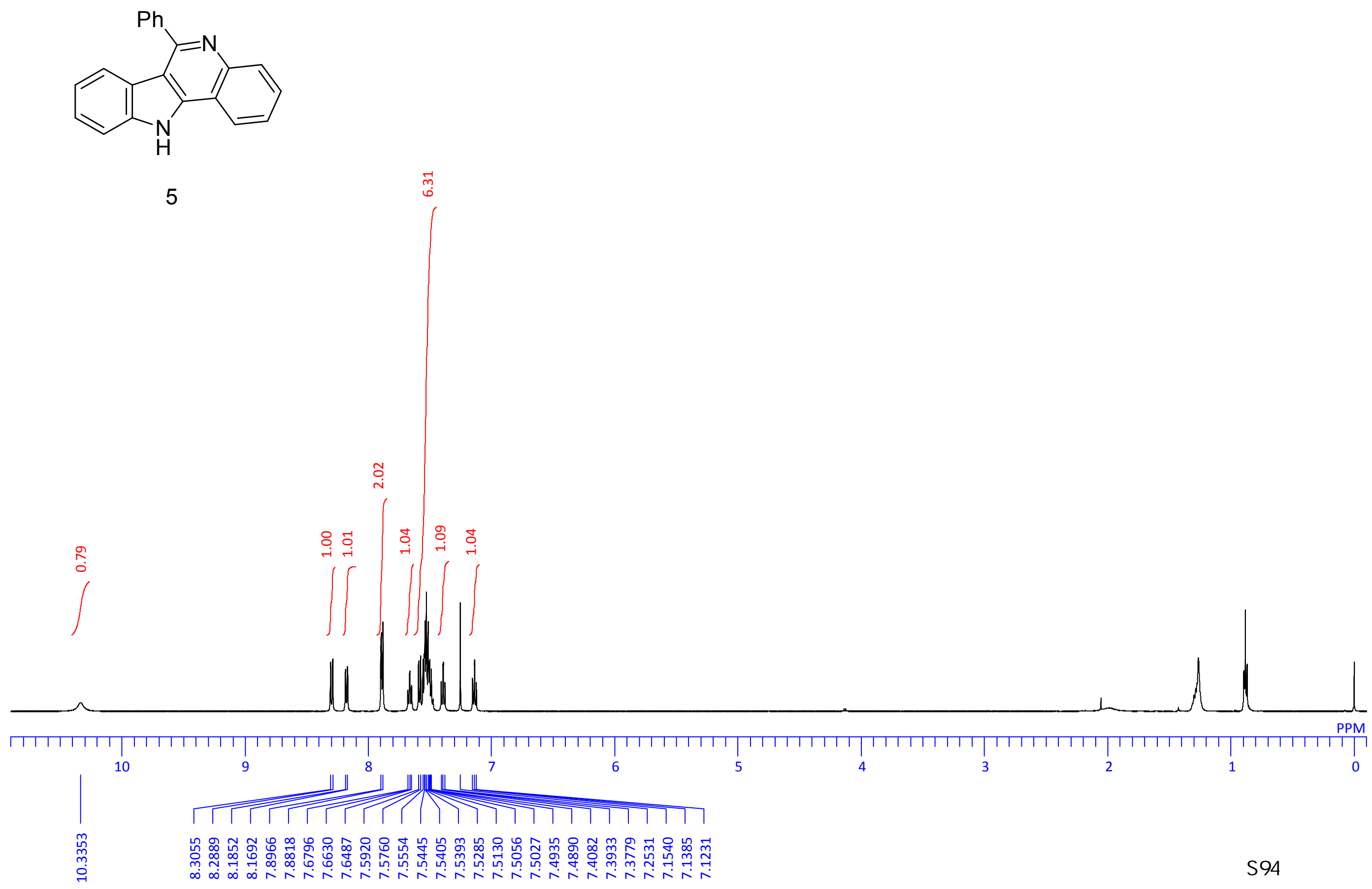


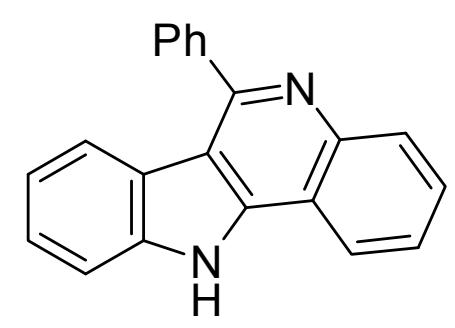

5

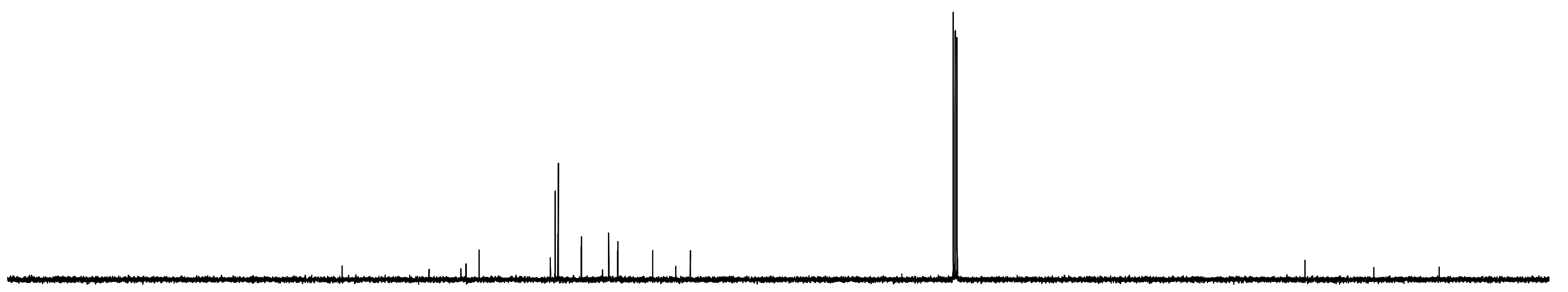

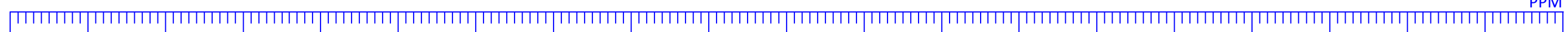
190

180

$170 \quad 160$

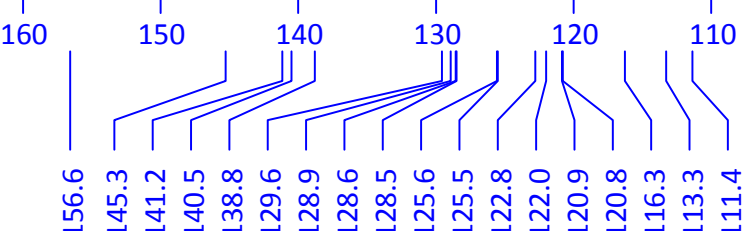

100

90

$\prod_{m \circ \infty}^{80}$

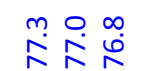




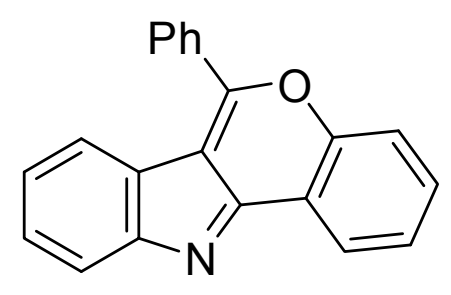

7

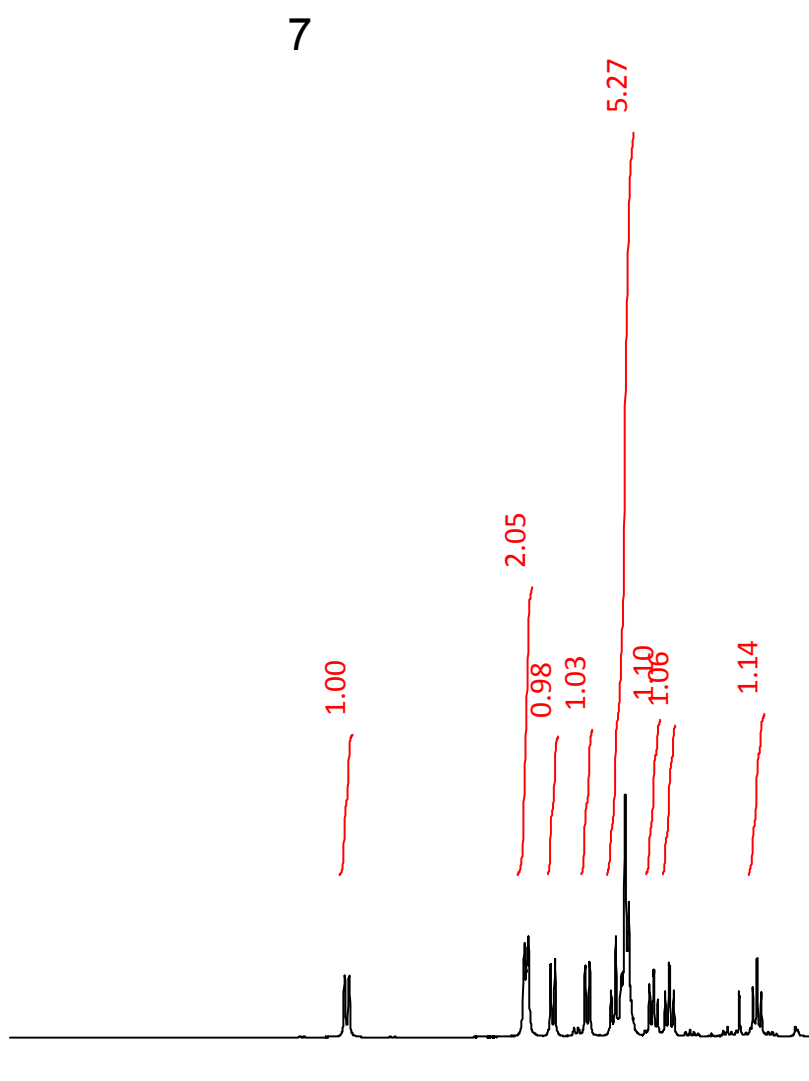

9

111 1 1 11110

1

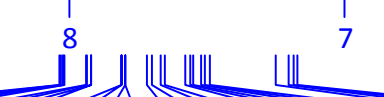

\begin{tabular}{l|l|l|l|}
11 & 1 & 1 & 1
\end{tabular}

6

$\prod_{5}$

4

3

2

2

(1)

1

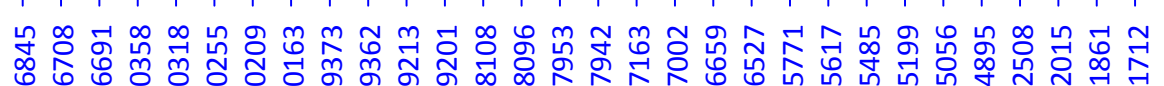

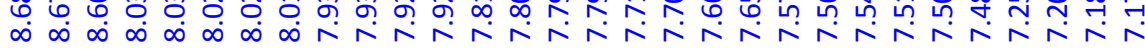




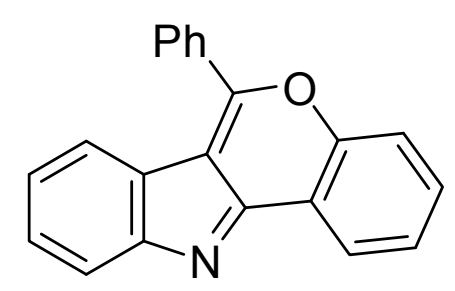

7

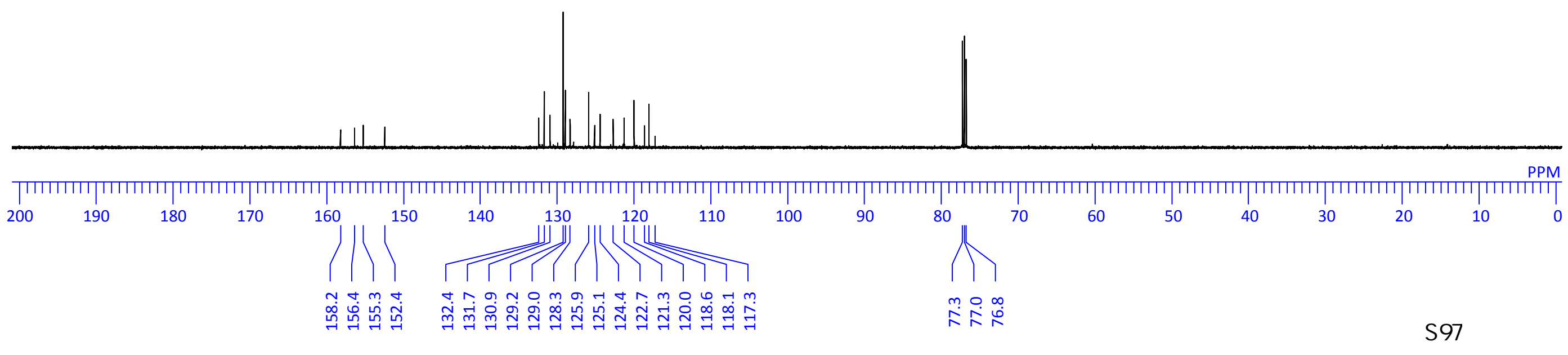



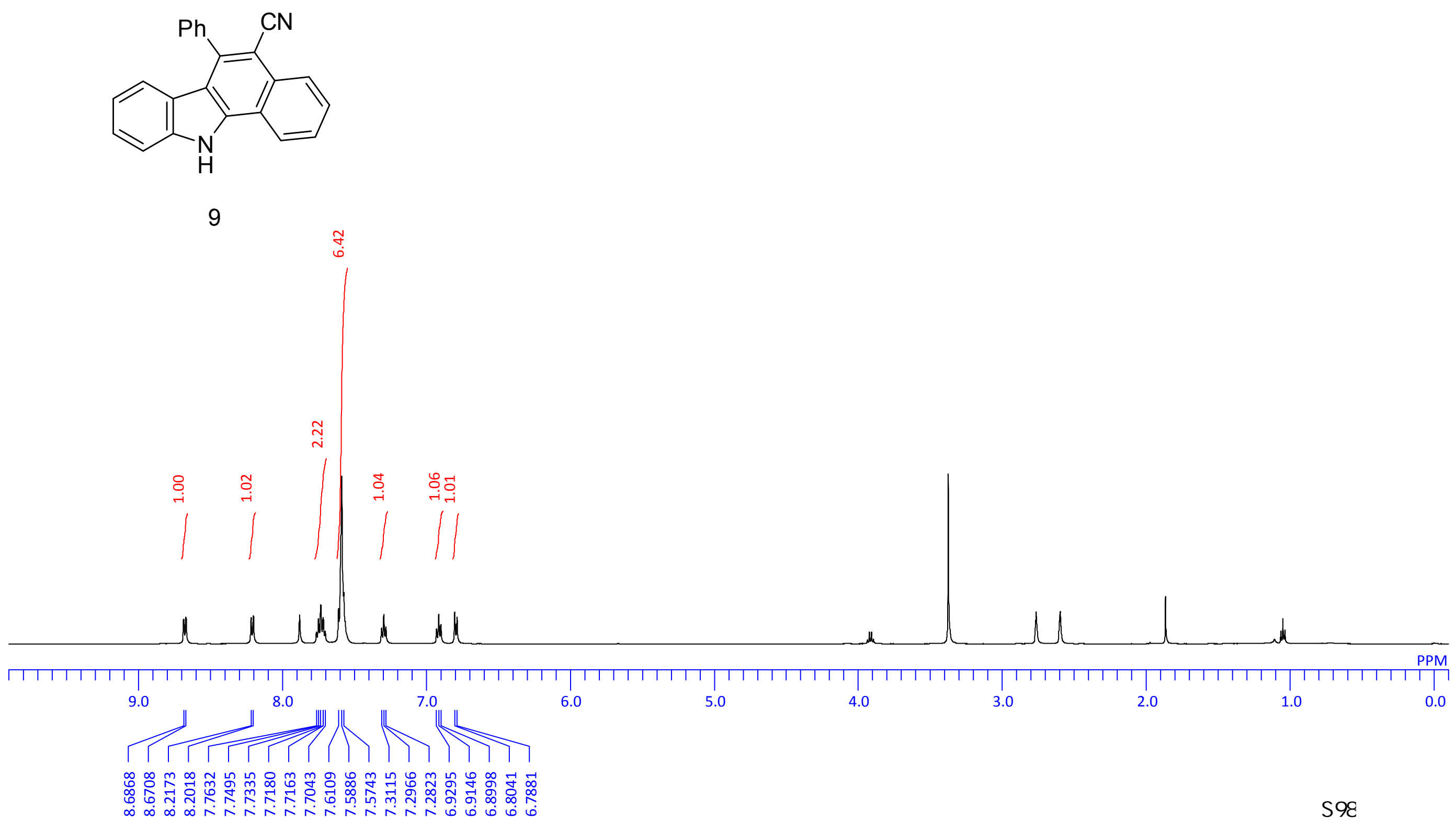
<smiles>N#Cc1c(-c2ccccc2)c2c3ccccc3[nH]c2c2ccccc12</smiles>

9
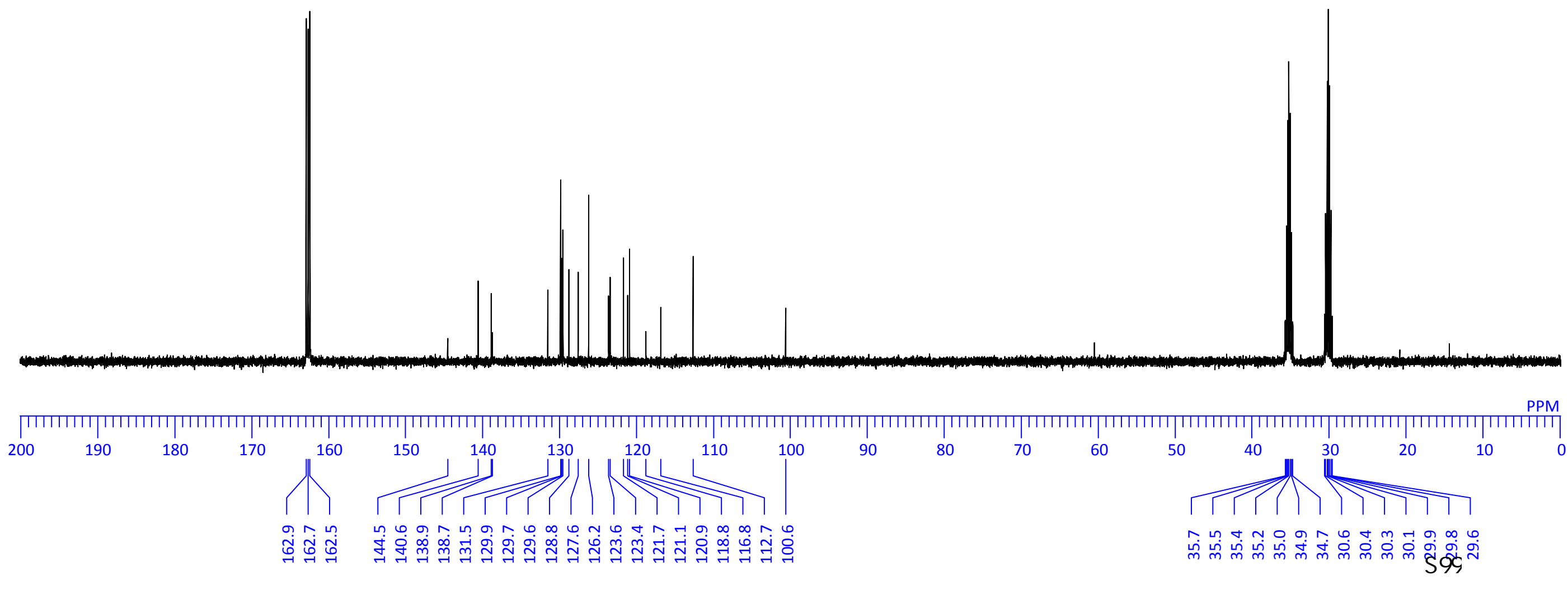